\title{
Symmetriebrechende Gitterverzerrung in einer elektronischen nematischen Phase
}

\section{Dissertation}

zur Erlangung des mathematisch-naturwissenschaftlichen Doktorgrades „Doctor rerum naturalium“

(Dr. rer. nat.)

der Georg-August-Universität Göttingen

von Christian Matthias Stingl

aus Lemgo

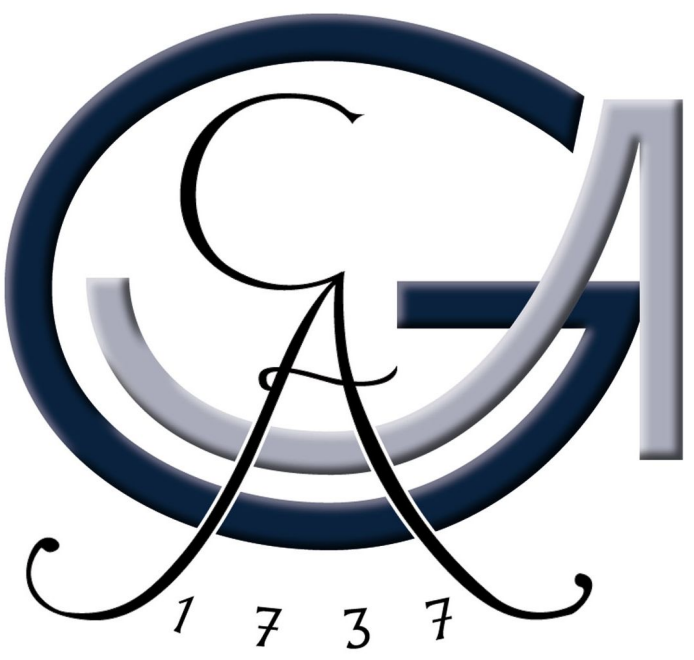

Göttingen 2011 
Referent: Prof. Dr. Philipp Gegenwart

Korreferent: Prof. Dr. Michael Lang

Tag der mündlichen Prüfung: 31. Mai 2011 
"Solid-state physics [...] worries too much about special substances."

— The Feynman Lectures on Physics, Vol. II 



\section{Inhaltsverzeichnis}

$\begin{array}{lll}1 & \text { Einleitung } & 7\end{array}$

2 Theoretische Grundlagen 11

2.1 Fermiflüssigkeits-Theorie . . . . . . . . . . . . . . . . . . . . . 11

2.2 Klassische Phasenübergänge . . . . . . . . . . . . . . . . . . . 13

2.3 Quantenphasenübergänge . . . . . . . . . . . . . . . . . . . . . 17

2.4 Thermodynamische Meßgrößen . . . . . . . . . . . . . . . . . . 20

3 Experimentelle Methoden und Datenanalyse 25

3.1 Erzeugung tiefer Temperaturen $\ldots \ldots \ldots \ldots \ldots \ldots \ldots$

3.2 Dilatometrie. . . . . . . . . . . . . . . . . . . . . . 25

3.2 .1 Prinzip und Aufbau . . . . . . . . . . . . . . . . . . 26

3.2 .2 Berechnung der Längenänderung . . . . . . . . . . . . . . 28

3.2 .3 Funktionstest . . . . . . . . . . . . . . . . . . . . . . . . . . . . . . 29

3.2 .4 Druck auf die Probe . . . . . . . . . . . . . . . . . . 30

3.2 .5 Messung des Hintergrunds . . . . . . . . . . . . . . . . . . 31

3.3 Numerische Differentiation $\ldots \ldots \ldots \ldots$. . . . . . . . . . . . . 32

4 Der itinerante Metamagnet $\mathrm{Sr}_{3} \mathrm{Ru}_{2} \mathrm{O}_{7}$

4.1 Kristallstruktur und elektronische Eigenschaften . . . . . . . . . . 37

4.2 Metamagnetismus und Quantenkritikalität . . . . . . . . . . . . . . . 39

4.3 Magnetoelastische Kopplung und thermische Ausdehnung . . . . . . . . 42

4.4 Nematische Phase $\ldots \ldots \ldots \ldots \ldots \ldots \ldots \ldots \ldots \ldots$

4.4 .1 Domänenmodell . . . . . . . . . . . . . . . . . 45

4.5 Spezifische Wärme und Entropie . . . . . . . . . . . . . . . 46

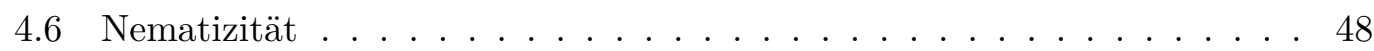

4.6 .1 Theorien für $\mathrm{Sr}_{3} \mathrm{Ru}_{2} \mathrm{O}_{7} \ldots \ldots \ldots \ldots \ldots \ldots \ldots \ldots$ 
$\begin{array}{lll}5 & \text { Messungen und Ergebnisse } & \mathbf{5 1}\end{array}$

$5.1 \quad$ Dilatometrie an $\mathrm{Sr}_{3} \mathrm{Ru}_{2} \mathrm{O}_{7} \ldots \ldots \ldots \ldots \ldots \ldots \ldots$

$5.1 .1 \quad$ Unverkippte Konfiguration . . . . . . . . . . . . . . . 52

5.1 .2 Verkippte Felder . . . . . . . . . . . . . . . . . . . 58

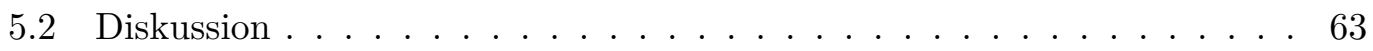

5.2 .1 Verzerrung $\ldots \ldots \ldots \ldots \ldots \ldots$

5.2 .2 Quantenkritischer Einfluß . . . . . . . . . . . . . . . 68

$5.2 .3 \quad$ Nematischer Phasenübergang . . . . . . . . . . . . . . . . . 70

5.3 Entropie . . . . . . . . . . . . . . . . . . . . . 75

5.4 Spezifische Wärme . . . . . . . . . . . . . . . . . . 77

\begin{tabular}{lll}
\hline 6 & Zusammenfassung & 81
\end{tabular}

\begin{tabular}{lr}
\hline Literatur & 85
\end{tabular} 


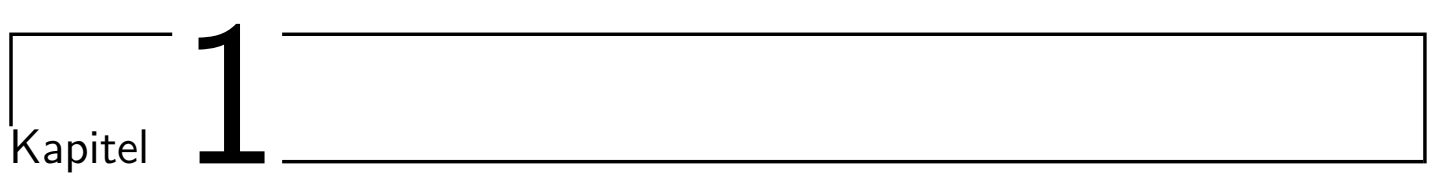

\section{Einleitung}

Die Festkörperphysik gehört sicherlich zu den physikalischen Disziplinen, die in den letzten Jahrzehnten die weitestreichenden technischen und gesellschaftlichen Auswirkungen entwickelt haben. Die elektronischen Eigenschaften von anwendungsrelevanten Materialien wie Metallen und Halbleitern sind im Rahmen von quantenmechanischen Beschreibungen gut verstanden und seit Shockley, Bardeen und Brattain 1947 den ersten Bipolartransistor vorstellten, hat die Elektronik rasante Fortschritte gemacht. Neue Entwicklungen sowohl in der Forschung als auch in den Produktionsprozessen treiben eine stetige Miniaturisierung und Leistungssteigerung, deren Ende nach wie vor nicht abzusehen ist. Die heute allgegenwärtige Verfügbarkeit von Informationsund Kommunikationstechnik, die in ihren gesellschaftlichen Auswirkungen einer zweiten industriellen Revolution gleichkommt, ist damit letztlich auch ein Erfolg der festkörperphysikalischen Grundlagenforschung.

Aber auch abseits der gut verstandenen Bereiche gibt es in der Festkörperphysik noch Neues zu entdecken: so wurden gerade bei tiefen Temperaturen immer wieder ungewöhnliche Zustände von kondensierter Materie gefunden. Das prominenteste Beispiel ist hier sicherlich die Supraleitung, die KAMERLINGH-OnNES 1911 entdeckte [KO11, nachdem ihm 1908 die Verflüssigung von Helium gelungen war. Der Mechanismus hinter diesem erstaunlichen Effekt blieb lange unverstanden und es dauerte fast 50 Jahre, bis Bardeen, Cooper und Schrieffer 1957 ihre mikroskopische Theorie der Supraleitung vorlegen konnten BCS57.

Weitere Impulse erhielt das Gebiet mit der Entdeckung von unkonventioneller Supraleitung in starkkorrelierten Elektronensystemen. Während nämlich die Elektronen vieler „einfacher“ Metalle im Einteilchenbild beschrieben werden können, was bei etwa $10^{23}$ über die Coulombabstoßung wechselwirkenden Teilchen erstaunlich ist, können die Wechselwirkungen bei starken elektronischen Korrelationen nicht mehr vernachlässigt werden. Oft lassen sie sich im Rahmen von LANDAUs Theorie der Fermiflüssigkeit behandeln und die kollektiven Anregungen als Quasiteilchen mit renormierter Masse beschreiben, doch auch diese außerordentlich erfolgreiche Theorie bricht bei neuar- 
tigem Verhalten zusammen, das sich in Ermangelung besserer Begriffe nur noch als Nicht-Fermiflüssigkeits-Verhalten bezeichnen läßt.

Unkonventionelle Supraleitung wurde 1979 in den Schwere-Fermionen-Systemen von Steglich [Ste79] und 1986 in den Kupferoxid-basierten Hochtemperatursupraleitern durch Bednorz und Müller BM86 gefunden. Beide Materialklassen wurden seitdem intensiv untersucht und die höchste bekannte Sprungtemperatur der Hochtemperatursupraleiter liegt mittlerweile bei $135 \mathrm{~K}$ (unter Druck werden sogar bis $160 \mathrm{~K}$ erreicht), doch ist die genaue Ursache für die Cooperpaarung in beiden Fällen bis heute unbekannt. Sie steht aber möglicherweise im Zusammenhang mit der Existenz eines Quantenkritischen Punktes (QKP). Ein solcher entsteht, wenn die Übergangstemperatur eines klassischen (magnetischen) Phasenübergangs durch einen externen Kontrollparameter zum absoluten Nullpunkt der Temperatur unterdrückt wird [GSS08]. Das System wird dann durch quantenmechanische Fluktuationen zwischen der geordneten und der ungeordneten Phase charakterisiert. Die Kopplung an solche Fluktuationen könnte möglicherweise die elektronische Paarbildung erklären [Bro08.

In den letzten zehn Jahren ist auch einem anderen neuartigen elektronischen Zustand große Aufmerksamkeit zuteil geworden: In elektronischen nematischen Phasen bricht das Elektronensystem spontan die zugrundeliegende Rotationssymmetrie des Kristallgitters. Bekannt sind nematische Phasen aus den Flüssigkristallen, in denen die einzelnen Moleküle eine stabförmige Geometrie haben und sich deshalb bevorzugt parallel anordnen, während der Freiheitsgrad der Translation wie in einer Flüssigkeit erhalten bleibt. Daß auch Elektronen als eigentlich punktförmige Teilchen nematische Eigenschaften zeigen können, ist dagegen äußerst überraschend. Es gibt aber in verschiedenen Systemen experimentelle Anhaltspunkte für ein solches Verhalten [Fra10].

Mit dem Material $\mathrm{Sr}_{3} \mathrm{Ru}_{2} \mathrm{O}_{7}$ existiert nun ein Modellsystem, welches sich durch gleich zwei der angesprochenen Phänomene auszeichnet. Einerseits findet man einen quantenkritischen Punkt, der aus der Unterdrückung eines metamagnetischen Übergangs hervorgeht. Durch eine starke magnetoelastische Kopplung äußern sich die quantenkritischen Fluktuationen in Signaturen der thermischen Ausdehnung, welche in hervorragendem Einklang mit der Standardtheorie der Quantenkritikalität stehen Geg06b. Andererseits bildet sich bei tiefen Temperaturen unter einem Grad Kelvin und hohen Magnetfeldern von etwa acht Tesla eine neuartige thermodynamische Phase mit anisotropen elektronischen Transporteigenschaften in unmittelbarer Nähe des QKPs aus Bor07. Diese als "nematisch“ bezeichnete Phase hat in den letzten Jahren ein großes Forschungsinteresse auf sich gezogen und ist besonders durch Widerstandsexperimente gut untersucht. Durch Transportmessungen in verschiedenen Richtungen läßt sich die Anisotropie zwar gut charakterisieren, doch stellt der Widerstand keine thermodynamische Meßgröße dar und ist außerdem abhängig von mikroskopischen Details wie einem konkreten Strompfad oder einer bestimmten Domänenkonfiguration. Thermodynamische Größen wie die spezifische Wärme sind dagegen zwar eine Volumengröße, ermöglichen aber keine richtungsabhängigen Untersuchungen. Die für jede geordnete Phase zentrale Frage nach dem korrekten Ordnungsparameter ist deshalb im Fall der nematischen Phase von $\mathrm{Sr}_{3} \mathrm{Ru}_{2} \mathrm{O}_{7}$ noch nicht abschließend beantwortet. 
Mit der Dilatometrie, also der empfindlichen Messung kleiner Längenänderungen als Funktion von Temperatur oder Magnetfeld, gibt es allerdings eine Methode, die thermodynamische Informationen sondiert und gleichzeitig richtungsempfindlich ist. Da eine starke Kopplung der kritischen Freiheitsgrade an das Gitter bekannt ist, sollte sich die nematische Phase also gut durch dilatometrische Experimente charakterisieren lassen. $\mathrm{Zu}$ diesem Zweck kommt in der vorliegenden Arbeit eine miniaturisierte kapazitive Ausdehnungsmeßzelle zum Einsatz, mittels derer eine Rotation der untersuchten Probe um verschiedene Achsen auch unter den eingeschränkten Platzverhältnissen in einem ${ }^{3} \mathrm{He}-{ }^{4} \mathrm{He}-$ Mischungskryostaten möglich ist. Durch die Untersuchung von thermischer Ausdehnung und Magnetostriktion in unterschiedlichen kristallographischen Richtungen bei gleichzeitiger Variation des Magnetfeldwinkels soll die Arbeit einen Beitrag zum besseren Verständnis der nematischen Eigenschaften in $\mathrm{Sr}_{3} \mathrm{Ru}_{2} \mathrm{O}_{7}$ leisten.

Die Arbeit ist wie folgt gegliedert: In Kapitel 2 wird zunächst ein Abriß der wichtigsten theoretischen Grundlagen gegeben. Dabei wird auf die Physik von klassischen Phasenübergängen und Quantenphasenübergängen eingegangen und die Thermodynamik der untersuchten Meßgrößen behandelt. Mit dem Prinzip der kapazitiven Dilatometrie befaßt sich Kapitel 3, wobei der verwendete experimentelle Aufbau erläutert und Meßgenauigkeit und Hintergrundeffekte diskutiert werden. Kapitel 4 gibt einen Überblick über die bisherigen Arbeiten zum System $\mathrm{Sr}_{3} \mathrm{Ru}_{2} \mathrm{O}_{7}$. Insbesondere wird auf die experimentelle Evidenz für quantenkritisches Verhalten und die Existenz einer „nematischen Phase" sowie mögliche Modelle für dieselbe eingegangen. Die Frage nach dem korrekten Ordnungsparameter motiviert die in Kapitel 5 vorgestellten Messungen von thermischer Ausdehnung und Magnetostriktion, die sodann im Rahmen eines kürzlich veröffentlichten theoretischen Modells diskutiert werden. Schließlich sollen in Kapitel 6 die Ergebnisse zusammengefaßt und in den wissenschaftlichen Gesamtzusammenhang eingeordnet werden. 



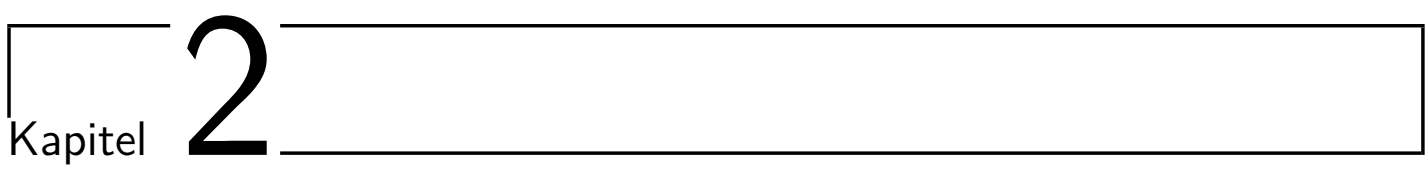

\section{Theoretische Grundlagen}

Da sich diese Arbeit mit einem itineranten System befaßt, soll in diesem Abschnitt zunächst die Theorie der Fermiflüssigkeit behandelt werden, welche die Standardtheorie der Metalle darstellt. Abweichungen von ihren Vorhersagen gehören zu den wichtigsten Effekten, die im Zusammenhang mit Quantenphasenübergängen auftreten, auf die danach zusammen mit ihren klassischen Gegenstücken eingegangen werden soll. Zum Schluß sollen für die untersuchten Meßgrößen die wichtigsten thermodynamischen Beziehungen vorgestellt werden.

\subsection{Fermiflüssigkeits-Theorie}

Die physikalische Beschreibung eines Metalls stellt grundsätzlich betrachtet ein kompliziertes Vielteilchenproblem dar, in welchem die Coulombwechselwirkung zwischen einer Zahl von etwa $10^{23}$ geladenen Teilchen berücksichtigt werden muß. Trotzdem kann die Sommerfeldtheorie des freien Elektronengases, welche die Elektronen unter Vernachlässigung der Wechselwirkung als freie quantenmechanische Teilchen annimmt, die dem Pauliprinzip gehorchen, einfache Metalle erstaunlich gut beschreiben AM76. Die spezifische Wärme und die elektrische Leitfähigkeit werden dabei von Anregungsprozessen in der Nähe der Fermienergie bestimmt.

LANDAU Lan57 erkannte nun, daß dieses Bild auch gültig bleibt, wenn starke elektronische Korrelationen vorliegen. Dabei existiert eine eineindeutige Abbildung zwischen den Eigenzuständen des freien und denen des wechselwirkenden Systems. Die elementaren Anregungen sind im wechselwirkenden Fall nicht mehr einzelne Elektronen, sondern die sog. Quasiteilchen. Obwohl die Anregung ein kollektiver Prozeß ist, wird sie durch die gleichen Quantenzahlen beschrieben wie ein freies Elektron, besitzt also eine Ladung $e$ und einen Spin $\hbar / 2$. Der Effekt der Wechselwirkung äußert sich in einer renomierten Masse $m^{*}$, die i. A. größer als die Masse $m_{e}$ eines freien Elektrons ist. Ein anschauliches Bild eines solchen Quasiteilchens ist ein Elektron, welches aus der Fermikugel angeregt wird und dabei eine „Wolke“ von weiteren Elektronen mit sich 
zieht. In Analogie zu klassischen Systemen wird so aus dem Fermigas eine Fermiflüssigkeit (FF). Zu den wichtigsten Vorhersagen BP91 der FF-Theorie gehören die in der Temperatur lineare spezifische Wärme,

$$
c_{V}=\gamma T=\frac{\pi^{2}}{3} k_{\mathrm{B}}^{2} D\left(E_{\mathrm{F}}\right) T
$$

wobei die Zustandsdichte an der Fermikante $D\left(E_{\mathrm{F}}\right)=m^{*} k_{\mathrm{F}} / \hbar^{2} \pi^{2}$ und damit der Sommerfeldkoeffizient $\gamma$ gegenüber einem freien Elektronengas um einen Faktor $m^{*} / m_{e}$ erhöht ist, die magnetische Suszeptibilität

$$
\chi=\frac{\left(g \mu_{\mathrm{B}}\right)^{2}}{4 \pi^{2}} \frac{m^{*} k_{\mathrm{F}}}{1+F_{0}^{\mathrm{a}}},
$$

die ebenfalls durch die Quasiteilchenmasse $m^{*}$ bestimmt ist, sowie ein quadratisch von der Temperatur abhängiger spezifischer Widerstand

$$
\varrho(T)=A T^{2} .
$$

Das Wechselwirkungspotential $u_{\vec{k} \vec{k}^{\prime}}$ zwischen den Quasiteilchen ist bei einer sphärischen Fermifläche nur vom Winkel zwischen den Wellenvektoren $\vec{k}$ und $\overrightarrow{k^{\prime}}$ abhängig und wird deshalb meist nach Legendrepolynomen entwickelt und in einem relativ kleinen Satz von dimensionslosen Parametern $F_{l}^{\mathrm{s}, \mathrm{a}}$ zusammengefaßt, wobei die Indizes s und a die Wechselwirkung zwischen Quasiteilchen mit gleichem und unterschiedlichem Spin bezeichnen. Viele Eigenschaften der Fermiflüssigkeit lassen sich dann als Funktion dieser Landauparameter ausdrücken: so läßt sich $m^{*}$ beispielsweise als eine Funktion von $F_{1}^{\mathrm{s}}$ darstellen mit $m^{*} / m_{e}=1+1 / 3 F_{1}^{\mathrm{s}}$. Die Theorie der Fermiflüssigkeit ist phänomenologisch in dem Sinne, daß die Werte der $F_{l}^{\mathrm{s}, \mathrm{a}}$ für konkrete Systeme nicht unmittelbar bekannt sind, sondern experimentell bestimmt werden müssen, beispielsweise $F_{1}^{\mathrm{s}}$ durch eine Messung der spezifischen Wärme.

Die effektive Masse $m^{*}$ kann in bestimmten intermetallischen Verbindungen, den sog. Schwere-Fermionen-(SF-)Systemen, enorme Werte von bis zu $1000 m_{e}$ erreichen, was sich in stark erhöhten Werten von $\gamma$ und $\chi$ äußert. Um verschiedene Systeme miteinander zu vergleichen, ist die Betrachtung von dimensionslosen Quotienten zweckmäßig, in denen sich die effektive Masse gerade herauskürzt. Ein Beispiel hierfür ist das Sommerfeld-Wilson-Verhältnis

$$
R_{\mathrm{W}}=\frac{4 \pi^{2} k_{\mathrm{B}}}{3 \hbar^{2}\left(g \mu_{\mathrm{B}}\right)^{2}} \frac{\chi}{\gamma}=\frac{1}{1+F_{0}^{\mathrm{a}}},
$$

das für das nichtwechselwirkende System den Wert 1 hat. $R_{\mathrm{W}}$ ermöglicht den Zugang zum Landauparameter $F_{0}^{\mathrm{a}}$, der die Austauschwechselwirkung beschreibt und damit ein Maß für die Stärke von ferromagnetischen Korrelationen über den Stoner-Mechanismus ist. Auch der Koeffizient $A$ ist i. A. stark erhöht; es ist nämlich bekannt, daß das Kadowaki-Woods-Verhältnis $R_{\mathrm{KW}}=A / \gamma^{2}$ für unterschiedliche Systeme eine Konstante ist. 
Steglichs Entdeckung der Schwere-Fermionen-Supraleitung in $\mathrm{CeCu}_{2} \mathrm{Si}_{2}$ Ste79] zeigte, daß nicht nur Elektronen, sondern auch schwere Quasiteilchen Cooperpaare bilden können und löste ein großes Forschungsinteresse auf dem Gebiet der SF-Systeme aus. Außerdem haben sich SF-Systeme als hervorragend geeignet für die Untersuchung quantenkritischer Phänomene erwiesen [GSS08].

Die Landausche Fermiflüssigkeits-Theorie ist die Standardtheorie der Metalle und eine der wichtigsten Entwicklungen in der theoretischen Festkörperphysik. Auch in Fällen, in denen sie keine Gültigkeit besitzt, ist sie eine wichtige Referenz, an der das Verhalten von unkonventionellen Systemen gemessen werden kann. ${ }^{1}$ Abweichende Effekte werden allgemein unter der Kategorie Nicht-Fermiflüssigkeits-(NFF-) Verhalten zusammengefaßt.

FF-Verhalten findet sich in so unterschiedlichen Systemen wie metallischen Festkörpern, flüssigem ${ }^{3} \mathrm{He}$ oder auch Neutronensternen. ${ }^{2}$ Für eine zugängliche Einführung in die Theorie der Fermiflüssigkeit sei auf BPM10] verwiesen.

\subsection{Klassische Phasenübergänge}

Ein physikalisches System kann je nach äußeren Parametern wie Temperatur oder Druck in unterschiedlichen Phasen vorliegen, welche sich in ihren makroskopischen Eigenschaften grundsätzlich unterscheiden. Eine Klassifizierung der Übergänge zwischen diesen Phasen geht auf Ehrenfest zurück: Danach ist die Ordnung eines Phasenübergangs die Ordnung des niedrigsten Differenzenquotienten des thermodynamischen Potentials, welcher eine Diskontinuität aufweist.

Der Wechsel des Aggregatzustands von flüssig nach fest bei der Siedetemperatur $T_{\mathrm{c}}$ ist beispielsweise mit einer latente Wärme $\Delta Q=T_{\mathrm{c}} \Delta S$ verbunden. Daraus ist direkt ersichtlich, daß die Entropie $S$ bei $T_{\mathrm{c}}$ einen Sprung macht, mit $S=(\partial F / \partial T)_{V}$ ist also eine erste Ableitung der freien Energie $F$ unstetig. Es handelt sich daher um einen Phasenübergang erster Ordnung, wobei die spezifische Wärme $c_{V}=T(\partial S / \partial T)_{V}$ divergiert. Bei einem Übergang zweiter Ordnung sind dagegen die ersten Ableitungen von $F$ stetig und es gibt dementsprechend keine latente Wärme. Erst die zweiten Ableitungen werden diskontinuierlich.

In der Praxis genügen insbesondere Phasenübergänge zweiter Ordnung meist nicht der Ehrenfestklassifikation, denn man beobachtet in den zweiten Ableitungen der freien Energie, wie der spezifischen Wärme $c$ oder der thermischen Ausdehnung $\alpha$, meist eher Singularitäten als echte Sprünge. Als Beispiel sei der Lambda-Übergang des flüssigen ${ }^{4} \mathrm{He}$ zwischen der normal- und suprafluiden Phase genannt: Obwohl die spezifische Wärme divergiert ${ }^{3}$, handelt es sich nicht um einen Phasenübergang erster Ordnung, denn

\footnotetext{
${ }^{1}$ Beispielsweise wird häufig die Größe $\gamma=c_{V} / T$ betrachtet und als Sommerfeldkoeffizient bezeichnet, auch wenn Gleichung 2.1) nicht erfüllt ist. $\gamma$ kann dann bspw. temperaturabhängig werden.

${ }^{2}$ Bei tiefen Temperaturen zeigt ${ }^{3} \mathrm{He}$ eine in $T$ lineare spezifische Wärme mit erhöhtem $\gamma$, was historisch die Motivation zur Entwicklung der FF-Theorie gab.

${ }^{3}$ Die Kurve $c(T)$ beschreibt die namensgebende Form des griechischen Buchstaben $\lambda$.
} 

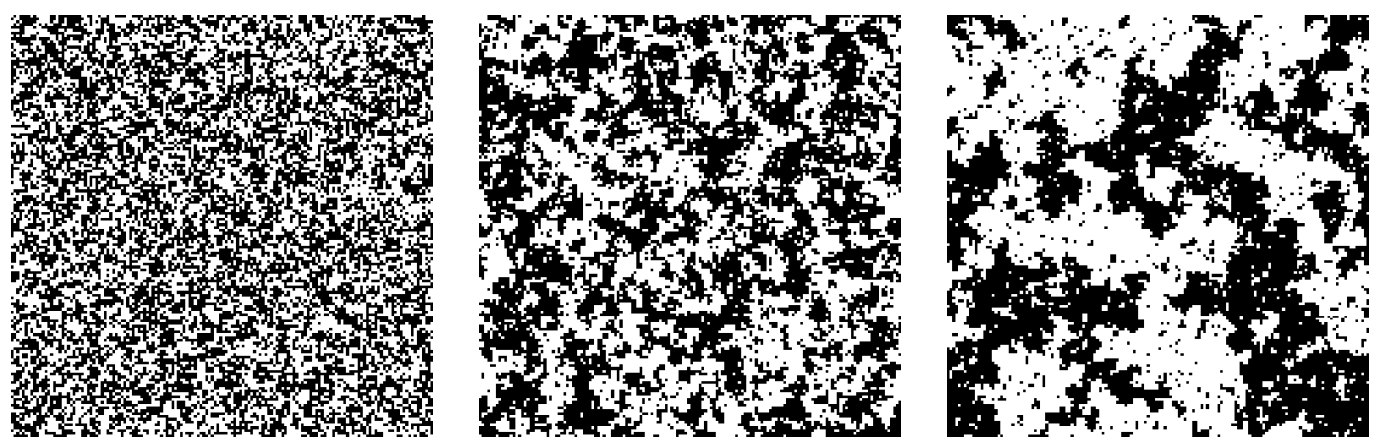

Abbildung 2.1: Monte-Carlo-Simulation des zweidimensionalen Isingmodells. Bei hohen Temperaturen $\left(T=3 T_{\mathrm{c}}\right.$, links) ist das System ungeordnet und die Korrelationslänge $\xi$ ist sehr klein. Bei Annäherung an den Phasenübergang $\left(T=1,2 T_{\mathrm{c}}\right.$, Mitte) wächst $\xi$, um schließlich zu divergieren $\left(T=T_{\mathrm{c}}\right.$, rechts). Quelle: Eigene Simulation.

es wird keine latente Wärme beobachtet. Eines der seltenen Beispiele für einen Phasenübergang zweiter Ordnung im strengen ehrenfestschen Sinn ist dagegen der Übergang eines klassischen Supraleiters im Nullfeld, der einen Sprung in der spezifischen Wärme aufweist.

Da außerdem größere Ordnungen als die zweite in der Realität keine Rolle spielen, unterscheidet man gewöhnlich lediglich zwei Arten von Phasenübergängen: Übergange erster Ordnung bezeichnet man als diskontinuierliche Phasenübergänge, die sich durch Unstetigkeiten in einer ersten partiellen Ableitung der freien Energie auszeichnen. Kontinuierliche Phasenübergänge sind zweiter Ordnung: hier bleiben die ersten partiellen Ableitungen stetig, während Nichtanalytizitäten in Form von Sprüngen, Divergenzen oder Singularitäten in den partiellen Ableitungen zweiter Ordnung auftreten.

Neben dem thermodynamischen Verhalten äußert sich ein Phasenübergang häufig in einer Änderung der Symmetrie des Systems. Eine Flüssigkeit besitzt beispielsweise völlige Translations- und Rotationssymmetrie, da keine Fernordnung zwischen den Teilchen existiert. Beim Erstarren erniedrigt sich diese Symmetrie zu der eines Kristalls, man spricht auch von einer spontanen Symmetriebrechung. Ein anderes Beispiel ist das Verhalten eines Ferromagneten bei Unterschreiten der Curietemperatur: hier kommt es zu einer spontanen Ordnung der magnetischen Momente. Der Magnetisierungsvektor $\vec{M}$ ist eine ausgezeichnete Richtung, womit die Isotropie des Systems gebrochen wird.

Nach LANDAU werden Phasenübergänge häufig durch einen Ordnungsparameter $\Phi$ charakterisiert, der im ungeordneten Zustand gleich 0 und in der geordneten Phase endlich ist. Dies ist beim Übergang flüssig-gasförmig z. B. der Dichteunterschied $\varrho-\varrho_{\text {gas }}$ oder beim Ferromagneten die Magnetisierung $\vec{M}$.

Kontinuierliche Phasenübergänge sind in der Physik von besonderem Interesse, da sie von kritischen Fluktuationen getrieben werden. Während in der ungeordneten Phase der Mittelwert des Ordnungsparameters $\langle\Phi\rangle=0$ ist, sind lokale Fluktuationen um diesen Mittelwert zulässig. Diese Fluktuationen sind durch eine Längenskala $\xi$, die Korrelationslänge und eine Zeitskala $\tau$, die Korrelationszeit, charakterisiert. Mit 
Annäherung an den Phasenübergang divergieren die Fluktuationen als Funktion der reduzierten Temperatur $t=\left(T-T_{\mathrm{c}}\right) / T_{\mathrm{c}}$ in vielen Fällen gemäß einem Potenzgesetz:

$$
\xi \propto t^{-\nu}, \quad \tau \propto \xi^{z}=t^{-\nu z}
$$

Die Parameter $\nu$ und $z$ heißen kritische Exponenten, wobei man $\nu$ als kritischen Exponent der Korrelationslänge und $z$ als dynamisch-kritischen Exponent bezeichnet. Das Divergieren von $\tau$ bedeutet anschaulich, daß die Fluktuationen mit Annäherung an den kritischen Punkt immer langsamer werden, was auch als critical slowing down bezeichnet wird.

Dieser Effekt ist in Abbildung 2.1 am Beispiel des zweidimensionalen Isingmodells illustriert. Wird der Abstand zur kritischen Temperatur hinreichend klein, $t<10^{-2}$, dann wird $\xi$ sehr groß gegen typische Längenskalen des Systems, die z. B. durch den Gitterparameter oder die Reichweite von Wechselwirkungen gegeben sind. In diesem Bereich wird das kritische Verhalten des Systems vollständig von den Fluktuationen bestimmt, während mikroskopische Details unwichtig werden. Dies hat zur Folge, daß auch andere thermodynamische Observablen wie die spezifische Wärme, der Ordnungsparameter, die magnetische Suszeptibilität oder die Kompressibilität Potenzgesetzen mit zugeordneten kritischen Exponenten gehorchen. ${ }^{4}$ Die kritischen Exponenten sind nicht unabhängig, sondern durch sog. Skalengesetze miteinander verknüpft. Die Werte der kritischen Exponenten hängen nach der 1970 von GRIFFITHS [Gri70] formulierten Universalitätshypothese nur noch von

- der Dimensionalität $d$ des Systems, ${ }^{5}$

- der Dimensionalität $n$ des Ordnungsparameters ${ }^{6}$ sowie

- der Reichweite der Wechselwirkungen

ab. Diese beruht auf der bemerkenswerte Beobachtung, daß auf den ersten Blick völlig unterschiedliche physikalische Systeme in dieselbe Universalitätsklasse fallen und damit identisches kritisches Verhalten zeigen. Tabelle 2.1 zeigt einige kritische Exponenten, die sowohl für theoretische Modelle berechnet als auch an realen Systemen experimentell bestimmt wurden. Obwohl die Universalitätsklasse $d=3, n=1$ so unterschiedliche Systeme wie das 3D-Isingmodell, $\mathrm{CO}_{2}$ am kritischen Punkt und den antiferromagnetischen Kristall $\mathrm{FeF}_{2}$ enthält, ergibt sich eine erstaunlich gute Übereinstimmung der kritischen Exponenten.

\footnotetext{
${ }^{4}$ Die Annahme eines Potenzgesetzes ist in vielen Fällen richtig, aber nicht immer allgemein genug. Beispielsweise beobachtet man auch logarithmische Divergenzen der Form $f(t) \propto \log t^{-1}$. Der kritische Exponent wird deshalb allgemein definiert als $\varphi=-\lim _{t \rightarrow 0} \log f(t) / \log (t)$. Ein Exponent $\varphi=0$ beschreibt also sowohl einen Sprung, $f(t)=A_{ \pm}$für $t= \pm|t|$, als auch eine logarithmische Divergenz in der Meßgröße.

${ }^{5}$ auch als „äußere Dimension“ bezeichnet

${ }^{6}$ auch als „,innere Dimension“ bezeichnet
} 


\begin{tabular}{l|l|l|l|l}
$\begin{array}{l}\text { Universalitäts- } \\
\text { klasse }\end{array}$ & System & $\alpha$ & $\beta$ & $\gamma$ \\
\hline$d=3, n=1$ & $3 \mathrm{~d}-$ Ising-Modell & $0,110(5)$ & $0,3250(15)$ & \\
& $\mathrm{CO}_{2}$ am kritischen Punkt & $0,12(1)$ & 0,321 & \\
& $\mathrm{FeF}_{2}$ (Antiferromagnet) & $0,11(2)$ & $0,325(2)$ & \\
\hline$d=3, n=3$ & $3 \mathrm{~d}-$ Heisenberg-Modell & & 0,365 & $1,3866(12)$ \\
& EuO (Ferromagnet) & & $0,367(8)$ & $1,387(36)$ \\
\hline$d=2, n=1$ & $2 \mathrm{~d}-$ Ising-Modell & $0(\log )$ & $1 / 8=0,125$ & $7 / 4=1,75$ \\
& $\mathrm{~K}_{2} \mathrm{CoF}_{4}$ (Antiferromagnet) & $-0,003(39)(\log )$ & $0,123(8)$ & $1,71(4)$
\end{tabular}

Tabelle 2.1: Innerhalb einer Universalitätsklasse stimmen die kritischen Exponenten $\alpha$ der spezifischen Wärme, $\beta$ des Ordnungsparameters und $\gamma$ der Suszeptibilität für völlig unterschiedliche physikalische Systeme überein. Werte für $\mathrm{CO}_{2}$ aus LEB70 HM76, für $\mathrm{FeF}_{2}$ aus MDN94 MMB95, für EuO aus AN71, für $\mathrm{K}_{2} \mathrm{CoF}_{4}$ aus IH74 Ike75, Rest aus Gol92, GK80.

Theorien Eine phänomenologische Beschreibung von kritischen Phänomenen leistet die Theorie von LANDAU, in der der kritische Anteil der freien Energie in der Nähe von $T_{\mathrm{c}}$ nach dem Ordnungsparameter entwickelt wird. Wie die Weisssche Theorie des Ferromagnetismus (hier ist der Ordnungsparameter die Magnetisierung) zählt sie damit zu den Molekularfeld- oder Mean-Field-Theorien. Sie ist nur selbstkonsistent, solange die Fluktuationen klein gegen den Mittelwert des Ordnungsparameters bleiben, $\left\langle(\Phi-\langle\Phi\rangle)^{2}\right\rangle \ll\langle\Phi\rangle^{2}$. Diese Bedingung, die als Ginzburg-Kriterium bezeichnet wird, ist aber meist verletzt, da sich der kritische Bereich gerade durch starke Fluktuationen auszeichnet. Die Vorhersagen der Landau-Theorie stimmen daher i. A. nicht mit Experimenten überein: Beispielsweise wird für den kritischen Exponenten $\alpha$ der Wert 0 vorhergesagt, also ein scharfer Sprung in der spezifischen Wärme bei $T_{\mathrm{c}}$. In realen Systemen ist dieser Sprung aber meist durch Fluktuationseffekte überlagert. Allerdings ist das Ginzburg-Kriterium für Dimensionen $d>4$ stets erfüllt, so daß hier die Mean-Field-Theorie korrekt wird. Dieser Wert wird als obere kritische Dimension bezeichnet. $^{7}$

WIDOM formulierte 1965 die Skalenhypothese, welche besagt, daß der singuläre Anteil der freien Energiedichte (eines magnetischen Systems) der Form

$$
L^{d} f_{\mathrm{krit}}(t, h)=f_{\mathrm{krit}}\left(L^{x} t, L^{y} h\right)
$$

gehorcht $\mathrm{McC04}$. Eine Reskalierung aller Längen um den Faktor $L$ läßt das thermodynamische Potential unverändert, solange reduzierte Temperatur $t=\left(T-T_{\mathrm{c}}\right) / T_{\mathrm{c}}$ und Feld $h$ (bzw. die Kopplungskonstanten) ebenfalls geeignet reskaliert werden und $L \ll \xi$ gilt, also der Skalierungsfaktor klein gegen die Korrelationslänge als einzige relevante Längenskala bleibt. Aus (2.6) lassen sich die Skalengesetze ableiten, wobei alle kritischen Exponenten durch die Exponenten $x$ und $y$ ausgedrückt werden können. Eine explizite Methode für eine solche Reskalierung hatte erstmals KaDANOFF 1966

\footnotetext{
${ }^{7}$ Analog existiert eine untere kritische Dimension, unterhalb derer Fluktuationen so stark sind, daß keine Ordnung bei $T>0$ stattfindet.
} 


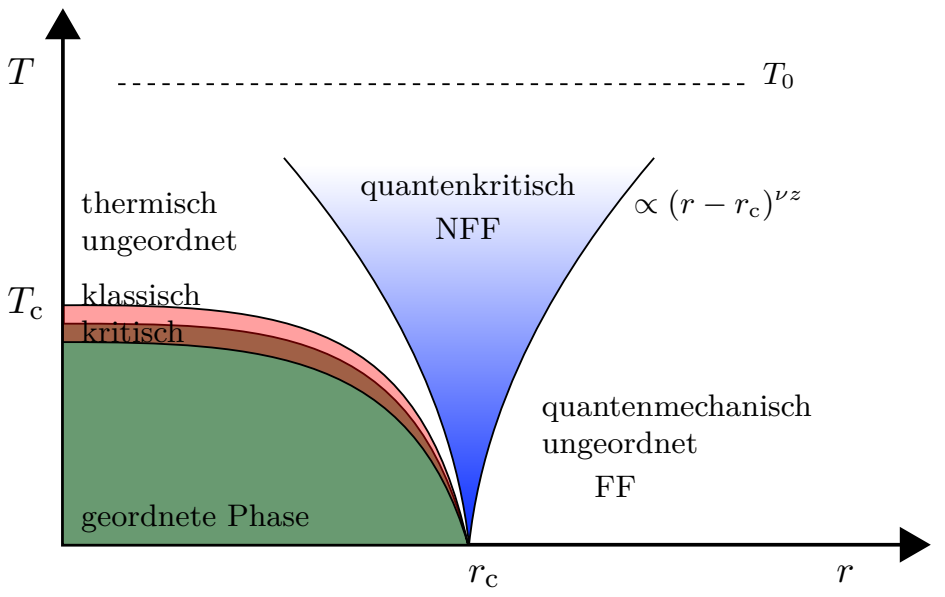

mit seiner Blockspinkonstruktion angegeben. Der zentrale Gedanke ist hier gerade die Selbstähnlichkeit des Systems auf allen Längenskalen.

Die Gültigkeit der Universalitäts- und der Skalenhypothese läßt sich im Rahmen von WiLsons Renormierungsgruppentheorie (RGT, Nobelpreis 1982) exakt begründen Gol92; McC04. Sie ermöglichte erstmals die Berechnung der kritischen Exponenten von nicht exakt lösbaren Modellen. In der modernen theoretischen Physik stellt die RGT das wichtigste Werkzeug zur Behandlung kritischer Phänomene dar.

\subsection{Quantenphasenübergänge}

Es stellt sich nun die Frage, welchen Einfluß quantenmechanische Effekte auf einen Phasenübergang haben. Einerseits ist die geordnete Phase oft nur quantenmechanisch zu verstehen (z. B. im Fall von Supraleitung oder Magnetismus), andererseits wird das kritische Verhalten am Phasenübergang nicht nur durch thermische, sondern auch durch quantenmechanische Fluktuationen mit der Frequenz $\omega_{\mathrm{c}}$ beeinflußt. Um zu entscheiden, welche Art von Fluktuationen dominiert, muß man die thermische Energie $k_{\mathrm{B}} T$ mit der Quantenenergie $\hbar \omega_{\mathrm{c}} \propto 1 / \tau \propto t^{\nu z}$ vergleichen. Da die Korrelationszeit $\tau$ für $T \rightarrow T_{\mathrm{c}}$ divergiert, geht $\hbar \omega_{\mathrm{c}} \rightarrow 0$, so daß für $T_{\mathrm{c}}>0$ immer die thermischen Fluktuationen dominieren. Alle Phasenübergänge, die bei endlicher Temperatur stattfinden, können deshalb als klassisch bezeichnet werden |Voj03.

Nur, wenn die Übergangstemperatur tatsächlich zu $T_{\mathrm{c}}=0$ reduziert wird, können Quantenfluktuationen dominieren. In diesem Fall spricht man von einem Quantenphasenübergang (QPÜ). Zwar verbietet der dritte Hauptsatz der Thermodynamik das experimentelle Erreichen des absoluten Temperaturnullpunkts, trotzdem ist das Konzept eines QPÜs nicht nur von theoretischem Interesse, wie das generische Phasendiagramm in Abbildung 2.2 zeigt.

Unterhalb einer Ordnungstemperatur $T_{\mathrm{c}}$ existiert eine geordnete Phase, die häufig magnetischer Natur ist. $T_{\mathrm{c}}$ ist hierbei eine Funktion eines externen Kontrollparame- 
ters $r$. Liegen lokalisierte magnetische Momente vor, kann beispielsweise durch Anlegen eines hydrostatischen Drucks der orbitale Überlapp zwischen den Wellenfunktionen erhöht werden, was die Delokalisierung der Elektronen begünstigt und den Magnetismus schließlich zerstört. Ein antiferromagnetischer Zustand kann dagegen durch ein externes Magnetfeld unterdrückt werden. Ein weiteres Beispiel für einen Kontrollparameter ist die Dotierung durch isoelektronische Fremdatome, die nicht die Ladungsträgerkonzentration, aber durch ihren unterschiedlichen Ionenradius die Gitterkonstante des Systems ändern. Dies wird auch als Anlegen eines chemischen Drucks bezeichnet. Im Gegensatz zu hydrostatischem Druck läßt sich durch Dotierung die Gitterkonstante auch aufweiten, also ein effektiver negativer Druck ausüben.

Wird der Kontrollparameter $r$ nun auf einen kritischen Wert $r_{\mathrm{c}}$ eingestellt, so wird $T_{\mathrm{c}}=0$ und man erhält einen quantenkritischen Punkt (QKP). Für hohe $T$ und kleine $r$ wird das System von klassischen thermischen Fluktuationen dominiert: in einem Bereich $t \leq 10^{-2}$ zeigt sich klassisch kritisches Verhalten und für höhere $T$ wird die Ordnung durch thermische Fluktuationen zerstört, während sich für größere $r$ ein quantenmechanisch ungeordneter Bereich ergibt. Besonders interessant ist nun der quantenkritische Bereich, der durch $k_{\mathrm{B}} T \geq \hbar \omega_{\mathrm{c}} \propto\left(r-r_{\mathrm{c}}\right)^{\nu z}$ begrenzt ist. Das System ist hier charakterisiert durch die thermische Anregung des quantenkritischen Grundzustands. Die Anregungen lassen sich nicht mehr als konventionelle Quasiteilchen beschreiben [Voj03, was anomales Verhalten bei endlichen Temperaturen hervorruft wie unkonventionelle kritische Exponenten oder Nicht-Fermiflüssigkeits-(NFF-)Verhalten.

Häufig beobachtet man bei der Annäherung an einen QKP die Bildung von neuartigen geordneten Phasen. Das berühmteste Beispiel dafür ist die Supraleitung in den schwere-Fermionen-Systemen $\mathrm{CePd}_{2} \mathrm{Si}_{2}$ und $\mathrm{CeIn}_{3}$ Mat98. In diesen Systemen wird ein antiferromagnetischer Zustand durch externen Druck zu $T=0$ unterdrückt und in einer Kuppel über dem QKP Supraleitung gefunden. Am QKP ist das System gerade an der Schwelle einer spontanen magnetischen Ordnung, so daß Spinwellen sehr leicht angeregt werden können. Man geht deshalb von einem magnetischen Mechanismus der Cooperpaarung zwischen den schweren Quasiteilchen aus Mat98. Weitere Kandidaten sind die Hochtemperatursupraleiter (HTSL) auf CuO-Basis, deren Phasendiagramm zwar komplizierter ist als das generische in Abbildung 2.2, aber trotzdem Gemeinsamkeiten aufweist. Im undotierten Zustand sind die HTSL antiferromagnetische Mott-Isolatoren, in denen durch Lochdotierung die charakteristische Temperatur $T^{*}$ der sog. Pseudogap-Phase zu $T^{*}=0$ unterdrückt wird. Dieser Punkt liegt in der Nähe der optimalen Dotierung, d.h. der höchsten Übergangstemperatur, weswegen man unter der supraleitenden Kuppel einen verborgenen QKP vermutet [Bro08]. Die Erforschung von quantenkritischen Phänomenen könnte damit auch zur Klärung des bis heute unverstandenen Mechanismus der Supraleitung in den HTSL-Materialien beitragen.

Theorien Ein thermodynamisches System, welches durch eine Hamiltonfunktion

$$
H=H_{\text {kin }}+H_{\text {pot }}
$$




\begin{tabular}{c|c|c} 
& quantenkritischer Bereich & FF-Bereich \\
\hline$\alpha$ & $\log \frac{1}{T}$ & $\operatorname{Tr}^{-3 / 2}$ \\
$c$ & $T^{2 / 3}$ & $\operatorname{Tr}^{-1 / 2}$
\end{tabular}

\begin{tabular}{c|c}
\multicolumn{2}{c}{$\mathrm{mm}$ QKEP } \\
\hline$\chi$ & $r^{-2 / 3}$ \\
$c$ & $T r^{-1 / 3}$ \\
$\varrho=\varrho_{0}+A T^{2}$ & $A \propto r^{-2 / 3}$
\end{tabular}

Tabelle 2.2: Links: Vorhersagen der Hertz-Millis-Theorie für das kritische Verhalten der thermischen Ausdehnung und der spezifischen Wärme eines zweidimensionalen Ferromagneten $(d=2$ und $z=3)$, aus Zhu03]. Rechts: Vorhersagen für den metamagnetischen quantenkritischen Endpunkt mit Kontrollparameter $r=\left(H-H_{\mathrm{c}}\right) / H_{\mathrm{c}}$, aus Mil02.

beschrieben wird, gilt als gelöst, wenn die Zustandssumme $Z=\operatorname{Tr} \exp (-\beta H)$ bekannt ist. Da im klassischen Fall der potentielle und kinetische Anteil von $H$ vertauschen, faktorisiert die Zustandssumme, $Z=Z_{\mathrm{kin}} \cdot Z_{\mathrm{pot}}$, so daß Statik und Dynamik entkoppeln. Im quantenmechanischen Fall vertauschen die Operatoren für die kinetische und potentielle Energie jedoch i. A. nicht, so daß die zeitliche Dynamik explizit berücksichtigt werden muß.

Der Zeitentwicklungsoperator $\exp (-i H t / h)$ läßt sich durch Einführung einer imaginären Zeit $\theta=-i \hbar \beta$ in die Form des statistischen Operators $\exp (-\beta H)$ (mit $\beta=$ $\left.1 / k_{\mathrm{B}} T\right)$ bringen. Dadurch kann ein quantenmechanisches Modell in ein äquivalentes klassisches Modell überführt werden, in welchem die imaginäre Zeitachse eine zusätzliche Dimension der Länge $\theta$ darstellt [Son97]. Die Temperatur des zugeordneten klassischen Systems ergibt sich dabei aus den Kopplungskonstanten des quantenmechanischen Systems, während die physikalische Temperatur nur über die Größe des Systems in der Zeitrichtung eingeht. Da die Zeit nach Gleichung (2.5) wie die $z$-te Potenz einer Länge skaliert, ergibt sich eine effektive Dimension

$$
d_{\mathrm{eff}}=d+z .
$$

Dies ist für die theoretische Beschreibung ein vorteilhaftes Ergebnis, da $d_{\text {eff }}$ möglicherweise über der oberen kritischen Dimension von Mean-Field-Theorien liegt, die damit wieder gültig werden.

Die erste theoretische Untersuchung einer magnetischen Instabilität in itineranten Elektronensystemen bei $T=0$ stammt von HeRTz [Her76] und wurde später von MiLlis Mil93 für endliche Temperaturen erweitert. Dabei wird das kritische Verhalten mit einer störungstheoretischen Renormierungsgruppenmethode behandelt. Ist die geordnete Phase ferromagnetischer Natur, so ergibt sich ein dynamisch-kritischer Exponent von $z=3$ und für den antiferromagnetischen Fall $z=2$. Eine der wichtigsten Vorhersagen [Löh07] der Hertz-Millis-(HM-)Theorie ist NFF-Verhalten im quantenkritischen Bereich mit einem anomalen Widerstandsexponenten kleiner als 2. Dies ist in einer großen Zahl von Systemen realisiert.

In der HM-Theorie sind (wie im klassischen Fall) die einzigen kritischen Fluktuationen die des magnetischen Ordnungsparameters. Es sind allerdings auch Systeme bekannt, in denen weitere Freiheitsgrade existieren: In $\mathrm{YbRh}_{2} \mathrm{Si}_{2}$ ist beispielsweise die Annäherung an den QKP mit einem Zusammenbruch des Kondoeffekts und einer 
sprunghaften Änderung des Fermiflächenvolumens verbunden. Damit gibt es zusätzliche kritische Moden und dementsprechend auch mehr als eine relevante Energieskala [Geg07]. Ein solcher QKP wird durch die HM-Theorie nicht mehr korrekt beschrieben, vielmehr spricht man von einem „lokalen quantenkritischen Szenario“ GSS08.

Der Test für die Theorie ist gewöhnlich das Verhalten verschiedener thermodynamischer Meßgrößen im quantenkritischen und im FF-Bereich. Ein Beispiel für die Vorhersagen für $d=2$ und $z=3$ zeigt Tabelle 2.2 (links). Ein Überblick über die Ergebnisse für andere Werte von $d$ und $z$ findet sich in Gar03 und Küc05.

Während die Hertz-Millis-Theorie für einen itineranten Ferromagneten im Nullfeld nicht gültig ist Gar03, zeigten MILLIS et al. [Mil02], daß sie für einen ferromagnetischen Übergang erster Ordnung im Magnetfeld ihre Gültigkeit mit gewissen Korrekturen behält. Die Hertz-Millis-Theorie mit $z=3$ ist damit die korrekte Beschreibung für einen metamagnetischen quantenkritischen Endpunkt, wie er im Fall des in dieser Arbeit untersuchten Systems $\mathrm{Sr}_{3} \mathrm{Ru}_{2} \mathrm{O}_{7}$ realisiert ist. Tab. 2.2 (rechts) zeigt für diesen Fall die aus Mil02] entnommenen Vorhersagen. Die genaue Theorie für $\alpha$ ist in Geg06b angegeben und wird in Abschnitt 4.3 besprochen.

\subsection{Thermodynamische Meßgrößen}

In diesem Abschnitt sollen einige thermodynamische Größen behandelt werden, die zur Charakterisierung von Quantenphasenübergängen bevorzugt untersucht werden. Es werden wichtige Relationen angegeben sowie das erwartete Verhalten in klassischen und quantenkritischen Fällen diskutiert.

Thermische Ausdehnung Die thermischen Ausdehnungskoeffizienten $\alpha$ und $\beta$ sind die Ableitungen der relativen Längen- bzw. Volumenänderungen nach der Temperatur,

$$
\alpha=\frac{1}{L}\left(\frac{\partial L}{\partial T}\right)_{p} \quad \text { und } \quad \beta=\frac{1}{V}\left(\frac{\partial V}{\partial T}\right)_{p}
$$

für isotrope Systeme gilt $\alpha=3 \beta$. Die thermische Ausdehnung eines Festkörpers wird durch zwei Beiträge bestimmt: Zum einen führt die Anharmonizität des Bindungspotentials bei Anregung von Gitterschwingungen zu einem erhöhten mittleren Ionenabstand. Unter der Annahme, daß die Frequenz aller Phononenmoden in gleicher Weise vom Volumen abhängt, ist die thermische Ausdehnung proportional zum Phononenanteil der spezifischen Wärme, $\beta=(1 / B) \gamma c_{V}^{\text {phon }}$, wobei der Grüneisenparameter ${ }^{8} \gamma$ eine schwach temperaturabhängige Konstante der Größenordnung 1 ist [AM76]. Ein zweiter Beitrag stammt von der kinetischen Energie $U$ des Elektronensystems, das im

\footnotetext{
${ }^{8}$ Der Grüneisenparameter $\gamma$ beschreibt in der Darstellung von AM76 lediglich die Auswirkungen phononischer Effekte. Er ist deshalb zu unterscheiden vom unten diskutierten Grüneisenverhältnis $\Gamma$, welches über thermodynamische Relationen des ganzen Systems definiert ist und damit auch elektronische Effekte einschließt.
} 
Fall von freien Elektronen einen Druck $p=2 / 3(U / V)$ auf das Gitter ausübt, der zur Aufweitung führt. Mit dem Elastizitätsmodul $\mathcal{E}=\partial p / \partial V)_{T}$ ergibt sich

$$
\beta=\frac{1}{\mathcal{E}}\left(\gamma c_{V}^{\mathrm{phon}}+\frac{2}{3} c_{V}^{\mathrm{el}}\right),
$$

man erwartet also für die thermische Ausdehnung als Funktion der Temperatur ein ähnliches Verhalten wie für die spezifische Wärme. Insbesondere sollte sich eine Konstante für hohe Temperaturen, $\beta \propto T^{3}$ für $T$ im Bereich der Debyetemperatur $\Theta_{\mathrm{D}}$ und $\beta \propto T$ für $T \ll \Theta_{\mathrm{D}}$ ergeben. Bei Tieftemperaturmessungen ist vor allem der letzte Bereich von Interesse, in welchem die thermische Ausdehnung von elektronischen Effekten dominiert ist. Schwere-Fermionen-Systeme zeigen dort extrem große thermische Ausdehnung, denn einerseits erhöht die Renormierung der effektiven Masse neben der spezifischen Wärme auch die thermische Ausdehnung um einen Faktor $m^{*} / m$ im Vergleich zu einfachen Metallen. Andererseits kann auch das unten behandelte Grüneisenverhältnis große Werte annehmen und so $\beta$ gegenüber $c$ zusätzlich erhöhen.

Magnetostriktion Die Magnetostriktion $\lambda$ beschreibt die Längen- oder Volumenänderung als Funktion eines äußeren Magnetfelds $B$,

$$
\lambda=\frac{1}{V}\left(\frac{\partial V}{\partial B}\right)_{T, p} \stackrel{\text { M.R. }}{=}-\frac{1}{V}\left(\frac{\partial M}{\partial p}\right)_{T, B} .
$$

Die Kraft oder das Drehmoment, welches die Magnetisierung einer Probe im äußeren Magnetfeld erfährt, bewirkt eine Spannung im Material, die wiederum zu einer Dehnung führt. Für ein isotropes, para- oder diamagnetisches Metall ist eine quadratische Abhängigkeit vom Magnetfeld zu erwarten, da $\Delta L \propto H M=\chi H^{2}$. Dehnt sich die Probe parallel zu Magnetfeld und Magnetisierung aus, so erwartet man nach dem Poissoneffekt eine Kontraktion in der dazu senkrechten Richtung. In realen Materialien hängt das Vorzeichen von $\lambda$ von der kristallographischen Richtung ab. Da Spannung und Dehnung durch einen Tensor vierter Ordnung verknüpft sind, kann das magnetostriktive Verhalten äußerst kompliziert sein [O'H00].

Grüneisenverhältnis Die spezifische Wärme

$$
c_{p}=T\left(\frac{\partial S}{\partial T}\right)_{p}
$$

und die thermische Ausdehnung

$$
\beta=\frac{1}{V}\left(\frac{\partial V}{\partial T}\right)_{p} \stackrel{\text { M.R. }}{=}-\frac{1}{V}\left(\frac{\partial S}{\partial p}\right)_{T}
$$

beschreiben die Änderung der Entropie $S$ als Funktion der äußeren Parameter $T$ und $p$. Eine nützliche Größe ist nun der Grüneisenparameter $\Gamma$ mit 


$$
\Gamma=\frac{\beta}{c_{p}}=-\frac{1}{V T} \frac{(\partial S / \partial p)_{T}}{(\partial S / \partial T)_{p}}
$$

Viele Systeme besitzen nämlich bei tiefen Temperaturen eine einzelne Energieskala $E_{0}$, die sie eindeutig charakterisiert. Dies ist für das Elektronensystem beispielsweise die Fermienergie $E_{\mathrm{F}}$, für das Kristallgitter die Debyetemperatur $\Theta_{\mathrm{D}}$ oder für einen Magneten die Kopplungskonstante $J$. Dabei ist $E_{0}=E_{0}(p)$ eine Funktion des Drucks. ${ }^{9}$ Die Entropie läßt sich dann als eine Funktion des Verhältnisses von Temperatur und charakteristischer Energieskala schreiben:

$$
S=f\left(\frac{k_{\mathrm{B}} T}{E_{0}}\right)
$$

So geht beispielsweise im Fall von Phononen $f$ für kleine Argumente gegen 0 (Ausfrieren der Phononen für $T \ll \Theta_{\mathrm{D}}$ und für große Argumente gegen const $T$ (Gesetz von Dulong und Petit für $\left.T \gg \Theta_{\mathrm{D}}\right)$. Mit (2.12) wird (2.11) zu

$$
\Gamma=\frac{k_{\mathrm{B}}}{V E_{0}} \frac{\partial E_{0}}{\partial p}
$$

Der Grüneisenparameter beschreibt also die Abhängigkeit der charakteristischen Energie vom Kontrollparameter. Für einfache Metalle ist $E_{0}$ eine Konstante, das Verhältnis aus thermischer Ausdehnung und spezifischer Wärme also konstant und temperaturunabhängig, vgl. auch 2.8. Dieses Ergebnis ist seit 1908 als Grüneisengesetz bekannt Grü08.

Betrachtet man nun einen QKP und nähert sich ihm als Funktion des Kontrollparameters, so erwartet man sowohl für $\beta$ und $c_{p}$ einen Anstieg, da die Fluktuationen die Entropie erhöhen, aber nicht notwendigerweise ein universelles Verhalten. Als Funktion der Temperatur müssen außerdem beide Größen gegen null gehen, um dem dritten Hauptsatz der Thermodynamik zu genügen. Außergewöhnlich ist nun aber das Verhalten von $\Gamma$ : Die charakteristische Energie $E_{0}$ ist hier nämlich gerade die Energie der Quantenfluktuationen, die aber mit Annäherung an den QKP verschwindet: $E_{0}=\hbar \omega_{\mathrm{c}} \propto \tau^{-1} \rightarrow 0$, so daß der Grüneisenparameter 2.13 also divergiert. Garst Gar03 und ZHu Zhu03 konnten zeigen, daß eine solche Divergenz eine allgemeine Eigenschaft eines quantenkritischen Punkts ist, solange nur die freie Energie einem Skalengesetz gehorcht. Im quantenkritischen Bereich, also bei Annäherung als Funktion der Temperatur (vgl. Abbildung 2.2) gilt demnach

$$
\Gamma \propto T^{-\nu z}
$$

\footnotetext{
${ }^{9}$ Äußerer Druck auf einen Festkörper ändert die Gitterkonstante. Dies wirkt sich wiederum auf die elastische Kopplung zwischen den Atomen und damit auf $\Theta_{D}$ aus oder auf die Kopplungsstärke $J$ zwischen magnetischen Ionen.
} 
während im Fermiflüssigkeitsbereich (Annäherung als Funktion des Kontrollparameters) sogar der Vorfaktor exakt als eine Kombination von kritischen Exponenten bestimmt werden kann:

$$
\Gamma=\frac{\nu\left(d-y_{0} z\right)}{y_{0}} \frac{1}{V_{\mathrm{m}}\left(p-p_{\mathrm{c}}\right)} \propto r^{-1}
$$

Dieses Verhalten wurde erstmals von KüCHLER et al. Küc03 im Schwere-FermionenMetall $\mathrm{CeNi}_{2} \mathrm{Ge}_{2}$ gefunden. Dabei konnte im quantenkritischen Bereich eine Divergenz von $\Gamma$ mit einem Exponenten $\nu z=1$ über mindestens zwei Dekaden in der Temperatur gemessen und so gezeigt werden, daß sich $\mathrm{CeNi}_{2} \mathrm{Ge}_{2}$ im undotierten Zustand auf natürliche Weise an einem antiferromagnetischen QKP befindet. Der gemessene Exponent ist hierbei konsistent mit den Vorhersagen der HM-Theorie $(\nu=1 / 2$ und $z=2)$.

Ist das magnetische Feld $H$ der Kontrollparameter, so läßt sich analog der magnetische Grüneisenparameter

$$
\Gamma_{H}=-\frac{(\partial M / \partial T)_{H}}{c_{H}}=-\frac{1}{\mu_{0} T} \frac{(\partial S / \partial H)_{T}}{(\partial S / \partial T)_{H}}=\frac{1}{\mu_{0} T}\left(\frac{\partial T}{\partial H}\right)_{S}
$$

definieren, welcher ebenso wie $\Gamma$ bei Annäherung an einen QKP divergiert. Dies konnte beispielsweise im System $\mathrm{YbRh}_{2} \mathrm{Si}_{2}$ beobachtet werden [Tok09]. Auch hier wird das Verhältnis der Entropieänderungen in den Richtungen von Kontrollparameter und Temperatur gemessen. Um $\Gamma_{H}$ experimentell zu bestimmen, können nun Magnetisierung und spezifische Wärme separat gemessen und ihr Quotient ermittelt werden. Die rechte Seite von Gleichung (2.16) zeigt aber, daß $\Gamma_{H}$ auch auf elegante Weise direkt aus dem magnetokalorischen Effekt, also der relativen Temperaturänderung bei einer adiabatischen Änderung des Magnetfeldes, erhalten werden kann. TokiwA und GEGENWART [TG11] haben kürzlich eine Wechselfeldmethode zur Messung des magnetokalorischen Effekts entwickelt, die schnellere und präzisere Ergebnisse liefert als die separate Bestimmung von $M$ und $c_{V}$. 



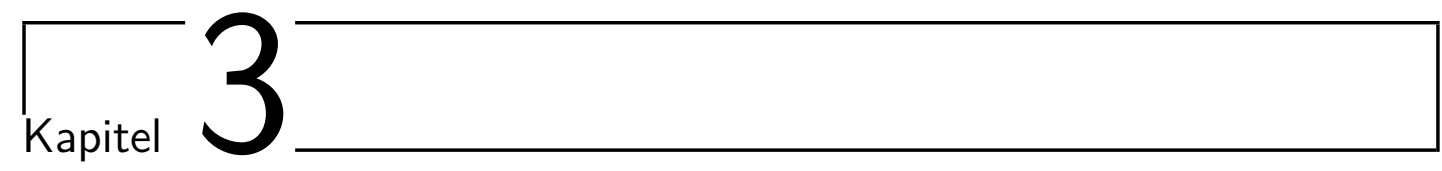

\section{Experimentelle Methoden und Datenanalyse}

\subsection{Erzeugung tiefer Temperaturen}

Für die in dieser Arbeit beschriebenen Tieftemperaturexperimente wurde ein ${ }^{3} \mathrm{He}-{ }^{4} \mathrm{He}-$ Entmischungskryostat vom Typ Kelvinox 100 der Fa. Oxford Instruments eingesetzt. Dabei stehen Temperaturen bis $15 \mathrm{mK}$ zur Verfügung. Die Kühlwirkung in einem solchen System basiert auf der Mischungswärme der zwei Heliumisotope ${ }^{3} \mathrm{He}$ und ${ }^{4} \mathrm{He}$. Für die Erzeugung tiefer Temperaturen über Zeiträume von Stunden bis Monaten ist die ${ }^{3} \mathrm{He}-{ }^{4} \mathrm{He}-$ Mischungskühlung das etablierte Standardverfahren. Eine detaillierte Beschreibung der Funktionsweise findet sich z. B. in den Lehrbüchern von PoBELL Pob07] oder Enss und Hunklinger EH00.

Der Mischungseinsatz ist in einem ${ }^{4} \mathrm{He}$-Badkryostaten mit einem Supraleitungsmagneten, der ein maximales Magnetfeld von $18 \mathrm{~T}$ bereitstellen kann, installiert. Um die Probe oder ganze Experiment auszutauschen, kann der Einsatz separat entnommen werden, ohne den Kryostaten und den Magneten aufwärmen zu müssen.

\subsection{Dilatometrie}

Thermische Ausdehnung und Magnetostriktion werden in dieser Arbeit mit der Methode der kapazitiven Dilatometrie bestimmt. Eine Längenänderung der Probe verändert dabei den Plattenabstand eines Kondensators, dessen Kapazität mit hoher Präzision gemessen wird. Der Aufbau des Dilatometers geht auf Potт und Schefzyk |PS83] zurück, die auch Meßprinzip und -fehler detailliert diskutieren. Die thermische Entkopplung der Probe folgt dem Vorbild von LANG Lan91. 


\subsubsection{Prinzip und Aufbau}

Das hier verwendete Dilatometer wurde von R. KücHLER am Max-Planck-Institut für Chemische Physik fester Stoffe in Dresden entwickelt und in Göttingen teilweise modifiziert. Abbildung 3.1 zeigt einen Schnitt durch die Meßzelle, anhand dessen Aufbau und Funktionsweise erläutert werden sollen.

Der bewegliche Rahmen (1) ist mit zwei Blattfedern (2) am festen Rahmen (3) aufgehängt. Durch die Parallelgeometrie kann sich der Rahmen in vertikaler Richtung bewegen, jedoch nicht verkippen. Eine Ausdehnung der Probe (4) drückt den beweglichen Rahmen gegen die Federkraft nach unten, was den Abstand zwischen den Kondensatorplatten (5) verringert. Die Probe selbst ist über den aus Graphit gefertigten Probenhalter (6) sowie ein weiteres Graphitstück (7) thermisch von der Meßzelle isoliert, dafür jedoch über den Silberstempel (8) und einen Silberzopf (8 Drähte mit einem Durchmesser von je 0,25 mm) direkt an die Mischungskammer bzw. eine Temperaturregelungsplattform angekoppelt. Die abnehmbare Spannvorrichtung (9) mit Spannschraube (10) drückt den Spannstempel (11) nach unten, welcher nach Erreichen der gewünschten Arbeitskapazität mit der Klemmschraube (12) arretiert wird.

Der Zellenkörper ist aus CuBe gefertigt. Dieses Material zeichnet sich einerseits durch eine große Härte aus. Andererseits hat $\mathrm{CuBe}$ einen hohen Restwiderstand, was Aufheizungen durch Wirbelströme bei Änderung des Magnetfelds reduziert. An einem geeigneten Halter läßt sich die Meßzelle mit der seitlichen Befestigungsschraube in unterschiedlichen Winkelstellungen im Vakuumbecher des Kryostaten montieren. Abbildung 3.2 zeigt beispielsweise die Einbaulage zur Messung der Längenänderung senkrecht zum angelegten Magnetfeld, $\Delta L \perp B$.

Bei ersten Probemessungen stellte sich die Thermalisierung einer direkt ins Dilatometer eingebauten Probe als problematisch heraus. Es hat sich deshalb als zweckmäßig erwiesen, die Probe wie oben beschrieben thermisch von der Meßzelle zu isolieren und stattdessen direkt an die Mischungskammer bzw. eine Temperaturregelungsplattform anzukoppeln. Für den Probenhalter bietet sich Graphit als bei tiefen Temperaturen bester bekannter thermischer Isolator an Pob07. Hier wird ein von der Fa. Kestlen IngenieurTechniK bezogener Präzisionsgraphit der Sorte SB811C mit einer mittleren Korngröße von $2 \mu \mathrm{m}$ verwendet, der sich durch gute feinmechanische Bearbeitbarkeit auszeichnet. Der Probenhalter besitzt an seinem unteren Ende einen Vierkant, der in eine passend erodierte Aussparung im Dilatometer greift. Dadurch ist auch eine korrekte Ausrichtung der Probe bezüglich einer Rotation um die Hochachse gewährleistet.

Die Kapazität wird mit einer Meßbrücke vom Typ AH-2500A der Fa. AndeEnHagerling bestimmt. Das Gerät gleicht die zu messende Kapazität mit einem internen temperaturstabilisierten Referenzkondensator ab, wodurch Messungen mit einer Präzision ${ }^{1}$ von $1 \cdot 10^{-7} \mathrm{pF}$ ermöglicht werden. Um das Rauschen gering zu halten, mittelt das Gerät bereits intern über mehrere Meßwerte, nur das Ergebnis dieser Mittelung wird weiterverarbeitet. Für die Messungen in dieser Arbeit wurde der Parameter

\footnotetext{
${ }^{1}$ Sechs Nachkommastellen auf der Anzeige, eine weitere interne Nachkommastelle kann über die Rechnerschnittstelle ausgelesen werden.
} 

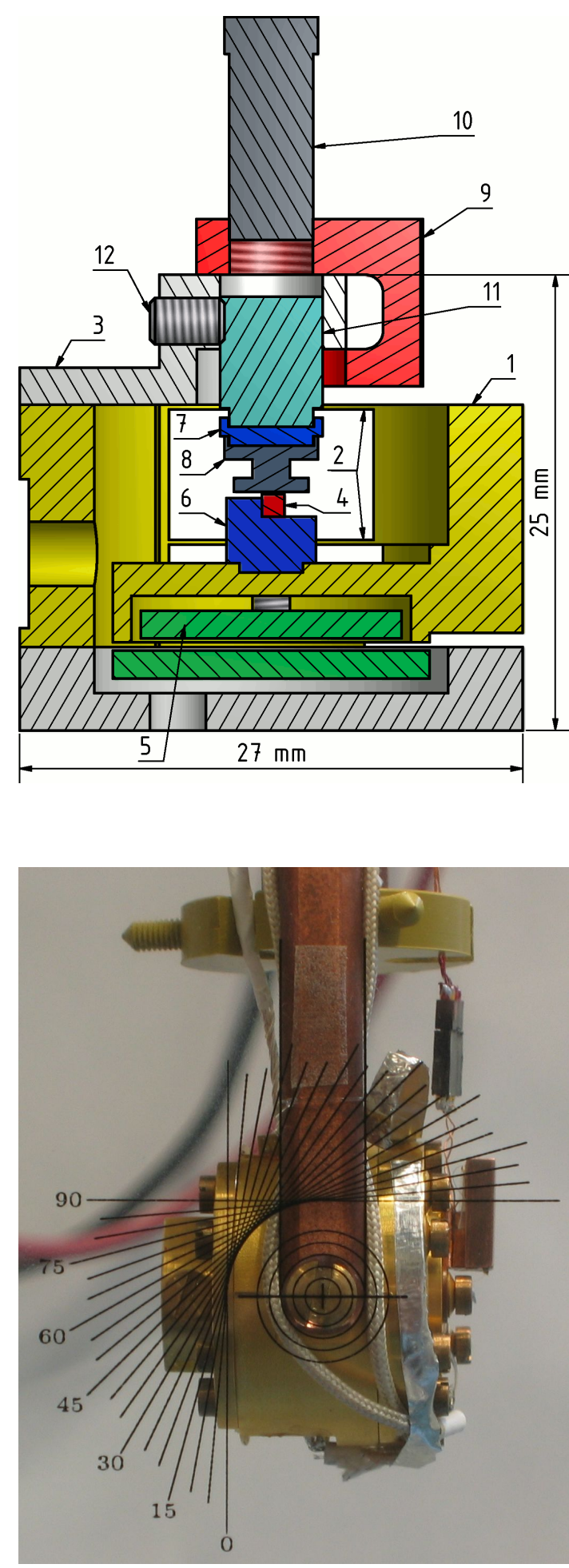

Abbildung 3.1: Schnitt durch das Dilatometer mit eingebauter, thermisch entkoppelter Probe. (1) beweglicher Rahmen, (2) Blattfedern, (3) fester Rahmen, (4) Probe, (5) Kondensatorplatten, (6) Probenhalter, (7) thermischer Isolator, (8) Silberstempel, (9) abnehmbare Spannvorrichtung mit (10) Spannschraube, (11) Spannstempel, (12) Klemmschraube.

Abbildung 3.2: Um $90^{\circ}$ gedrehtes Dilatometer beim Einbau. Das transparente Justiernetz ermöglicht die korrekte Ausrichtung der Meßzelle relativ zum vertikalen Halter. Die verstellbaren Torlonstifte (gelb, oben) zentrieren die Anordnung im Vakuumbecher. 
averexp auf den Wert 8 gesetzt, dies entspricht einer Mittelung über 64 Meßwerte im Abstand von $0,1 \mathrm{~s}$.

Die Verbindung der Kondensatorplatten zum Meßgerät erfolgt über je eine Koaxialleitung, für eine effiziente Schirmung des Signals müssen dabei die Außenleiter mit Masse verbunden werden. Um eine Erdschleife zu vermeiden, darf diese Verbindung aber nur an genau einem Punkt erfolgen. Aus diesem Grund wurden die BNC-Buchsen am Kopf des Kryostateneinsatzes sowie die SMB-Buchsen an der Mischungskammer mittels geeigneter Durchführungen aus Teflon elektrisch vollständig vom Kryostaten isoliert. Mit dem Dilatometer selbst sind die Außenleiter ebenfalls bewußt nicht verbunden. Die Schirme der Signalleitungen haben damit nur noch über ihren Anschluß an die Meßbrücke Massekontakt. Mit diesen Maßnahmen konnte das Rauschen um einen Faktor 10 reduziert werden und liegt im Bereich von $1 \cdot 10^{-6} \mathrm{pF}$ p-p. ${ }^{2}$

Bei einer Arbeitskapazität von ca. $25 \mathrm{pF}$ entspricht dies nach (3.4) (s.u.) einem Rauschen in der Länge von $2,5 \cdot 10^{-2} \AA$. Es läßt sich also festhalten, daß die Auflösung der Meßapparatur für Längenänderungen deutlich besser ist als $0,1 \AA$.

\subsubsection{Berechnung der Längenänderung}

Es gilt für die Kapazität eines einfachen Plattenkondensators:

$$
C=\epsilon_{\mathrm{r}} \epsilon_{0} \frac{A}{d}
$$

mit der elektrischen Feldkonstante $\epsilon_{0}=8,8542 \mathrm{Fm}^{-1}$, der relativen Permittivität $\epsilon_{\mathrm{r}}$ des Mediums zwischen den Kondensatorplatten, der Plattenfläche ${ }^{3} A=\pi r^{2}$ bei einem Plattenradius $r$ sowie dem Plattenabstand $d$. Dabei wird angenommen, daß das Verhältnis $r / d$ groß ist, so daß Randeffekte vernachlässigt werden können.

Das Dilatometer wird im Vakuum betrieben, so daß $\epsilon_{\mathrm{r}}=1$. Da $\epsilon_{\mathrm{r}, \mathrm{Luft}} \approx 1,0006$, kann auch für Tests im ausgebauten Zustand mit dem gleichen Zusammenhang gerechnet werden. Ändert sich die Kapazität gegenüber einem Startwert $C_{0}$ auf den Wert $C$, so ergibt sich eine Längenänderung

$$
\Delta l(C)=-\left(d(C)-d\left(C_{0}\right)\right)=\epsilon_{0} \pi r^{2} \frac{C-C_{0}}{C \cdot C_{0}} .
$$

Das Minuszeichen ensteht, da eine Ausdehnung der Probe eine Verringerung des Plattenabstands zur Folge hat.

Eine wichtige Fehlerquelle stellen nichtparallele Kondensatorplatten dar. Werden die Platten bei einem festen mittleren Abstand $d$ gegeneinander verkippt, so erhöht sich aufgrund der Nichtlinearität von (3.1) die gemessenen Kapazität und man errechnet nach (3.2) eine größere als die reale Längenänderung. Ein Maß für die Verkippung ist

\footnotetext{
${ }^{2}$ p-p: peak-to-peak (doppelter Scheitelwert)

${ }^{3}$ Die thermische Ausdehnung von $\mathrm{Cu}$ Hah70 verursacht zwischen Raum- und Heliumtemperatur eine Änderung der Plattenfläche von $\approx 3 \cdot 10^{-3}$. Dies liegt in der Größenordnung der Fertigungstoleranzen und wird deshalb vernachlässigt.
} 


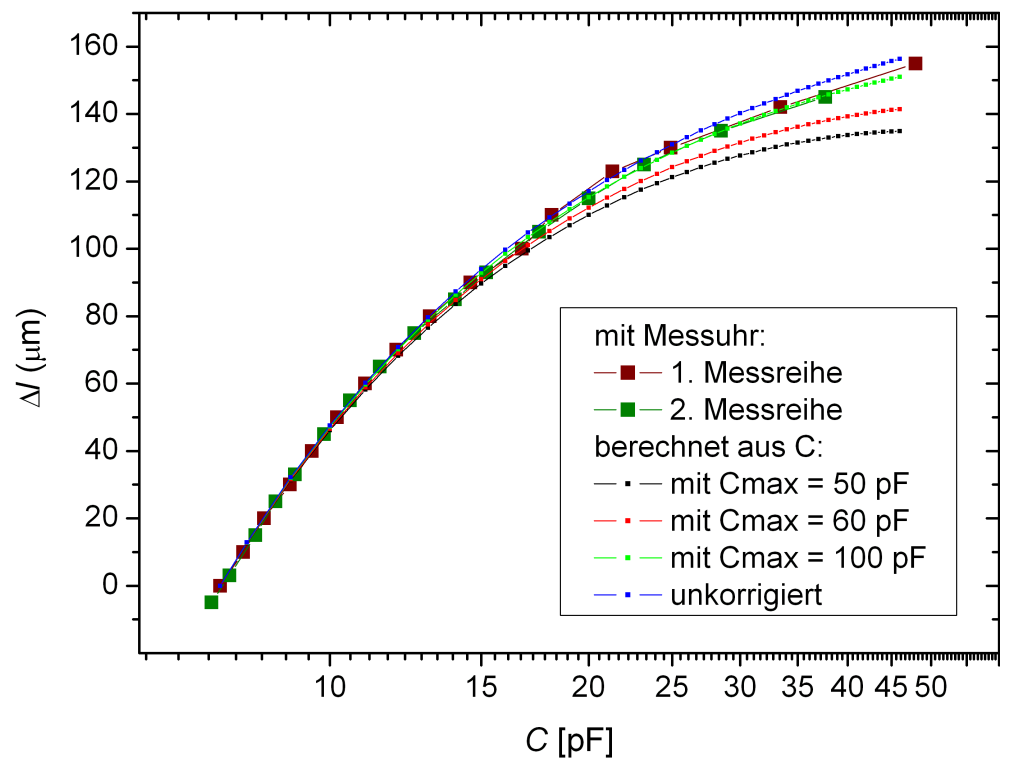

Abbildung 3.3: Funktionstest des Dilatometers bei Raumtemperatur. Die Längenänderung $\Delta l$ wurde mit einer Meßuhr in Schritten von 1/100 mm bestimmt bzw. nach (3.4) aus der gemessenen Kapazität mit Korrekturen für unterschiedliche Kurzschlußkapazitäten berechnet.

die Kurzschlußkapazität $C_{\max }$ : Während $C$ für den idealen Kondensator beliebig groß wird, ist $C_{\max }$ bei nicht völlig parallelen Platten endlich und geht als Parameter in die Korrektur ein, die von PotT und Schefzyk [PS83] zu

$$
\Delta l_{\mathrm{korr}}=\left(1-\frac{C \cdot C_{0}}{C_{\max }^{2}}\right) \Delta l
$$

bestimmt wird. Obwohl nach (3.1) die Empfindlichkeit $|\mathrm{d} C / \mathrm{d} d|_{d=d_{0}}=C / d_{0}$ proportional zur Kapazität ist, sollte also nach (3.3) die Arbeitskapazität $C_{0}$ nicht zu nahe bei $C_{\max }$ gewählt werden, damit der Einfluß der Plattenverkippung klein bleibt.

Die im Experiment gemessene Kapazität beträgt typischerweise einige zehn Picofarad und die Längenänderung $\Delta l$ wird bei tiefen Temperaturen gewöhnlich in Einheiten von $10^{-6} \mathrm{~cm}$ angegeben. Mit $r=7 \mathrm{~mm}$ erhält man

$$
\Delta l_{\text {korr }}\left[10^{-6} \mathrm{~cm}\right]=1,3898 \cdot 10^{5} \mathrm{pF} \cdot\left(1-\frac{C \cdot C_{0}}{C_{\max }^{2}}\right) \frac{C-C_{0}}{C \cdot C_{0}} .
$$

\subsubsection{Funktionstest}

Es ist hilfreich, die korrekte Funktion des Dilatometers und die Gültigkeit der Beziehung (3.4) zunächst in einem einfachen Versuch bei Raumtemperatur zu überprüfen. Für die Längenmessung wird dazu eine handelsübliche Meßuhr mit einer Auflösung von 1/100 mm verwendet, wie man sie gewöhnlich für feinmechanische Anwendungen einsetzt. Ihr Taster wird auf der Oberseite des beweglichen Rahmenteils angesetzt und 

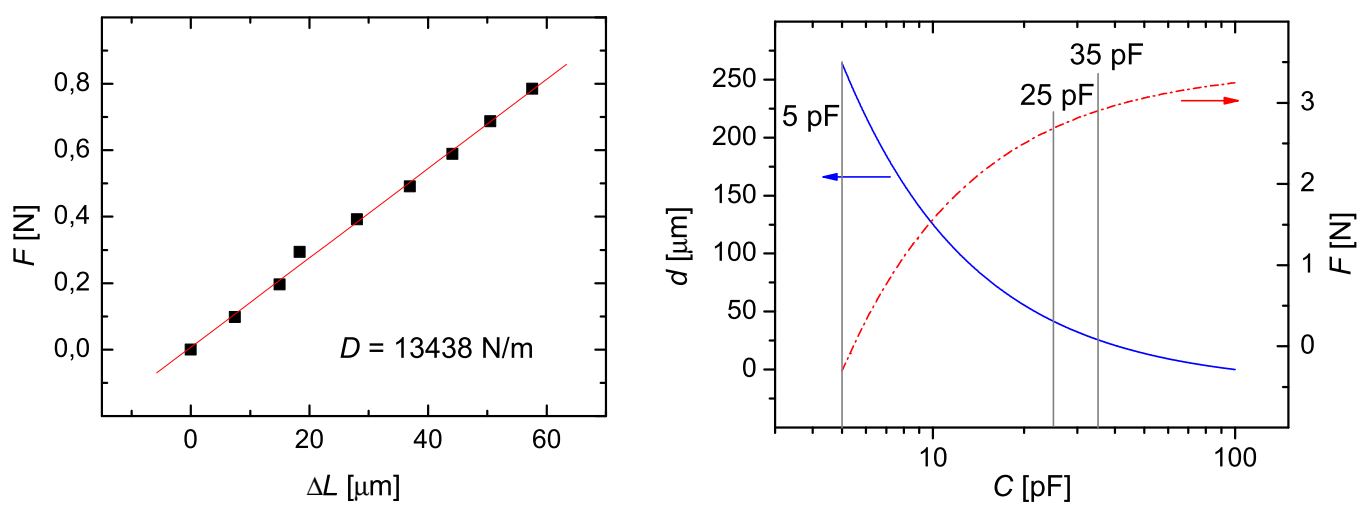

Abbildung 3.4: Links: Bestimmung der Federkonstante des Dilatometers durch Auflegen von Gewichten. Rechts: Bei typischen Arbeitskapazitäten wirkt eine Kraft von etwa $3 \mathrm{~N}$ auf die Probe.

die Wegänderung beim Anziehen der Spannschraube gemessen. Die Kapazität wird gleichzeitig auf der direkt angeschlossenen Meßbrücke beobachtet.

Abbildung 3.3 zeigt einen Vergleich der direkt gemessenen Längenänderung mit der aus der Kapazität berechneten. Die direkt gemessenen Längen stimmen gut mit den für $C_{\max }=100 \mathrm{pF}$ berechneten überein. Zwar muß die Ortsauflösung der Meßuhr im Vergleich zu den später zu messenden Längenänderungen als sehr grob beurteilt werden, das Experiment zeigt aber, daß die Längenänderung auf ca. 1\% korrekt ist. Die Maximalkapazität kann näherungsweise überprüft werden, indem die Spannschraube vorsichtig solange angezogen wird, bis sich ein Kurzschluß einstellt, und die höchste davor erreichte Kapazität notiert wird. Dieses Verfahren ist in gewisser Hinsicht willkürlich, da der Plattenabstand nur mit begrenzter Genauigkeit variiert werden kann, für die Messung von $C_{\max }$ aber ein infinitesimaler minimaler Plattenabstand eingestellt werden muß. Es zeigt sich aber zumindest, daß $C_{\max }=100 \mathrm{pF}$ einen vernünftigen Wert darstellt. Im Interesse der Reproduzierbarkeit werden deshalb alle Auswertungen mit diesem Wert durchgeführt, auch wenn die experimentell ermittelte Kurzschlußkapazität im Einzelfall leicht abweicht.

Der Funktionstest zeigt, daß die später vorgenommenen Messungen nicht nur hochpräzise sind in dem Sinne, daß kleine Längenänderungen aufgelöst werden können, sondern auch auf mindestens $2 \%$ genau.

\subsubsection{Druck auf die Probe}

Eine nicht zu vernachlässigende Konsequenz der oben beschriebenen Konstruktion des Dilatometers besteht in der Kraft, welche die Blattfedern notwendigerweise auf die eingebaute Probe ausüben. Um die Größe dieser Kraft abzuschätzen, wird zunächst die Meßzelle mit verschiedenen Gewichten bis $100 \mathrm{~g}$ belastet, um so, wie in Abbildung 3.4 (links) gezeigt, die Federkonstante $D$ der Blattfedern zu bestimmen. Da der 

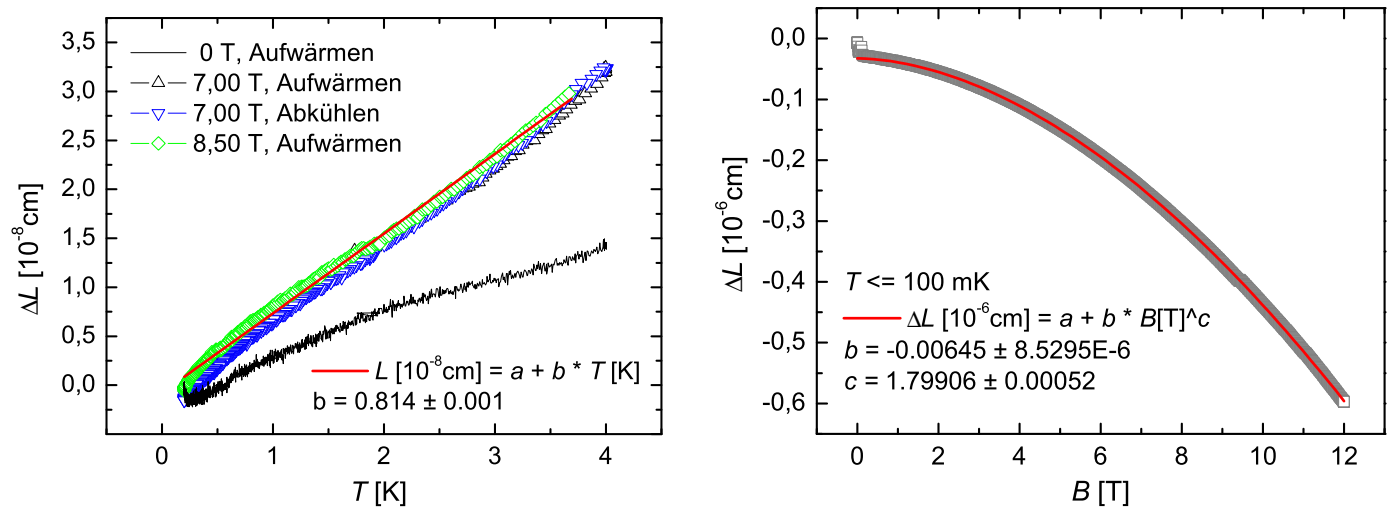

Abbildung 3.5: Leermessung der thermischen Ausdehnung (links) und Magnetostriktion (rechts) für das gedreht $(\Delta L \perp B)$ eingebaute Dilatometer. Beide Hintergründe sind ca. 1-2 Größenordnungen kleiner als die später gemessenen Effekte und werden bei der Datenanalyse abgezogen.

Elastizitätsmodul von Kupferlegierungen sich zu tiefen Temperaturen hin nur schwach ändert Cop RM67, kann der erhaltene Wert von $D=13438 \mathrm{Nm}^{-1}$ auch für den späteren Betrieb im Kryostaten angenommen werden.

Damit läßt sich nun der in Abbildung 3.4 (rechts) aufgetragene Zusammenhang zwischen Kapazität und Kraft bzw. Plattenabstand $d$ bestimmen. Im unbelasteten $\mathrm{Zu}$ stand ist $d \approx 265 \mu \mathrm{m}$. Um eine Arbeitskapazität $C_{0}$ zwischen $20 \mathrm{pF}$ und $35 \mathrm{pF}$ und damit eine hinreichende Empfindlichkeit zu erreichen, muß $d$ bis auf etwa $40 \mu \mathrm{m}$ verringert werden. Da nun $C$ empfindlich von $d$ abhängt, ist die letztlich anliegende Kraft vom konkret gewählten $C_{0}$ nahezu unabhängig. Sie ist vielmehr eine Funktion der Federkonstante und des anfänglichen Plattenabstands. Wie in Abbildung 3.4 (rechts) ersichtlich, ist im Experiment also mit einer auf die Probe wirkenden Kraft von knapp $3 \mathrm{~N}$ zu rechnen.

\subsubsection{Messung des Hintergrunds}

Im verwendeten Dilatometer kommen für den Rahmen und die Bauteile im Bereich der Probe unterschiedliche Werkstoffe zum Einsatz, so daß zu erwarten ist, daß Hintergrundeffekte nicht vollständig kompensiert werden. Daher wurden Leermessungen von thermischer Ausdehnung und Magnetostriktion in den später betrachteten Temperaturund Feldbereichen durchgeführt. Das Dilatometer wurde dazu um 90 Grad gedreht in der auch für die späteren Messungen verwendeten Konfiguration $\Delta L \perp B$ eingebaut. Außer einer Probe wurden alle in Abbildung 3.1 gezeigten Bauteile mitgemessen.

Die thermische Ausdehnung des leeren Dilatometers ist in Abbildung 3.5 (links) einmal für das Nullfeld und zusätzlich für die im Fall von $\mathrm{Sr}_{3} \mathrm{Ru}_{2} \mathrm{O}_{7}$ relevanten Magnetfelder von 7-8,5 T aufgetragen. Die Längenänderung unterscheidet sich zwischen $0 \mathrm{~T}$ und $7 \mathrm{~T}$ deutlich, variiert aber zwischen 7 und 8,5 T fast nicht. Außerdem ist dort 
$\Delta L$ näherungsweise linear in $T$; in Ermangelung eines konkreten Modells wird deshalb eine Gerade angepaßt. Mit einer Steigung von ca. $1 \cdot 10^{-8} \mathrm{cmK}^{-1}$ ist der Hintergrundeffekt eine bis zwei Größenordnungen kleiner als die in dieser Arbeit vermessenen Effekte, vgl. Abschnitt 5 .

Wie in Abschnitt 2.4 besprochen, erwartet man für ein einfaches Metall eine quadratische Magnetostriktion. Abbildung 3.5 (rechts) zeigt, daß sich $\Delta L(B)$ in der Tat gut durch ein Potenzgesetz mit einem Exponenten nahe 2 beschreiben läßt. Im Bereich um $8 \mathrm{~T}$ liegt dieser Hintergrundeffekt bei ca. $0,1 \cdot 10^{-6} \mathrm{cmT}^{-1}$ und damit ebenfalls eine bis zwei Größenordnungen unter der typischen Magnetostriktion von $\mathrm{Sr}_{3} \mathrm{Ru}_{2} \mathrm{O}_{7}$.

Aus den Kurvenanpassungen ergeben sich die Hintergründe als:

$$
\begin{aligned}
& \Delta L^{\mathrm{HG}}(T)=8,1 \cdot 10^{-9} \mathrm{~cm} \cdot T[\mathrm{~K}] \\
& \Delta L^{\mathrm{HG}}(B)=6,5 \cdot 10^{-9} \mathrm{~cm} \cdot(B[\mathrm{~T}])^{1,8}
\end{aligned}
$$

Sie sind bei allen in dieser Arbeit gezeigten Daten abgezogen.

\subsection{Numerische Differentiation}

In dieser Arbeit sind Größen von Interesse, die die Ableitung einer gemessenen Größe darstellen. Beispiele hierfür sind die thermische Ausdehnung $\alpha$, die Magnetostriktion $\lambda$ oder auch die spezifische Wärme $c$. Die experimentell bestimmte Meßgröße $y(x)$ (also z.B. $\Delta L(T))$ liegt dabei in Form von Wertepaaren $\left(x_{i}, y_{i}\right)$ vor. ${ }^{4}$ Es stellt sich nun die Aufgabe, aus diesen Meßwerten auf numerischem Wege die Ableitung $\mathrm{d} y(x) / \mathrm{d} x \mathrm{zu}$ bestimmen.

Das einfachste Verfahren hierfür ist die Berechnung des Differenzenquotienten $\left(y_{i}-\right.$ $\left.y_{i-1}\right) /\left(x_{i}-x_{i-1}\right)$. Reale Meßdaten sind allerdings immer mit einem gewissen Rauschen behaftet, welches lokal einen sehr starken Einfluß auf den Differenzenquotienten hat und die Methode deshalb ungeeignet macht. Es ist also ein Verfahren gesucht, welches die lokale Ableitung möglichst exakt bestimmt und gleichzeitig unempfindlich gegenüber Rauschen ist.

Ist die Funktion $y(x)$ analytisch bekannt, so ist eine Anpassung nach der Methode der kleinsten Fehlerquadrate ein geeignetes Verfahren. Für die in dieser Arbeit diskutierten Daten gibt es allerdings i. A. kein theoretisches Modell des Kurvenverlaufs. Deshalb kommen hier zwei andere Verfahren zum Einsatz.

\section{Lokale Bestimmung der Steigung auf einem Intervall mittels linearer Regression.} Die lokale Steigung $\mathrm{d} y(x) /\left.\mathrm{d} x\right|_{x=x 0}$ ergibt sich hierbei durch lineare Regression über die Menge $M=\left\{\left(x_{i}, y_{i}\right) \mid x_{i} \in\left[x_{0}-l / 2, x_{0}+l / 2\right]\right\}$, also die Datenpunkte, die in einem bei $x_{0}$ zentrierten Intervall der Länge $l$ liegen. Abbildung 3.6 zeigt dies für eine verrauschte Sinusfunktion. Das Verfahren soll also eine Kosinusfunktion möglichst gut reproduzieren, wobei die Intervallänge $l$ so groß gewählt werden muß, daß die errechnete

\footnotetext{
${ }^{4}$ Die $x_{i}$ seien oBdA monoton, also $x_{i}>x_{j} \forall i>j$.
} 


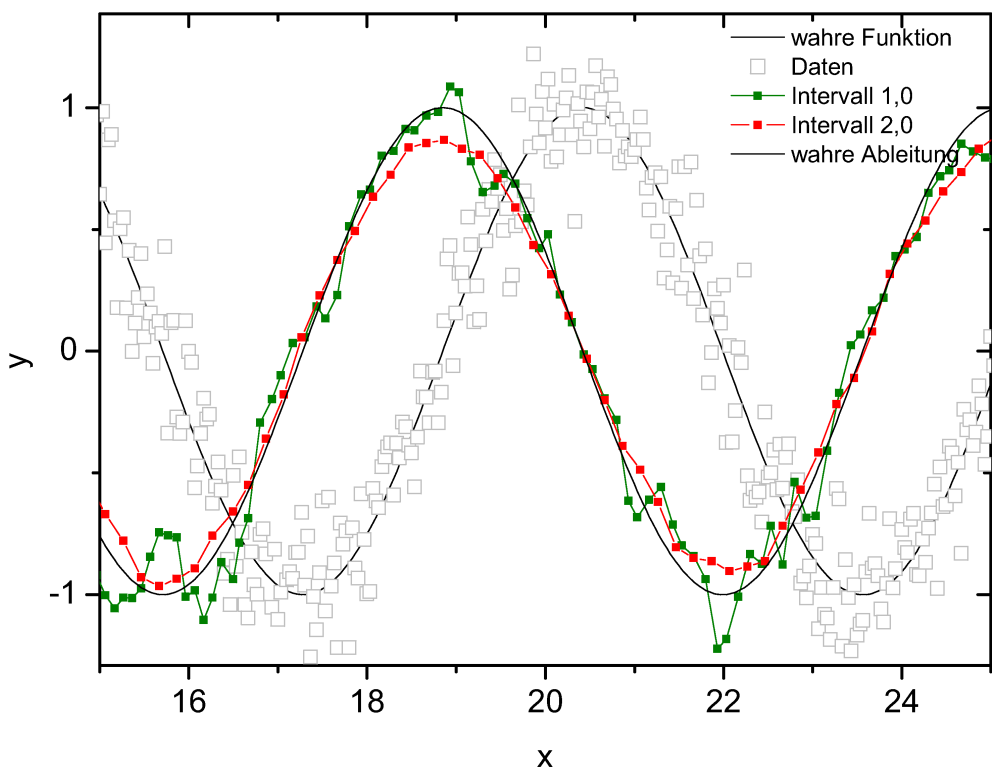

Abbildung 3.6: Numerische Differentiation von rauschbehafteten Meßdaten. Simulierte Daten $y_{i}=\sin \left(x_{i}\right)+r_{i}$. Das Rauschen $r$ ist eine normalverteilte Zufallsvariable mit $\sigma=0,2$. Farbige Kurven: Bestimmung der Ableitung durch lokale lineare Regression über verschiedene Intervalle.

Ableitung hinreichend glatt erscheint. In diesem Beispiel zeigt die Kurve für $l=1,0$ ein deutliches Rauschen, was sich durch ein größeres Glättungsintervall $l=2,0$ verbessern läßt. Nun zeigt sich aber, daß die extremalen Werte $\mathrm{d} y / \mathrm{d} x=1,-1$ nicht mehr erreicht werden: die Extrema der Ableitung werden durch die Glättung ausgeschmiert.

Anpassung eines kubischen Splines Eine alternative Methode besteht in der Anpassung eines kubischen Splines an die Meßdaten. Dazu wird der Definitionsbereich in $n$ Intervalle aufgeteilt, auf denen die Funktion stückweise durch Polynome dritten Grades beschrieben wird. Die Anschlußbedingungen an den Knotenpunkten werden so gewählt, daß Funktionswert sowie erste und zweite Ableitung übereinstimmen. Die entstehende Funktion ist dadurch zweimal stetig differenzierbar. Diese Eigenschaft läßt sich ausnutzen, um eine glatte Ableitung der durch einen Spline beschriebenen Meßdaten zu erhalten. Für eine vertiefte Diskussion sei auf das Lehrbuch [Boo78] verwiesen.

Es existieren Algorithmen, die zu einer gegebenen Datenmenge automatisch einen Glättungsspline $\operatorname{spl}(x)$ erzeugen. Hier wird eine in Nag beschriebene Funktion der Numerical Applications Group verwendet. Der Algorithmus wählt die Knoten des Splines so, daß die die Größe

$$
\sum_{i}\left\|\operatorname{spl}\left(x_{i}\right)-y_{i}\right\|^{2}+s \int\left\|\frac{\partial^{2} \operatorname{spl}(x)}{\partial x^{2}}\right\|^{2} \mathrm{~d} x
$$

minimal wird. Der erste Term beschreibt dabei die Abweichung des Splines von den 


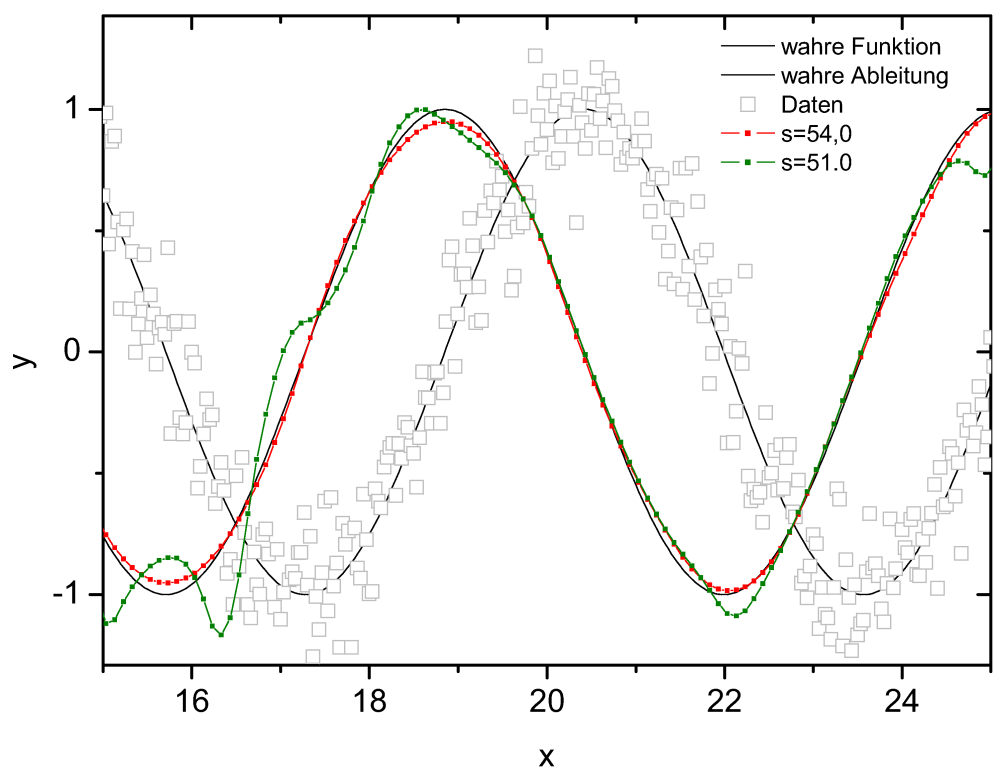

Abbildung 3.7: Numerische Differentiation von rauschbehafteten Meßdaten wie in Abbildung 3.6. Farbige Kurven: Ableitung des mit unterschiedlichen Glättungsparametern $s$ angepaßten Splines.

Datenpunkten. Der zweite, mit dem Glättungsfaktor $s$ gewichtete Term ist ein Maß für die Krümmung der Kurve. Für kleine $s$ liegt also der Spline sehr nahe an den Datenpunkten, ${ }^{5}$ was insbesondere in der Ableitung zu unerwünschten Oszillationen führen kann. Ein großes $s$ bevorzugt dagegen eine möglichst glatte Kurve, die aber möglicherweise stark von den Daten abweicht, so daß Details verloren gehen können. Der Parameter $s$ muß also empirisch so gewählt werden, daß sich ein guter Kompromiß zwischen Datentreue und Glättung ergibt.

Abbildung 3.7 demonstriert wiederum die Ableitung einer verrauschten Sinusfunktion: Der Glättungsfaktor wird empirisch solange erhöht, bis keine unerwünschten Überschwinger mehr auftreten. Die beste Anpassung ergibt sich hier für $s=54$.

Vergleich Eine Betrachtung der roten Kurven in den Abbildungen 3.6 und 3.7 zeigt, daß der Glättungsspline die wahre Ableitung etwas besser approximiert und dabei konstruktionsbedingt glatt ist, während die aus der Intervallableitung erhaltenen Daten besonders im Bereich der Extrema stärker abweichen und zusätzlich ein Rauschen aufweisen. Für die Auswahl des zu verwendenden Verfahrens sind aber noch weitere Aspekte wesentlich:

Bei der Intervallableitung handelt es sich um eine lokale Methode: Die Ableitung $\mathrm{d} y(x) /\left.\mathrm{d} x\right|_{x=x 0}$ ist nur von den Datenpunkten in unmittelbarer Umgebung von $x_{0}$ abhängig. Der Spline ist dagegen das Ergebnis einer globalen Optimierung, die das

\footnotetext{
${ }^{5}$ Für $s=0$ erhält man einen interpolierenden Spline, der durch alle Datenpunkte verläuft.
} 
Maß (3.7) minimiert. Deshalb ist der Wert der Ableitung an einer gegebenen Stelle letztlich eine Funktion aller Datenpunkte. Fehler an weiter entfernten Stellen der Kurve können also die lokale Ableitung verfälschen. Weiterhin ist das Rauschen in der Intervallableitung auch ein Maß für die Qualität der Ausgangsdaten, während der glatte Spline hierüber keine Rückschlüsse ermöglicht.

In dieser Arbeit wird deshalb im folgenden stets die Methode der Intervallableitung eingesetzt. 



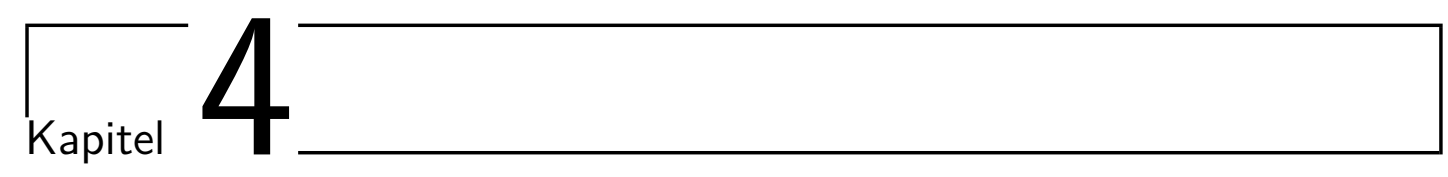

\section{Der itinerante Metamagnet $\mathrm{Sr}_{3} \mathrm{Ru}_{2} \mathrm{O}_{7}$}

Das System $\mathrm{Sr}_{3} \mathrm{Ru}_{2} \mathrm{O}_{7}$ hat in den letzten 10 Jahren eine beträchtliche Aufmerksamkeit in der experimentellen und theoretischen Festkörperphysik erhalten. In unmittelbarer Nähe eines quantenkritischen Endpunkts bei ca. $8 \mathrm{~T}$ bildet sich in $\mathrm{Sr}_{3} \mathrm{Ru}_{2} \mathrm{O}_{7}$ eine „nematische Phase" mit anisotropen elektronischen Transporteigenschaften, deren genaue Natur allerdings noch nicht abschließend geklärt ist. Im folgenden wird der bisherige Stand der Forschung zusammengefaßt.

\subsection{Kristallstruktur und elektronische Eigenschaften}

Bei $\mathrm{Sr}_{3} \mathrm{Ru}_{2} \mathrm{O}_{7}$ handelt es sich um ein Mitglied der Ruddlesden-Popper(RP)-Serie der Form $\mathrm{Sr}_{n+1} \mathrm{Ru}_{n} \mathrm{O}_{3 n+1}$, wobei der Parameter $n$ die Zahl der aufeinanderfolgenden RuOEbenen und damit eine effektive Dimensionalität beschreibt. Für unterschiedliche Werte von $n$ weist die Serie weitere interessante Vertreter auf. Während das Perowskit $\mathrm{SrRuO}_{3}(n=\infty)$ ein dreidimensionaler Ferromagnet mit einer Curietemperatur von $160 \mathrm{~K}$ ist All96], handelt es sich beim doppellagigen $\mathrm{Sr}_{2} \mathrm{RuO}_{4}$ um ein quasi zweidimensionales Metall, in dem bei $T_{\mathrm{c}} \approx 1,3 \mathrm{~K}$ unkonventionelle Supraleitung einsetzt (vgl. den Übersichtsartikel [MM03]). Im Gegensatz zu anderen unkonventionellen Supraleitern findet hier Spin-Triplett-Paarung statt, d.h. zwei Elektronen mit parallelem Spin bilden ein Cooperpaar mit $S=1$. Der Ordnungsparameter hat $p$-Wellen-Symmetrie und die Supraleitung tritt (im Gegensatz zu den Hochtemperatursupraleitern) bereits im undotierten System auf. Solche Ergebnisse motivierten die Suche nach weiteren interessanten Materialien in der RP-Serie.

Perowskite besitzen i. A. eine tetragonale Struktur mit der Raumgruppe I4mm; in $\mathrm{Sr}_{3} \mathrm{Ru}_{2} \mathrm{O}_{7}$ führt allerdings eine durch den verhältnismäßig großen Ionenradius von $\mathrm{Ru}$ hervorgerufene Druckspannung zu einer kooperative Rotation der RuO-Oktaeder um etwa $7^{\circ}$, welche, wie in Abbildung 4.1 gezeigt, die Symmetrie zu der der orthorhombischen Raumgruppe Bbcb erniedrigt [Sha00a]. In der $a b$-Ebene ergibt sich dadurch eine um den Faktor $\sqrt{2}$ vergrößerte Einheitszelle mit den Gitterkonstanten $a \approx b \approx 5,50 \AA$ 
a)

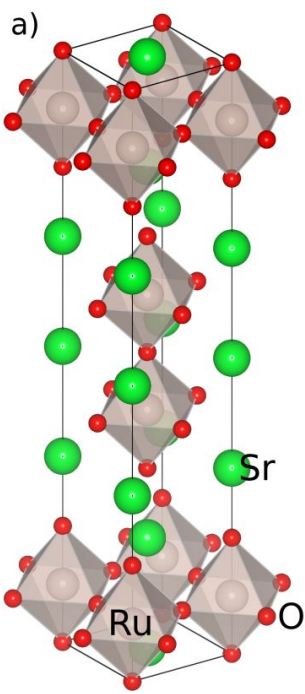

b)

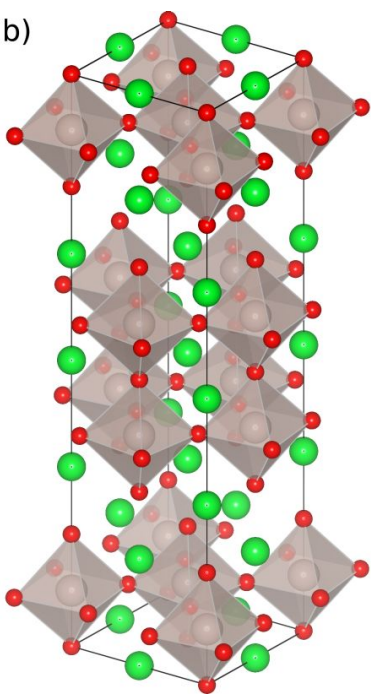

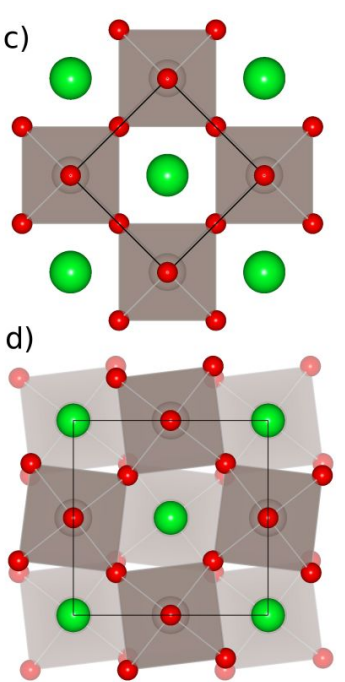

Abbildung 4.1: Kristallstruktur von $\mathrm{Sr}_{3} \mathrm{Ru}_{2} \mathrm{O}_{7}$. Jeweils zwei aufeinanderfolgende Lagen von $\mathrm{RuO}$ sind durch isolierende Lagen von $\mathrm{SrO}$ voneinander getrennt. Durch kooperative Rotation der RuO-Oktaeder um $7^{\circ}$ vergrößert sich die tetragonale Einheitszelle (a und c) zu einer orthorhombischen (b und d). Visualisiert mit VESTA [MI08.

und $c \approx 20,72 \AA$ bei Raumtemperatur Sha00b. Obwohl die Gitterparameter $a$ und $b$ praktisch gleich sind, gibt es in der Ebene keine vierfache Rotationssymmetrie mehr. Die Kristallstruktur wird deshalb auch als pseudotetragonal bezeichnet. In dieser Arbeit beziehen sich kristallographische Achsen $(a, b, c)$ stets auf das genannte pseudotetragonale System.

Die elektronische Struktur von $\mathrm{Sr}_{3} \mathrm{Ru}_{2} \mathrm{O}_{7}$ ist relativ kompliziert, da viele Bänder das Ferminiveau schneiden. In einer Tight-Binding-Näherung läßt sich die Fermifläche allerdings mit heuristischen Argumenten konstruieren [Mer10], wobei die Bänder aus den $t_{2 g^{-}}$-Orbitalen der Ru-4d-Elektronen hervorgehen. Die $d_{x z^{-}}$bzw. $d_{y z}$-Bänder haben quasi-eindimensionalen Charakter, da der orbitale Überlapp jeweils nur ein Hüpfen in der $x$ - bzw. $y$-Richtung ermöglicht und der Transport in $z$-Richtung durch die SrLagen unterbunden wird. Das $d_{x y}$-Band ist dagegen zweidimensional. Durch die Doppellagenaufspaltung findet man jedes dieser Bänder zweimal. Die Vergrößerung der Einheitszelle durch die Oktaederrotation führt zu einer entsprechend kleineren Brillouinzone und im reduzierten Zonenschema kommt es zu einer Rückprojektion in die erste Brillouinzone.

Dort, wo sich verschiedene Bänder schneiden, bilden sich durch Hybridisierung Energielücken aus und es entsteht eine komplexe Fermifläche mit mehreren Teilfächen. Mittels winkelaufgelöster Photoemissionsspektroskopie (angle-resolved photoemission spectroscopy, ARPES) läßt sich diese mit hoher Genauigkeit vermessen. Mit dieser Methode erhielten TAMAI et al. Tam08 die in Abbildung 4.2 wiedergegebene Bandstruktur, die eine gute Übereinstimmung mit Bandstrukturrechnungen aufweist. Ein 


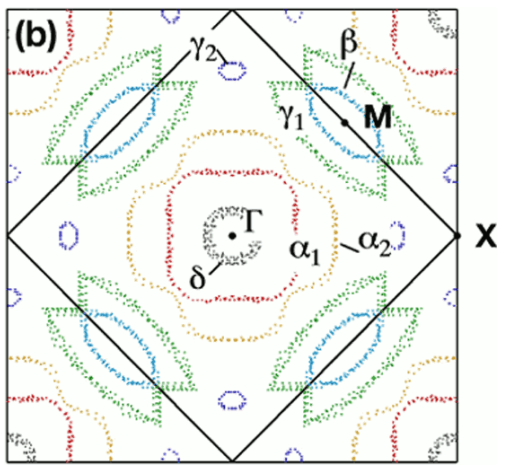

Abbildung 4.2: Die Fermifläche von $\mathrm{Sr}_{3} \mathrm{Ru}_{2} \mathrm{O}_{7}$, extrahiert aus ARPES-Messungen [Tam08]. Die Brillouinzone halbiert sich durch die Oktaederrotation auf die Fläche der inneren Raute.

Vergleich mit diesen Rechnungen ermöglicht außerdem die Zuordnung der Fermiteilflächen zu den atomaren Orbitalen.

ARPES-Messungen geben auch Hinweise auf die Existenz einer scharfen Spitze in der Zustandsdichte. Diese van-Hove-Singularität liegt ca. 3,5 eV unter der Fermikante beim X-Punkt in der Nähe der $\gamma_{2}$-Fermiteilfäche und spielt wahrscheinlich eine wichtige Rolle für den weiter unten diskutierten Metamagnetismus.

Im gesamten Bereich unterhalb von Raumtemperatur ist das Transportverhalten metallisch, wobei der spezifische Widerstand $\varrho$ in Richtung der $c$-Achse um einen Faktor 40 bis 300 größer ist als parallel zu den RuO-Ebenen. Dies spiegelt den stark zweidimensionalen Charakter des Systems wider, der bereits in der obigen Diskussion von Kristallstruktur und Fermiflächen deutlich wurde. Für tiefe Temperaturen verhält sich der spezifische Widerstand wie $\varrho=\varrho_{0}+A T^{2}$, was $\mathrm{Sr}_{3} \mathrm{Ru}_{2} \mathrm{O}_{7}$ als eine Fermiflüssigkeit ausweist Ike00. Der Sommerfeldkoeffizient $\gamma$ ist mit $110 \mathrm{~mJ} /\left(\mathrm{mol} \mathrm{Ru} \mathrm{K}^{2}\right)$ gegenüber anderen System leicht erhöht, die effektiven Massen der Ladungsträger [Mer09] liegen im Bereich von $m^{*}=5 . .20 m_{\mathrm{e}}$. Dies klassifiziert $\mathrm{Sr}_{3} \mathrm{Ru}_{2} \mathrm{O}_{7}$ noch nicht als SchweresFermionen-, aber doch als ein starkkorreliertes Elektronensystem. Suszeptibilitätsmessungen [Ike00] zeigen ein paramagnetisches Curie-Weiss-Verhalten ohne spontane magnetische Ordnung, doch ist $\chi$ im Grundzustand stark austauschverstärkt. Zusammen mit einem Wilsonverhältnis (2.4) von etwa 10 deutet dies auf eine Nähe zu einer ferromagnetischen Instabilität hin. In der Tat läßt sich durch einen verhältnismäßig geringen uniaxialen Druck in der $c$-Richtung von etwa 4 kbar Ferromagnetismus induzieren [YPM06].

\subsection{Metamagnetismus und Quantenkritikalität}

In itineranten Ferromagneten kommt es unter Einfluß der Austauschwechselwirkung zu einer spontanen Magnetisierung der Leitungselektronen. Eine erhöhte kinetische Energie des Gesamtsystems wird dabei durch eine Absenkung der Coulombenergie überkompensiert. Bedingung für das Auftreten einer solchen spontanen Spinaufspaltung ist das bekannte Stoner-Kriterium Blu01:

$$
U D\left(\epsilon_{\mathrm{F}}\right) \geq 1
$$


Dabei ist $U$ ein Maß für die Stärke der Austauschwechselwirkung und $D\left(\epsilon_{\mathrm{F}}\right)$ die $\mathrm{Zu}$ standsdichte bei der Fermienergie. In den klassischen Bandferromagneten Co, Fe und Ni liegt $\epsilon_{\mathrm{F}}$ in einem schmalen 3d-Band, wodurch Gleichung (4.1) erfüllt wird.

Weiterhin gibt es nun Fälle, in denen die Fermienergie zu weit von einer Singularität in der Zustandsdichte entfernt ist, um dem Stonerkriterium zu genügen. Im Nullfeld ist das System damit paramagnetisch. Wie in Abbildung 4.3 dargestellt, kann ein äußeres Magnetfeld aber das chemische Potential so verschieben, daß die Singularität wieder in der Nähe der Fermikante zu liegen kommt und damit für den Stonermechanismus zugänglich wird. ${ }^{1}$ Zusätzlich zum linearen paramagnetischen Verhalten der Magnetisierung kommt es zu einer stärkeren, spontanen Magnetisierungszunahme. Dieser Effekt wird als Metamagnetismus bezeichnet.

In $\mathrm{Sr}_{3} \mathrm{Ru}_{2} \mathrm{O}_{7}$ treten metamagnetische Übergänge sowohl für Felder in der als auch senkrecht zur ab-Ebene auf. Für $B \| a b$ beträgt das metamagnetische kritische Feld $B_{\mathrm{c}}^{\mathrm{mm}}$ etwa $5,5 \mathrm{~T}$, für $B \| c$ findet man $B_{\mathrm{c}}^{\mathrm{mm}} \approx 7,8 \mathrm{~T}$ [Per01]. Messungen der komplexen magnetischen Wechselfeldsuszeptibilität $\chi$ zeigen unterhalb einer kritischen Tempera$\operatorname{tur} T^{*}$ einen nichtverschwindenden Imaginärteil und damit Hystereseeffekte Gri03, was auf einen Übergang erster Ordnung hindeutet. In der $B$ - $T$-Ebene findet man eine Linie von Übergängen erster Ordnung, die in einem kritischen Endpunkt $\left(T^{*}, H^{*}\right)$ endet. Wird nun das Magnetfeld kontinuierlich aus der ab-Ebene in Richtung der cAchse verkippt, so wird eine Erhöhung von $B_{\mathrm{c}}^{\mathrm{mm}}$ beobachtet, einhergehend mit einer Abnahme der kritischen Temperatur $T^{*}$. Für $\Theta=\Varangle(B, a b) \rightarrow 90^{\circ}$ geht $T^{*}$ schließlich gegen 0 , vgl. Abbildung 4.4. In der $(T, \Theta)$-Ebene erhält man so eine Linie von kritischen Endpunkten, die für $\Theta \rightarrow 90^{\circ}$ zu $T=0$ unterdrückt wird. Aufgrund dieser Situation spricht man auch von einem quantenkritischen Endpunkt (QKEP).

Dieses Bild wird weiter unterstützt durch Untersuchungen der Transporteigenschaften im Bereich um den QKEP. Während die Temperaturabhängigkeit des spezifischen Widerstands $\varrho=\varrho_{0}+A T^{\alpha}$ im Nullfeld mit $\alpha=2$ FF-Verhalten aufweist, beobachtet man in einem charakteristischen „Trichter" über dem QKEP einen anomalen Exponenten von $\alpha<2$, vgl. Abbildung 4.5. Außerdem treten bei Annäherung an den QKEP Divergenzen sowohl im Koeffizienten $A$ Gri01 als auch in der spezifischen Wärme [Per01] auf.

Ein quantenkritischer Punkt im herkömmlichen Sinne entsteht aus der Unterdrückung eines Übergangs zweiter Ordnung (siehe Abschnitt 2.3 und trennt daher im Phasendiagramm zwei Bereiche unterschiedlicher Symmetrie, bspw. einen Antiferromagneten von einem Paramagneten. Im hier vorliegenden Fall entsteht der QKEP dagegen aus der Unterdrückung einer Linie von kritischen Endpunkten und trennt zwei Grundzustände, die sich lediglich durch den Grad der Spinpolarisation unterscheiden. Der QKEP ist deshalb von einem Bereich konstanter Symmetrie umgeben. Die experimentellen Ergebnisse zeigen also, daß eine spontane Symmetriebrechung keine notwendige Bedingung für das Auftreten von quantenkritischem Verhalten ist. Den Kontrollpara-

\footnotetext{
${ }^{1}$ Die Zeemanenergie $-\mu_{\mathrm{B}} B$ ist im Vergleich zu typischen Fermienergien und Bandbreiten von einigen eV gering; $\mu_{\mathrm{B}}=9 \cdot 10^{-24} \mathrm{~J} / \mathrm{T} \approx 0,1 \mathrm{meV} / \mathrm{T}$. $E_{\mathrm{F}}$ muß deshalb sehr nahe an der Singularität liegen.
} 

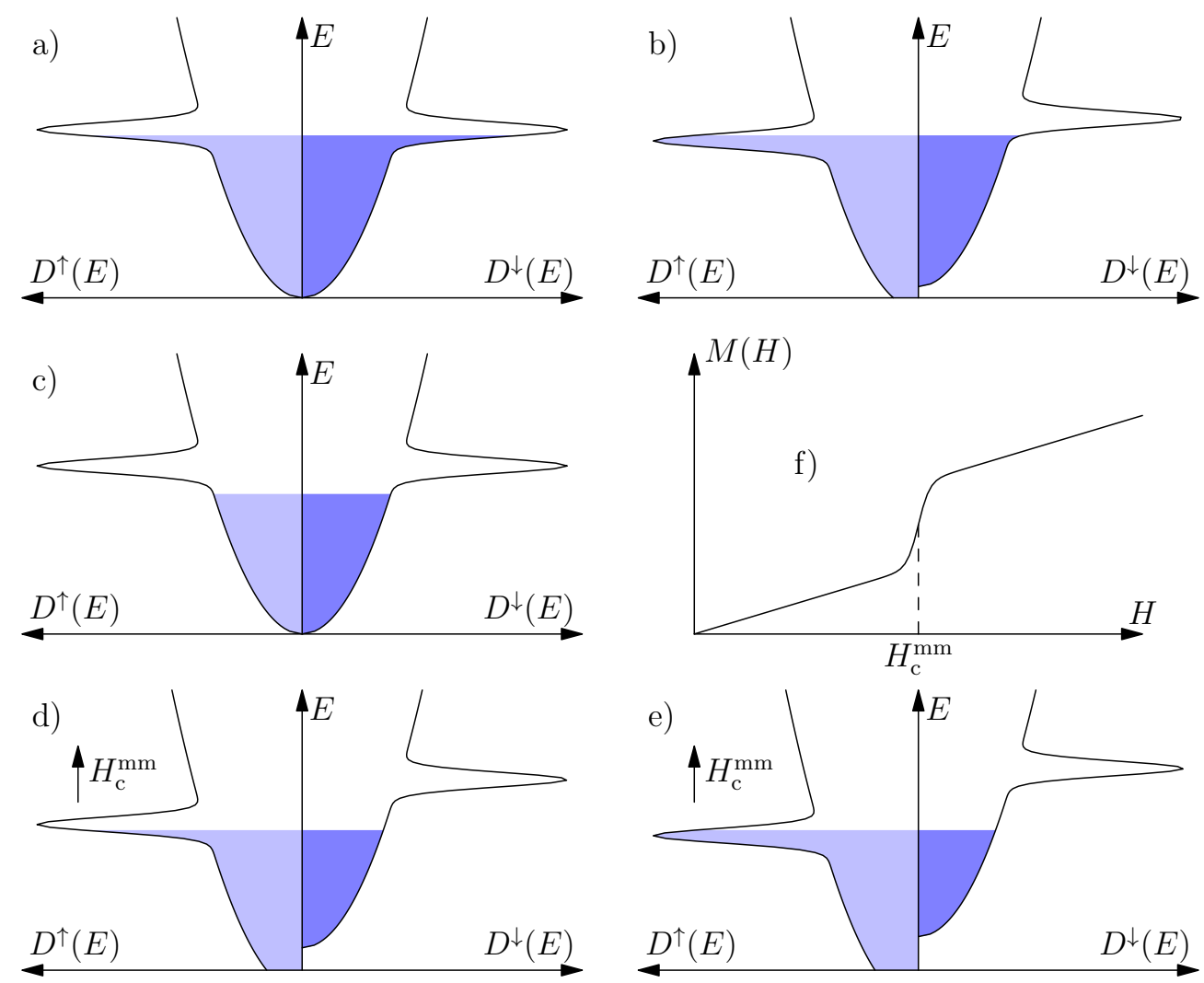

Abbildung 4.3: Illustration zum Metamagnetismus: In a) liegt die Fermienergie in der Nähe einer van-Hove-Singularität und das Stoner-Kriterium Gl. 4.1) ist erfüllt. Es bildet sich eine spontane Magnetisierung wie in b) aus. Dies ist der Fall eines Ferromagneten. Im Fall c) ist das Stoner-Kriterium gerade nicht erfüllt. Wird aber wie in d) zusätzlich ein äußeres metamagnetisches kritisches Magnetfeld $H_{\mathrm{c}}^{\mathrm{mm}}$ angelegt, so verschiebt die Zeeman-Aufspaltung die Fermienergie (zumindest für ein Subband) wieder in die Nähe der Singularität. Wie in e) gezeigt, kommt es nun zu einer spontanen Zunahme der Magnetisierung bei $H=H_{\mathrm{c}}^{\mathrm{mm}}$. Die typische Magnetisierungskurve $M(H)$ des Metamagneten ist in f) gezeigt. 


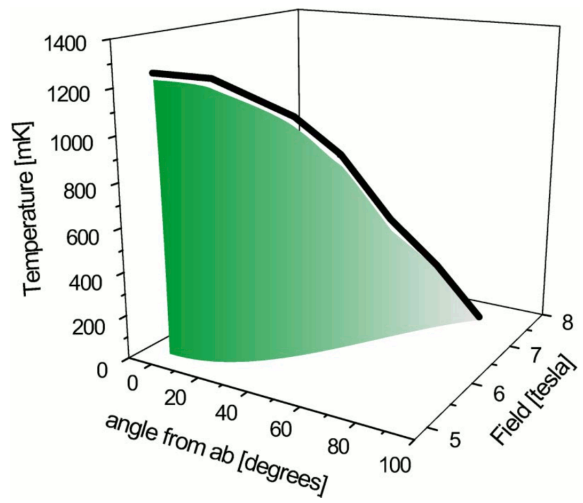

Abbildung 4.4: Durch Verkippen des Magnetfelds aus der $a b$-Ebene in die $c$-Richtung wird die kritische Temperatur $T^{*}$, die den Endpunkt (schwarz) einer Linie von metamagnetischen Übergängen erster Ordnung (grüne Fläche) darstellt, zu $T=0$ unterdrückt. Der Winkel $\Theta=\Varangle(B, a b)$ fungiert als Kontrollparameter, der das System für $\Theta=90^{\circ}$ auf den quantenkritischen Endpunkt einstellt. Aus Gri03.

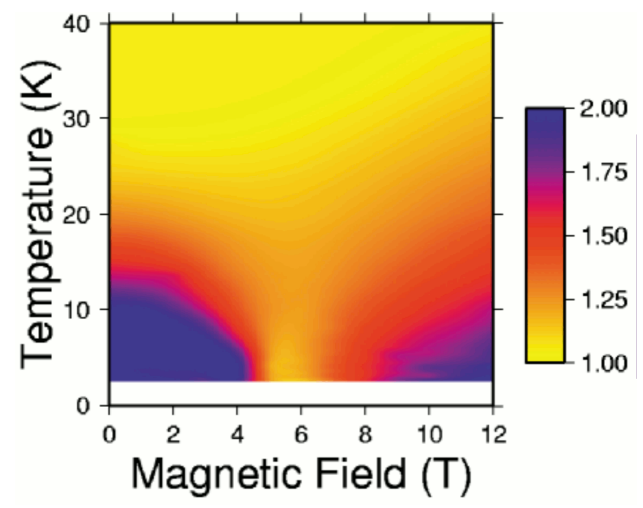

Abbildung 4.5: Exponent der Temperaturabhängigkeit des spezifischen Widerstands $\varrho=\varrho_{0}+A T^{\alpha}$ von $\mathrm{Sr}_{3} \mathrm{Ru}_{2} \mathrm{O}_{7}$ für Felder in der $a b$-Ebene. In einem trichterförmigen Bereich über dem QKEP tritt NFF-Verhalten mit $\alpha<2$ auf. Aus Per01].

meter ist durch den Winkel $\Theta$ gegeben.

\subsection{Magnetoelastische Kopplung und thermische Ausdehnung}

Messungen der Magnetostriktion $\lambda$ Geg06a; Gri04 ergeben eine Signatur, die in hohem Maße derjenigen der magnetischen Suszeptibilität $\chi=\mathrm{d} M / \mathrm{d} H$ ähnelt. Insbesondere lassen sich die metamagnetischen Übergänge im Bereich um $8 \mathrm{~T}$ sehr deutlich beobachten, was auf eine starke Kopplung der magnetischen Freiheitsgrade an das Gitter hindeutet. $\lambda$ mißt nach Gleichung (2.9) die Ableitung der Magnetisierung nach dem Druck $p$, während $\chi$ diejenige nach dem Magnetfeld darstellt. Die starke Ähnlichkeit der zwei Größen deutet also darauf hin, daß Ableitungen der freien Energie nach $p$ und $H$ dieselbe thermodynamische Information abbilden. Es bietet sich deshalb an, auch Größen wie $\alpha$ und $\lambda$ auf quantenkritische Effekte zu untersuchen.

Gegenwart et al. Geg06b haben die thermische Ausdehnung in $c$-Richtung gemessen und dabei das in Abbildung 4.6 (links) gezeigte Verhalten gefunden. Für tiefe Temperaturen zeigt sich FF-Verhalten mit $\alpha \propto T$, während $\alpha$ im quantenkritischen Bereich ein Maximum annimmt und danach wieder fällt. Mit Annäherung an das kritische Feld wird dieses Maximum schärfer und wandert zu niedrigeren Temperaturen. Beim kritischen Feld durchläuft $\alpha$ einen Vorzeichenwechsel und wird für $H>H_{\mathrm{c}}$ negativ.

Unter der Annahme, daß der Druck nur über die Kopplung an das metamagnetische 

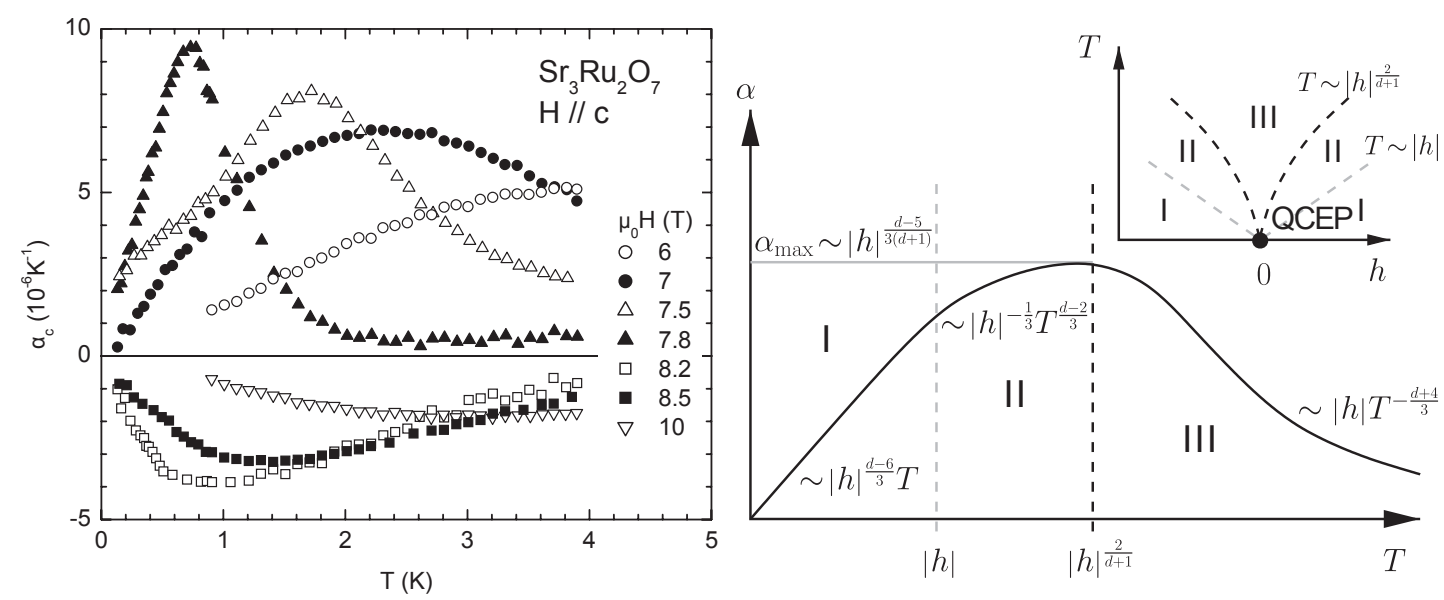

Abbildung 4.6: (links) Die thermische Ausdehnung von $\mathrm{Sr}_{3} \mathrm{Ru}_{2} \mathrm{O}_{7}$ wechselt am QKEP das Vorzeichen, weil die Entropie dort ein Maximum durchläuft. (rechts) Die Theorie eines metamagnetischen QKEPs liefert drei charakteristische Bereiche für $\alpha$ (hier ist $d=2$ ). Aus Geg06b].

kritische Feld $H_{\mathrm{c}}$ in die freie Energie eingeht ${ }^{2}$, wird $\alpha$ mit Gleichung $2.10 \mathrm{zu}$

$$
\alpha=-\frac{1}{V}\left(\frac{\partial S}{\partial p}\right)_{T}=-\frac{1}{V}\left(\frac{\partial S}{\partial H}\right)_{T} \frac{\partial H_{\mathrm{c}}}{\partial p}
$$

und mißt damit die Ableitung der Entropie nach dem Kontrollparameter. Die kritischen Fluktuationen erhöhen auch bei endlichen Temperaturen die Entropie, die deshalb beim kritischen Feld $H=H_{\mathrm{c}}$ ein Maximum annimmt. Der Vorzeichenwechsel der thermischen Ausdehnung kann also qualitativ verstanden werden [GR05. Die genaue Form von $\alpha$ ist konsistent mit den Vorhersagen der Hertz-Millis-Theorie eines zweidimensionalen metamagnetischen QKEPs mit longitudinalen Fluktuationen der Magnetisierung beim kritischen Feld [Mil02]. Man unterscheidet die drei im Inset zu Abbildung 4.6 (rechts) gezeigten Bereiche: Im Bereich I gilt $\alpha \propto T$, während es in Bereich II zu Abweichungen von diesem Fermiflüssigkeitsverhalten kommt. Der Übergang in den quantenkritischen Bereich III wird gerade vom Maximum in $\alpha$ markiert. Im Phasendiagramm beschreiben die Orte der Maxima von $\alpha$ den charakteristischen "Trichter" über dem QKP. Die Theorie macht weiterhin Vorhersagen bezüglich des Skalenverhaltens: Danach skaliert z. B. wie in Abbildung 4.6 (rechts) gezeigt, die Temperatur, bei der $\alpha$ sein Maximum annimmt, wie $h^{2 / 3}$. In einer geeigneten Skalenauftragung wird dementsprechend ein Kollaps der bei verschiedenen Magnetfeldern gemessenen Daten beobachtet Geg06b]. Bemerkenswerterweise ist der kritische Beitrag so stark, daß er die thermische Ausdehnung fast vollständig dominiert, so daß für die Analyse keinerlei Hintergrund angenommen bzw. abgezogen werden mußte.

\footnotetext{
${ }^{2}$ Das heißt anschaulich, daß $H$ und $p$ als Kontrollparameter der Quantenkritikalität äquivalent sind.
} 


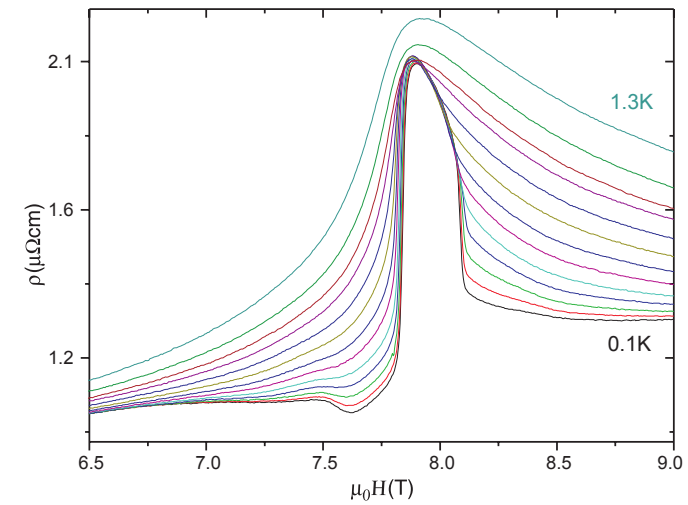

Abbildung 4.7: Nahe des QKEPs beobachtet man in sehr reinen Proben einen Bereich mit stark erhöhtem, temperaturunabhängigen Restwiderstand. Aus Gri04.

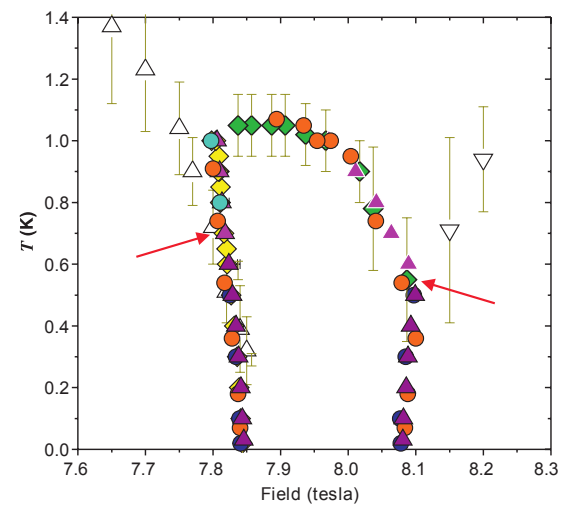

Abbildung 4.8: Anomalien in verschiedenen Meßgrößen deuten übereinstimmend auf die Existenz einer wohldefinierten Phase hin. Unterhalb der roten Pfeile sind die Übergänge erster Ordnung. Aus Gri04].

\subsection{Nematische Phase}

Die Proben, an denen die ersten Untersuchungen $\mathrm{zu} \mathrm{Sr}_{3} \mathrm{Ru}_{2} \mathrm{O}_{7}$ durchgeführt wurden, besaßen mit einem Restwiderstand von $\varrho_{0}=3 \mu \Omega \mathrm{cm}$ bereits eine recht hohe Qualität [Per01, Gri03, Gri01]. Erst mit einer weiteren Optimierung der Probenqualität auf $\varrho_{0}=0,4 \mu \Omega \mathrm{cm}$ zeigte sich ein grundsätzlich neues Verhalten Gri04. Während abseits des QKEPs die Probeneigenschaften im Wesentlichen unabhängig von der Probenreinheit waren, wurde in den sehr sauberen Proben für $B \| c$ in der Nähe des metamagnetischen Übergangs bei 7,8 T ein Bereich mit stark erhöhtem und temperaturunabhängigen Restwiderstand beobachtet, vgl. Abbildung 4.7. Führt man die Daten aus verschiedenen Meßgrößen zusammen, so zeigt sich für $T \leq 1 \mathrm{~K}$ eine wohldefinierte Phase, s. Abbildung 4.8. Sie ist bei tiefen Temperaturen durch zwei metamagnetische Übergänge erster Ordnung begrenzt, welche sich z. B. durch einen nichtverschwindenden Imaginärteil der magnetischen Suszeptibilität äußern. Zu höheren Temperaturen hin sind sie durch eine Linie verbunden, die wahrscheinlich einem Übergang zweiter Ordnung entspricht.

In weiteren Experimenten [Bor07] wurde das Magnetfeld nun um verschiedene Winkel aus der $c$-Richtung verkippt, so daß es eine Komponente in der $a b$-Ebene besitzt. In Anlehnung an die englischsprachige Literatur soll diese im folgenden kurz mit „In-

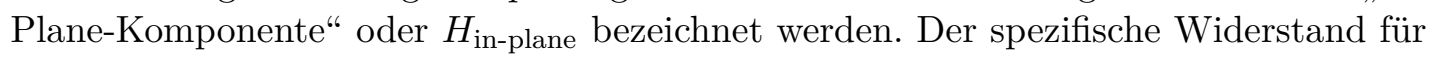
eine Temperatur von $100 \mathrm{mK}$ und einen Kippwinkel von $13^{\circ}$ ist in Abbildung 4.9 für zwei unterschiedliche Konfigurationen gezeigt. Dabei wird $\varrho_{\|}$mit dem Stromfluß parallel zur In-Plane-Komponente des Magnetfelds gemessen, und für $\varrho_{\perp}$ gilt entsprechend $I \perp H_{\text {in-plane. }}$ Während $\varrho_{\|}$(schwarze Kurve) nach wie vor den aus Abbildung 4.7 bekannten erhöhten Restwiderstand aufweist, ist diese Anomalie in $\varrho_{\perp}$ (rote Kurve) unterdrückt. 

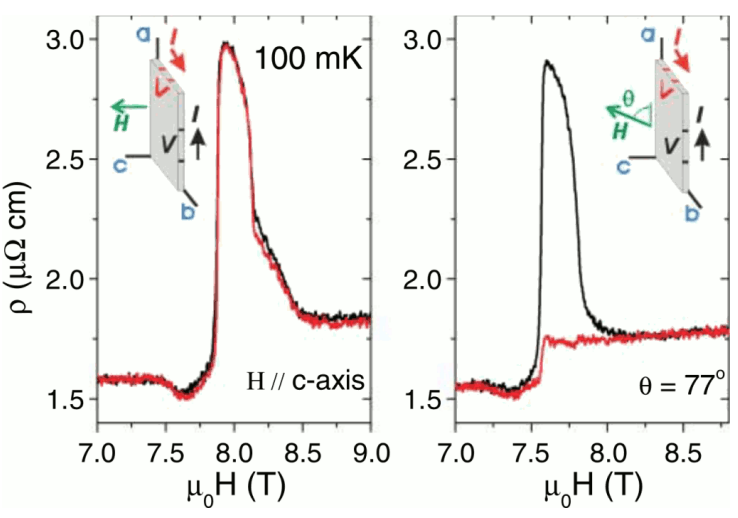

Abbildung 4.9: Ein leichtes Verkippen des Magnetfelds in die $a b$-Ebene erzeugt eine Anisotropie im Transportverhalten. Aus Bor07.

Nach diesem experimentellen Befund kann von einer „leichten“ bzw. „schweren Achse" für den Transport gesprochen werden, und zwar existiert eine

- leichte Achse für $I \perp H_{\text {in-plane }}$ und eine

- schwere Achse für $I \| H_{\text {in-plane. }}$

Je nach der Richtung, in die das Feld verkippt wird, kann die leichte Achse parallel zur $a$ - oder $b$-Richtung des Kristalls gelegt werden, beliebige Richtungen (z. B. $45^{\circ}$ ) sind allerdings nicht möglich. Für größere Kippwinkel verschwindet die Widerstandsanomalie schnell. Neutronenstreuexperimente zeigen oberhalb einer Aufösungsgrenze von $4 \cdot 10^{-5} \AA$ keinen Unterschied der Gitterparameter $a$ und $b$. Offenbar bleibt also die zugrundeliegende Symmetrie des Gitters erhalten, während der elektronische Transport eine Anisotropie aufweist. BORZI ET AL. Bor07] sprachen deshalb von einer elektronischen nematischen Phase. ${ }^{3}$ Als phänomenologischen Ordnungsparameter führten sie die relative Differenz des spezifischen Widerstands

$$
\left(\varrho_{\|}-\varrho_{\perp}\right) /\left(\varrho_{\|}+\varrho_{\perp}\right)
$$

ein. Dieser Ordnungsparameter wird für $T \rightarrow 0$ maximal und geht für $T \rightarrow T_{\mathrm{c}}$ kontinuierlich gegen Null.

\subsubsection{Domänenmodell}

Da beim Eintritt in die nematische Phase keine wesentliche Änderung des Hallkoeffizienten stattfindet Gri04, fällt eine Änderung der Ladungsträgerkonzentration (z. B. durch Lokalisierung von Ladungsträgern) als Erklärung für den erhöhten Restwiderstand aus. Dieser muß vielmehr in einem itineranten Szenario beschrieben werden, wobei er durch elastische Streuung hervorgerufen wird.

GRIGERA et al. Gri04 postulierten eine spontane symmetriebrechende Verzerrung der Fermifläche. Diese Verzerrung hätte dabei zwei um $90^{\circ}$ gegeneinander gedrehte Möglichkeiten, sich relativ zum Gitter auszurichten, so daß sich Domänen bilden

\footnotetext{
${ }^{3}$ Vgl. zum Begriff der Nematizität den Abschnitt 4.6 weiter unten.
} 
könnten. Der erhöhte Wert von $\varrho_{0}$ ließe sich dann durch Streuung an den Grenzen zwischen diesen Domänen erklären. Gäbe es weiterhin einen Mechanismus, durch den das In-Plane-Feld die Domänen ausrichten könnte, so ließe sich außerdem das Auftreten einer leichten Richtung verstehen, in der die anomale Streuung unterdrückt wird. Für größere Kippwinkel, bei denen die Anisotropie verschwindet, gibt es zwei mögliche Mechanismen: Entweder verschwindet die nematische Ordnung ganz, oder die Domänen sind vollständig ausgerichtet, so daß nur noch ein Typ von Domäne existiert, also auch keine Domänenwände und damit keine Streuung mehr vorhanden sind.

Ein Szenario mit Domänenbildung wird unterstützt durch Messungen des de-Haasvan-Alphen(dHvA)-Effektes. Mercure et al. Mer09; Mer10 finden innerhalb der nematischen Phase zwei dHvA-Frequenzen, die mit den $\beta$ - und $\alpha_{1}$-Orbits identifiziert werden können. Da die Temperaturabhängigkeit ihrer Amplituden der Lifshitz-KosevitchTheorie gehorcht, ist es wahrscheinlich, daß die dHvA-Oszillationen von Landauschen Quasiteilchen stammen. Innerhalb der Phase findet man also Fermiflüssigkeitsverhalten. Das Verhältnis der Amplituden unterschiedlicher Orbits außerhalb der Phase ist durch den Dingle-Faktor $R_{\mathrm{D}}=\exp \left(-\pi \hbar k_{\mathrm{F}} / e l B\right)$ gegeben, welcher die magnetfeldabhängige Dämpfung der Oszillationen durch Streuung an homogen verteilten Störstellen beschreibt. Elektronen auf größeren Orbits (mit größerem Fermiwellenvektor $k_{\mathrm{F}}$ ) müssen pro Umlauf eine größere Strecke zurücklegen, so daß die Zyklotronresonanzbedingung mit größerer Wahrscheinlichkeit durch Streuung verletzt wird. Die aus der Dingle-Analyse extrahierte mittlere freie Weglänge $l=135 \mathrm{~nm}$ ist konsistent mit dem aus dem Restwiderstand erhaltenen Wert. Innerhalb der Phase folgt die Dämpfung aber nicht mehr dem erwarteten Verlauf: vielmehr werden Oszillationen mit großem Fermiflächenquerschnitt, also einer großen Bahnfläche im Realraum, überproportional, d. h. noch stärker, als es nach dem Dinglefaktor zu erwarten wäre, gedämpft. Dies ließe sich so erklären, daß der dHvA-Effekt nur für Kreisbahnen, die vollständig innerhalb einer Domäne liegen, beobachtet werden kann. Für größere Orbits würde die Oszillation durch Streuung an den Domänenwänden zerstört. Unter dieser Annahme erhält man einen mittleren Domänendurchmesser von der Größenordnung $500 \mathrm{~nm}$.

\subsection{Spezifische Wärme und Entropie}

Rost et al. Ros09 haben Experimente zum magnetokalorischen Effekt in $\mathrm{Sr}_{3} \mathrm{Ru}_{2} \mathrm{O}_{7}$ vorgenommen und das Verhalten von Entropie und spezifischer Wärme im Phasendiagramm untersucht. Während die seitlichen Phasengrenzlinien schon in früheren Arbeiten als Phasenübergänge erster Ordnung klassifiziert wurden, konnte hier erstmalig auch für das „Dach" eine deutliche thermodynamische Signatur gefunden werden. Abbildung 4.10 (links) zeigt einen deutlichen Sprung der spezifischen Wärme, was mit einem Phasenübergang zweiter Ordnung konsistent ist. Auffällig ist allerdings die Übergangstemperatur von $1,2 \mathrm{~K}$, die signifikant höher liegt als die aus anderen Größen bestimmte, vgl. Abbildung 4.8 . 

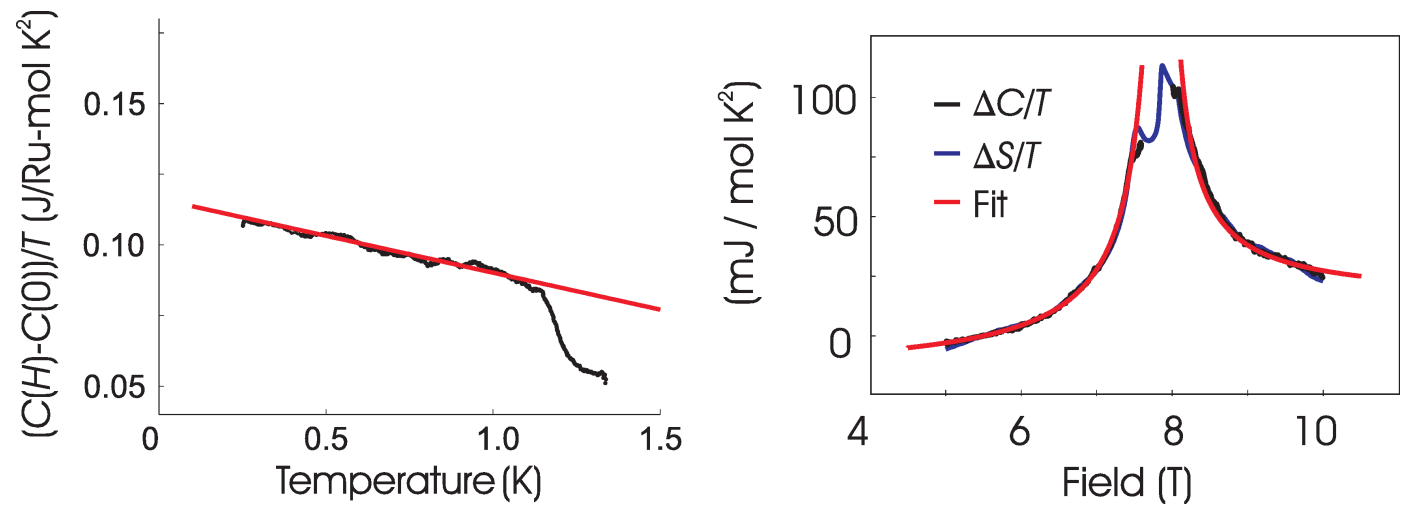

Abbildung 4.10: Spezifische Wärme von $\mathrm{Sr}_{3} \mathrm{Ru}_{2} \mathrm{O}_{7}$ aus [Ros09]. Links: in der Nähe des Phasendachs zeigt $c(T)$ für $B=7,9 \mathrm{~T}$ einen Sprung bei ca. $1,2 \mathrm{~K}$. Rechts: Bei $T=0,25 \mathrm{~K}$ lassen sich Entropie und spezifische Wärme durch $\Delta c(H)=\Delta S(H) \propto T \cdot\left(H-H_{\mathrm{c}}\right)^{-1}$ beschreiben.

Im Fermiflüssigkeitsbereich bei tiefen Temperaturen gilt

$$
c=\gamma T=T \frac{\partial S}{\partial T} \Rightarrow S=\int \gamma \mathrm{d} T=\gamma T+\text { const. }=c+\text { const. },
$$

so daß Entropie und spezifische Wärme bis auf eine Konstante identisch sind. Wie Abbildung 4.10 (rechts) zeigt, steigen beide Größen auf beiden Seiten des kritischen Felds an und durchlaufen im kritischen Bereich ein Maximum. Ein Maximum der Entropie beim kritischen Wert des Kontrollparameters ist eine zentrale Eigenschaft eines Quantenphasenübergangs, die auch zum in Abschnitt 4.3 besprochenen Vorzeichenwechsel in der thermischen Ausdehnung führt. Der Verlauf des kritischen Anteils der spezifischen Wärme läßt sich für $H<H_{\mathrm{c}}$ durch eine Divergenz der Form $\Delta c / T \propto\left[\left(H-H_{\mathrm{c}}\right) / H_{\mathrm{c}}\right]^{-1 \pm 0,1}$ anpassen. In Ros10 wird gezeigt, daß sich eine Divergenz der Form $\left(H-H_{\mathrm{c}}\right)^{-1}$ aus einer entsprechend konstruierten Bandstruktur mit van-Hove-Singularitäten ableiten läßt.

Der beobachtete Exponent scheint im Widerspruch zur Hertz-Millis-Theorie, welche die thermische Ausdehnung korrekt beschreibt, für die spezifische Wärme allerdings $c \propto h^{-1 / 3}$ vorhersagt, vgl. Abschnitt 2.3. Dabei ist allerdings zu berücksichtigen, daß der kritische Anteil $\Delta c / T$ auf einem verhältnismäßig großen Hintergrund analysiert wird, der im Nullfeld bereits etwa $110 \mathrm{~mJ} / \mathrm{molRuK}^{2}$ beträgt. Der quantenkritische Beitrag dominiert nur sehr nahe am QKP, gerade dort wird er aber durch die Bildung einer neuen Phase abgeschnitten. In der thermischen Ausdehnung übersteigt dagegen der kritische Beitrag den Hintergrund um Größenordnungen.

Die Größe $S / T$ zeigt für $H<H_{\mathrm{c}}$ ebenso eine Divergenz, welche allerdings im Bereich der nematischen Phase abgeschnitten wird. In einem einfachen qualitativen Bild vermeidet das System damit eine unphysikalische Singularität der Entropie beim QKP durch das Einnehmen eines neuen geordneten Zustands, in welchem die quantenkritischen Fluktuationen unterdrückt sind. Dies ist ein weiterer Hinweis auf eine ursächliche Verbindung zwischen Quantenkritikalität und der nematischen Phase Ros09. 
Im Phasendiagramm in Abbildung 4.8 fällt auf, daß die Phasengrenzlinien bei tiefen Temperaturen nach außen geneigt sind. Für einen Phasenübergang erster Ordnung ist die Steigung der Koexistenzkurve $B_{\mathrm{c}}(T)$ über die Clausius-Clapeyron-Gleichung

$$
\frac{\mathrm{d} B_{\mathrm{c}}}{\mathrm{d} T}=\frac{S_{1}-S_{2}}{M_{2}-M_{1}}
$$

mit den Unterschieden von Entropie und Magnetisierung verknüpft, wobei der Index 1 (2) jeweils die Phase bei tieferen (höheren) Feldern bezeichnen soll. Da die Magnetisierung bei den metamagnetischen Übergängen einen Sprung mit positivem Vorzeichen macht, ist $m_{2}-m_{1}>0$. Die Steigung der Phasengrenzlinie ist beim ersten Übergang negativ, so daß $S_{2}>S_{1}$, Entsprechendes gilt für den zweiten Übergang. Damit folgt aus (4.5), daß die nematische Phase eine höhere Entropie besitzt als ihre Umgebung. Dies konnte auch experimentell verifiziert werden: Rost et al. Ros09 finden in Messungen des magnetokalorischen Effekts (Gleichung (2.16) ) einen Sprung der Entropie, der quantitativ mit dem aus (4.5) berechneten übereinstimmt. Dies ist ein starker Hinweis darauf, daß es sich beim metamagnetischen Übergang tatsächlich um einen Phasenübergang erster Ordnung zwischen thermodynamischen Gleichgewichtsphasen handelt.

Die erhöhte Entropie ist ein äußerst überraschendes Ergebnis, da bei Phasenübergängen zweiter Ordnung die Tieftemperaturphase i. A. durch einen spontanen Symmetriebruch geordnet ist und damit eine niedrigere Entropie besitzt. Eine mögliche Interpretation ist eine Erhöhung der Unordnung durch die oben besprochene Existenz von Domänen unterschiedlicher Orientierung. Gegen eine solche Annahme spricht allerdings die Größe der Domänen, die bei einem mittleren Durchmesser von $500 \mathrm{~nm}$ in der Ebene etwa $10^{4}$ Einheitszellen enthalten sollten. Ihr Entropiebeitrag wäre also gegenüber dem der Ru-Atome einer einzigen Einheitszelle zu vernachlässigen. Möglicherweise spielen deshalb auch Fluktuationseffekte zwischen Domänen unterschiedlicher Orientierung eine Rolle Rag09].

\subsection{Nematizität}

Nematizität bezeichnet einen Zustand, in dem die Rotationssymmetrie eines Systems gebrochen wird, während die Translationssymmetrie erhalten bleibt. In der nematischen Phase eines Flüssigkristalls beispielsweise sind die stäbchenförmigen Moleküle bezüglich ihrer Orientierung geordnet („Kristall“), bzgl. der Position jedoch ungeordnet („Flüssigkeit“) Set91. Ursächlich für die Physik des Gesamtsystems ist hier die längliche Form der einzelnen Teilchen. In den letzten 10 Jahren gaben nun mehrere Experimente Hinweise auf die Existenz von elektronischen nematischen Zuständen in unterschiedlichen Systemen [Fra10], darunter auch $\mathrm{Sr}_{3} \mathrm{Ru}_{2} \mathrm{O}_{7}$. Wie können aber punktförmige Elektronen einen nematischen Zustand einnehmen?

Ein Beispiel sind zweidimensionale Elektronengase (2DEG), wie sie z. B. in GaAsGaAlAs-Heterostrukturen realisiert werden können. Diese Systeme sind bekannt für 
das Auftreten des ganzzahligen und gebrochenzahligen Quantenhalleffekts KDP80; TSG82 bei tiefen Temperaturen und hohen Magnetfeldern. Im Längswiderstand wurde zusätzlich eine starke Anisotropie $\varrho_{x x} / \varrho_{y y} \approx 100$ beobachtet, je nachdem, in welcher Richtung dieser gemessen wird [Lil99]. Da die Anisotropie nur bei halber Füllung bestimmter Landauniveaus auftritt, anderweitig aber nicht zu finden ist, kann eine intrinsische, möglicherweise durch die Herstellung bedingte Anisotropie der Probe ausgeschlossen werden. Die Ursache liegt vielmehr in der Bildung von unidirektionalen Ladungsdichtewellen, die sich in einem Streifenmuster mit einer Längenskala von ca. $100 \mathrm{~nm}$ anordnen KFS96.

Der Effekt tritt für ein senkrecht angelegtes Magnetfeld nur für sehr tiefe Temperaturen $T \leq 100 \mathrm{mK}$ auf, während die Theorie die Bildung von Ladungsdichtewellen bereits für Temperaturen von einigen Kelvin vorhersagt. Ein Verkippen des Magnetfelds in die Ebene läßt die Anisotropie aber auch bei höheren Temperaturen hervortreten. Außerdem wird nun auch für Füllfaktoren, für die der Widerstand vorher isotrop war, die Symmetrie gebrochen. Dies spricht dafür, daß lokale Streifenordnung in Form von Domänen bereits bei höheren Temperaturen auftritt, aber von thermischen Fluktuationen zerstört wird, so daß sich keine langreichweitige Ordnung ausbilden kann.

Die Beobachtungen in 2DEGn (anisotroper Transport, der durch ein Magnetfeld in der Ebene beeinflußt werden kann) sind bemerkenswert ähnlich zu denen in $\mathrm{Sr}_{3} \mathrm{Ru}_{2} \mathrm{O}_{7}$, allerdings muß auch auf grundlegende Unterschiede hingewiesen werden: Die Elektronendichte in den Quanten-Hall-Systemen beträgt $\varrho_{2 \mathrm{D}} \approx 3 \cdot 10^{11} \mathrm{~cm}^{-2}$, denn nur eine so starke Verdünnung ermöglicht Experimente in den niedrigsten Landauniveaus. Im dreidimensionalen Fall entspricht dies immer noch $\varrho_{3 \mathrm{D}}=\varrho_{2 \mathrm{D}}^{3 / 2} \approx 2 \cdot 10^{17} \mathrm{~cm}^{-3}$. Dieser Wert liegt 5-6 Größenordnungen unter der Elektronendichte eines Metalls wie $\mathrm{Sr}_{3} \mathrm{Ru}_{2} \mathrm{O}_{7}$.

Etwas weniger deutliche, aber hochinteressante Hinweise auf elektronische nematische Ordnung gibt es in den Hochtemperatursupraleitern $\mathrm{YBaCuO}$ und $\mathrm{LaSrCuO}$. Dort beobachtet man in den $\mathrm{CuO}$-Ebenen einen anisotropen spezifischen Widerstand mit einem Verhältnis $\varrho_{a} / \varrho_{b} \approx 2$, der zu tiefen Temperaturen hin steigt [And02]. Zwar ist die Kristallstruktur jeweils orthorhombisch, doch steigende Dotierung verringert die Orthorhombizität, während sie die Anisotropie verstärkt, so daß die Symmetrie des Gitters als Ursache ausgeschlossen werden kann. Messungen des Nernst-Effekts zeigen, daß das Einsetzen der Anisotropie mit dem Eintritt in die Pseudogap-Phase zusammenfällt, die damit als symmetriegebrochener elektronischer Zustand identifiziert wird Dao10. Ähnliche Beobachtungen gibt es auch in den z.Zt. intensiv untersuchten Eisenarsenidsupraleitern. Anders als die Kuprate, die im undotierten Zustand Mott-Isolatoren sind, zeichnen diese Systeme sich durch einen metallischen Grundzustand aus, dessen Antiferromagnetismus durch Dotierung unterdrückt wird und in einen supraleitenden Zustand mit Sprungtemperaturen von bis zu $55 \mathrm{~K}$ mündet PG10. Der antiferromagnetischen Ordnung geht ein struktureller Phasenübergang voraus, der möglicherweise von einem elektronischen Ordnungsübergang getrieben wird. Widerstandsanisotropien in diesem Bereich geben Hinweise auf die Existenz nematischer Ordnung [Chu10]. 


\subsubsection{Theorien für $\mathrm{Sr}_{3} \mathrm{Ru}_{2} \mathrm{O}_{7}$}

Wie läßt sich nun die Entstehung der nematische Phase in $\mathrm{Sr}_{3} \mathrm{Ru}_{2} \mathrm{O}_{7}$ erklären? GRIGERA et al. Gri04 postulierten eine spontane symmetriebrechende Fermiflächenverzerrung. Ein solcher Effekt wurde erstmals von Pomeranchuk [Pom58] im Rahmen der Fermiflüssigkeitstheorie diskutiert und wird seitdem als Pomeranchuk-Instabilität bezeichnet. Dabei wird die Fermifläche unter bestimmten Voraussetzungen instabil und erniedrigt ihre Symmetrie. Im Experiment wurde eine Pomeranchuk-Instabilität seitdem allerdings noch nicht beobachtet.

Für eine Theorie der nematischen Phase sind zwei Aspekte wesentlich:

1. Es liegt, wie oben besprochen, quantenkritisches Verhalten vor. In der Nähe eines quantenkritischen Punktes entstehen oftmals neuartige Phasen wie z. B. die Schwere-Fermionen-Supraleitung [GSS08. Auch für die Hochtemperatursupraleiter wird ein „verborgener" quantenkritischer Punkt diskutiert [Bro08]. Quantenkritische Fluktuationen spielen also auch im Fall von $\mathrm{Sr}_{3} \mathrm{Ru}_{2} \mathrm{O}_{7}$ möglicherweise eine wichtige Rolle.

2. Es gibt eine komplizierte Bandstruktur mit orbitalem Charakter und Metamagnetismus, welcher durch van-Hove-Singularitäten in der elektronischen $\mathrm{Zu}$ standsdichte hervorgerufen wird. Es könnte sich bei der Nematizität also auch um einen Bandstruktureffekt handeln.

Es ist nicht klar, ob diese zwei Aspekte unabhängig voneinander sind, in den letzten Jahren haben sich theoretische Anstrengungen allerdings stärker auf den zweiten Punkt konzentriert.

BERRIDGe et al. Ber09, Ber10 betrachten dazu ein Stoner-Modell mit einer Spitze in der Zustandsdichte und führen eine Ginzburg-Landau-Entwicklung in der Nähe der kritischen Endpunkte durch. Sie finden einen Zustand räumlich modulierter Magnetisierung, der ein magnetisches Analogon zum FFLO-Zustand in Supraleitern darstellt. Ein solcher inhomogener Zustand würde sich durch richtungsabhängige Streuung auszeichnen und damit die Widerstandsanisotropie erklären, allerdings kann das Modell keine Erklärung für die Ausrichtung dieser Anisotropie durch das Magnetfeld liefern.

RAghu et al. Rag09 nähern sich der nematischen Phase im Rahmen eines TightBinding-Modells unter Einbeziehung der Spin-Bahn-Kopplung und können damit eine realistischere Modellierung der Bandstruktur von $\mathrm{Sr}_{3} \mathrm{Ru}_{2} \mathrm{O}_{7}$ erreichen, die aus den lokalen Wellenfunktionen $d_{x y}, d_{x z}$ und $d_{y z}$ der Ru-Atome aufbaut. Ein nematischer Ordnungsparameter ergibt sich dabei aus dem Besetzungsunterschied zwischen den quasi-eindimensionalen $d_{x z^{-}}$und den $d_{y z}$-Bändern. Auch dieses Modell kann die Beobachtungen aus den Widerstandsmessungen nicht abschließend erklären. 


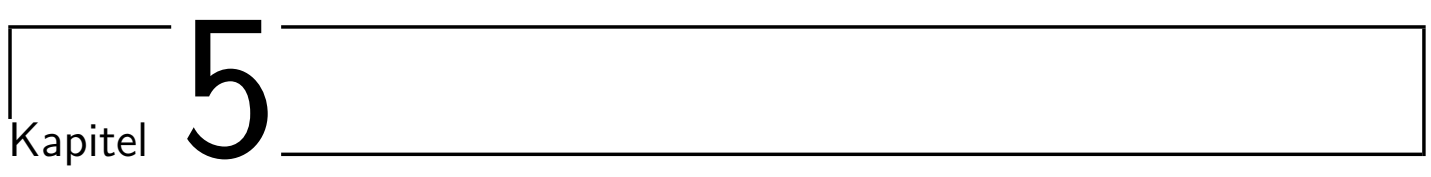

\section{Messungen und Ergebnisse}

Im letzten Abschnitt wurde die „nematische Phase“ in $\mathrm{Sr}_{3} \mathrm{Ru}_{2} \mathrm{O}_{7}$ beschrieben und die Frage nach dem korrekten Ordnungsparameter dieser Phase aufgeworfen. Frühere Arbeiten haben gezeigt, daß es eine starke Kopplung zwischen elastischen und magnetischen Eigenschaften gibt. Dies motiviert eine Untersuchung der nematischen Phase mit der Methode der kapazitiven Dilatometrie. Es soll dabei analog vorgegangen werden wie in den in Abbildung 4.9 gezeigten Widerstandsexperimenten: zuerst wird ein Magnetfeld von ca. $8 \mathrm{~T}$ parallel zur c-Richtung angelegt, um das System auf den quantenkritischen Punkt einzustellen. Danach wird der Einfluß eines leicht in die $a b$-Ebene verkippten Magnetfelds untersucht. Für die spätere Diskussion werden ergänzend auch einige Messungen der spezifischen Wärme einbezogen.

Die in diesem Abschnitt vorgestellten Daten wurden an einer hochreinen $\mathrm{Sr}_{3} \mathrm{Ru}_{2} \mathrm{O}_{7}$ Probe $^{1}$ gemessen, welche von R. PERRY Per04 in der Arbeitsgruppe von Y. MAENO an der Universität von Kyoto im Zonenschmelzverfahren hergestellt wurde. Das Probenstück stammt vom selben Einkristall, der bereits in Gri04 Geg06b Geg06a untersucht wurde, womit eine gute Vergleichbarkeit der Ergebnisse gewährleistet ist. Mittels Funkenerosion wurden senkrecht zu den zu messenden Richtungen je zwei planparallele Flächen hergestellt. Die Abmessungen der Probe betragen danach 1,2 $\mathrm{mm}$ in der $c$-Richtung und $1,5 \mathrm{~mm}$ in der $a$-Richtung.

\subsection{Dilatometrie an $\mathrm{Sr}_{3} \mathrm{Ru}_{2} \mathrm{O}_{7}$}

Abbildung 5.1 zeigt schematisch die unterschiedlichen Konfigurationen, die für diese Arbeit untersucht wurden. Die gemessene Längenänderung $\Delta L$ liegt dabei (bis auf einige Messungen am Anfang dieses Kapitels) immer in der ab-Ebene und ist parallel zu einer [100]-Richtung des pseudotetragonalen Gitters (vgl. Abschnitt 4.1). Das Magnetfeld $\vec{B}$ ist parallel zur $c$-Achse und kann nun um einen Winkel $\Phi=5^{\circ}, 10^{\circ}, 15^{\circ}$ verkippt werden, so daß eine Komponente von $B$ in der $a b$-Ebene entsteht. Wie schon

\footnotetext{
${ }^{1}$ Die genaue Bezeichnung der Probe lautet Nr. C660a.
} 


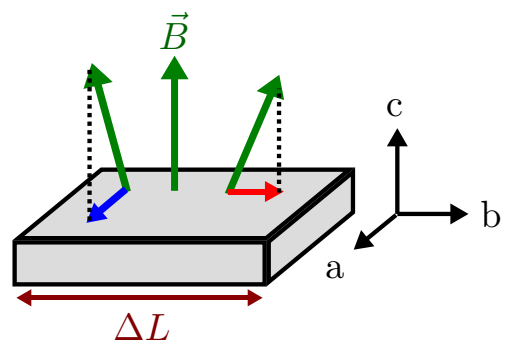

Abbildung 5.1: Die Längenänderung $\Delta L$ wurde in zwei unterschiedlichen Konfigurationen bezüglich der

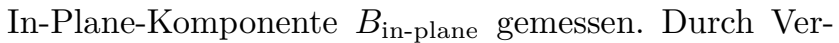
kippen von $\vec{B}$ um unterschiedliche Achsen ergibt sich $\Delta L \| B_{\text {in-plane }}\left(\right.$ rot) und $\Delta L \perp B_{\text {in-plane }}$ (blau).

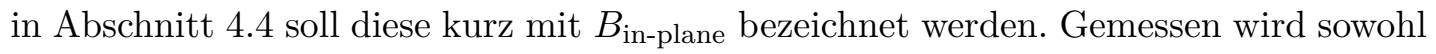
die Längenänderung parallel ( $\left.\Delta L \| B_{\text {in-plane }}\right)$ als auch senkrecht $\left(\Delta L \perp B_{\text {in-plane }}\right) \mathrm{zu}$ dieser Komponente. Je nach Konfiguration werden im folgenden die Größen $\Delta L, \alpha$ und $\lambda$ mit einem entsprechenden Index versehen, also $\Delta L_{\|}, \alpha_{\perp}$ usw.

Da die Richtung des Magnetfelds durch die Lage des fest installierten Supraleitungsmagneten vorgegeben ist, wird die Verkippung im Experiment durch eine Rotation des Dilatometers um seine Befestigungsachse (vgl. Abbildung 3.2 oder durch eine Rotation der Probe um die Meßrichtung (in einem für den jeweiligen Winkel gefertigten Probenhalter, Bauteil 6 in Abbildung 3.1) realisiert. Die zwei Konfigurationen unterscheiden sich also durch die Wahl der Drehachse, während jeweils dieselbe physikalische Achse der Probe gemessen wird. Aufgrund der begrenzten Größe der Anordnung ist die Ausrichtung mit einem Fehler behaftet, der schätzungsweise $\Delta \Phi \leq 2^{\circ}$ beträgt. Da eine Änderung des Winkels oder ein Wechsel der Konfiguration mindestens einen Ausbau des Mischungseinsatzes und meist sogar einen Ausbau der Probe aus dem Dilatometer erfordert, kann sich $\Delta \Phi$ zwischen zwei Messungen entsprechend ändern.

\subsubsection{Unverkippte Konfiguration}

Um die Vergleichbarkeit mit existierenden Meßdaten sicherzustellen, wurde zunächst die Magnetostriktion für $B \| \mathrm{c}$ untersucht, die bereits aus Geg06a Gri04 bekannt ist. Abbildung 5.2 (links) zeigt hierfür die Längenänderung als Funktion des Magnetfelds und den hieraus berechneten Magnetostriktionskoeffizienten $\lambda$ für verschiedene Temperaturen. Bei tiefen Temperaturen zeigen sich in $\lambda$ drei Maxima bei 7,53 T, 7,85 T und $8,09 \mathrm{~T}$, von denen das mittlere bei $B=7,85 \mathrm{~T}$ das am stärksten ausgeprägte ist. Es handelt sich bei den Maxima um die bekannten metamagnetischen Übergänge, wobei der zweite und dritte erster Ordnung sind, während der erste Übergang als Crossover eingeordnet wird Gri04. Die nematische Phase wird, wie in Abschnitt 4.4 beschrieben, vom zweiten und dritten Übergang eingeschlossen. Mit steigender Temperatur werden die Maxima niedriger und breiter und verschieben sich außerdem zu kleineren Feldern. Die Magnetostriktion ist im gesamten Feldbereich positiv; insbesondere wächst die Länge sowohl beim Eintritt als auch beim Austritt aus der nematischen Phase. Insgesamt ist eine sehr gute Übereinstimmung mit den Daten aus vorherigen Arbeiten festzustellen.

Im folgenden werden nun ausschließlich Messungen in der Ebene behandelt. 

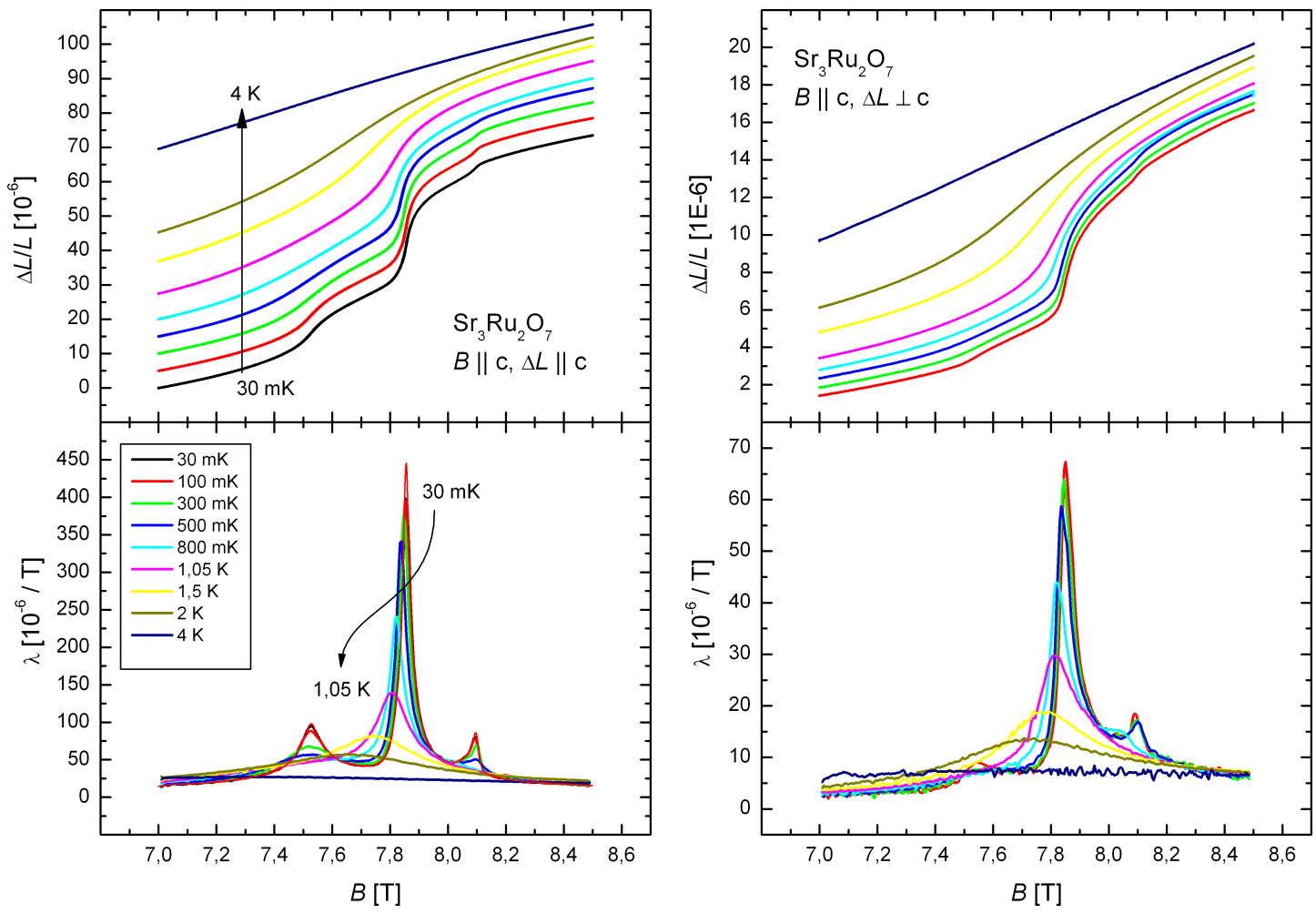

Abbildung 5.2: Magnetostriktion von $\mathrm{Sr}_{3} \mathrm{Ru}_{2} \mathrm{O}_{7}$ parallel (links) und senkrecht (rechts) zur $c$-Achse. In beiden Richtungen werden die drei metamagnetischen Übergänge beobachtet. Die Kurven für die Längenänderung sind in vertikaler Richtung willkürlich verschoben. 
Magnetostriktion Die Magnetostriktion in der Ebene (Abbildung 5.2 rechts) zeigt ein qualitativ ähnliches Verhalten. Auch hier ist $\lambda$ durchweg positiv, allerdings um einen Faktor $6-7$ kleiner. Der metamagnetische Crossover bei 7,53 T ist deutlich schwächer ausgeprägt und läßt sich nur bei den niedrigsten Temperaturen auflösen.

Aus den Positionen der Maxima von $\lambda$ läßt sich nun das Phasendiagramm in Abbildung 5.6 konstruieren. Die Daten zeigen eine gute Übereinstimmung mit den aus den Maxima der magnetischen Wechselfeldsuszeptibilität gewonnenen Punkten [Gri04], wobei insbesondere beim metamagnetische Hauptübergang eine systematische Verschiebung um ca. $10 \mathrm{mT}$ zu höheren Feldern auffällt. Für diese Verschiebung gibt es zwei mögliche Erklärungsansätze: Einerseits tritt in supraleitenden Magneten häufig ein geringes remanentes Feld auf, da es sich bei der für die Windungen verwendeten Legierung $\mathrm{Nb}_{3} \mathrm{Sn}$ um einen Supraleiter zweiter Art handelt. Da die Verschiebung jedoch bei mehreren Messungen mit unterschiedlicher Vorgeschichte des Magneten in gleicher Weise beobachtet wird, ist ein anderer Effekt wahrscheinlicher: Nach Abschnitt 3.2.4 wirkt im Dilatometer prinzipbedingt eine Kraft von etwa $3 \mathrm{~N}$ auf die Probe, welche bei einer Auflagefläche von ca. 1,85 mm² einem uniaxialen Druck von ca. 16 bar entspricht. Die hydrostatische Druckabhängigkeit des metamagnetischen Feldes von $\mathrm{Sr}_{3} \mathrm{Ru}_{2} \mathrm{O}_{7}$ wurde abgeschätzt zu d $B_{\mathrm{mm}} / \mathrm{d} p=5,6 \mathrm{~T} / \mathrm{GPa}$ Chi02: Geg06a, was eine Verschiebung von 9,1 mT bedeutet. Die Verschiebung des metamagnetischen Übergangs wird damit quantitativ erklärt. Außerdem ist der Magnetostriktionskoeffizient über die Maxwellrelation

$$
\lambda V=-\left(\frac{\partial M}{\partial p}\right)_{B}
$$

mit der Druckabhängigkeit der Magnetisierung verknüpft. Das positive Vorzeichen von $\lambda$ impliziert also eine unter Druck geringer werdende Magnetisierung. Diese Beobachtung ist qualitativ konsistent mit der Verschiebung des metamagnetischen Feldes unter uniaxialem Druck.

Thermische Ausdehnung Als nächstes soll die thermische Ausdehnung in der Ebene betrachtet werden. Die entsprechenden Daten in $c$-Richtung sind bereits aus früheren Untersuchungen bekannt, vgl. Abbildung 4.6 auf Seite 43 . In Abbildung 5.3 (links) ist der thermische Ausdehnungskoeffizient $\alpha$, der durch lineare Anpassungen über Intervalle von $80 \mathrm{mK}$ bestimmt wurde, zunächst für Magnetfelder außerhalb der nematischen Phase gezeigt. Das Verhalten ist qualitativ recht ähnlich wie in der $c$-Richtung: es zeigt sich ein Maximum in $\alpha$, welches mit Annäherung an das kritische Feld bei 7,85 T schmaler und höher wird und sich zu tieferen Temperaturen verschiebt. Mit einem maximalen Wert von $3,5 \cdot 10^{-6} \mathrm{~K}^{-1}$ bei $B=7,8 \mathrm{~T}$ ist die thermische Ausdehnung um einen Faktor 3 kleiner als in $c$-Richtung. Für hohe Temperaturen geht $\alpha$ nicht, wie es theoretisch zu erwarten wäre, gegen 0, sondern es bleibt ein konstanter Hintergrund von $0,5 \cdot 10^{-6} \mathrm{~K}^{-1}$ erhalten. Dieser ist von gleicher Größe wie in der $c$-Richtung, macht sich aber aufgrund der kleineren Beträge des kritischen Anteils in der $a b$-Ebene stärker bemerkbar. 


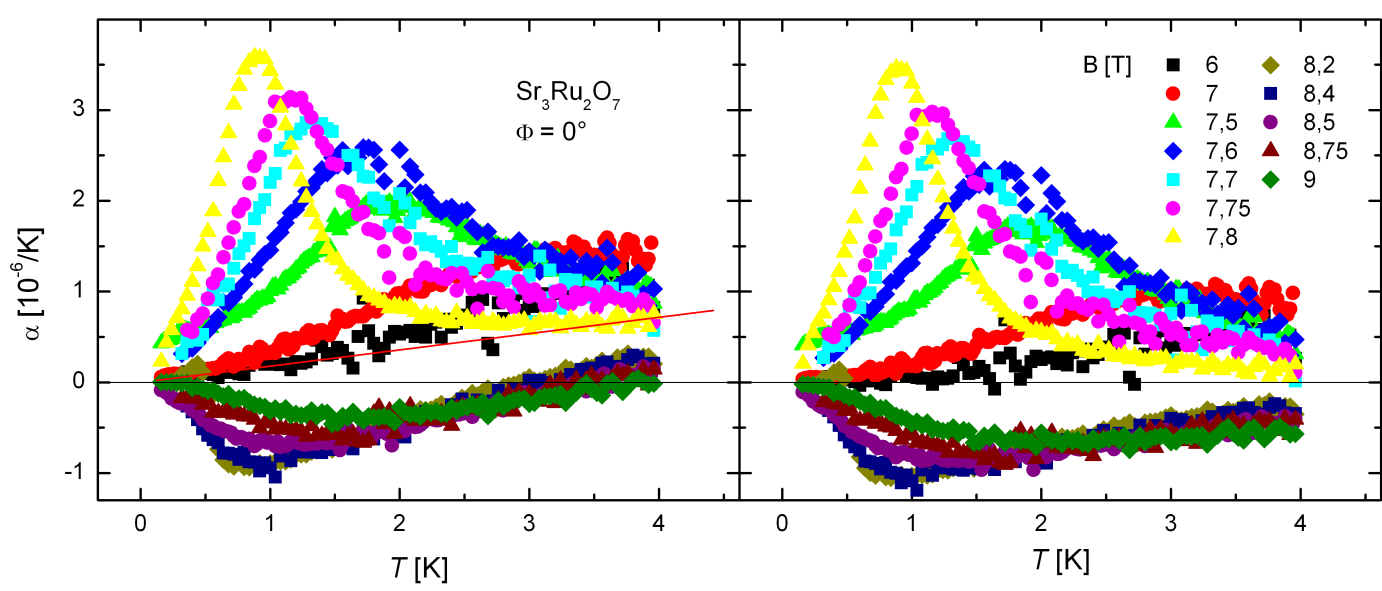

Abbildung 5.3: Links: Thermische Ausdehnung in der $a b$-Ebene für Magnetfelder außerhalb der nematischen Phase. Rechts: Nach Subtraktion eines FF-Hintergrunds (rote Linie im linken Bild) zeigt sich ein quantenkritisches Verhalten, wie es bereits in der c-Richtung beobachtet wurde.

Wie auch in der $c$-Richtung durchläuft $\alpha$ in der Ebene einen Vorzeichenwechsel beim kritischen Feld. Bei höheren Temperaturen wird $\alpha$ in der Ebene im Gegensatz zur $c$ Richtung allerdings auch für $H>H_{\mathrm{c}}$ wieder positiv. Auch hier ist also anscheinend der relative Einfluß eines Hintergrunds stärker als in der $c$-Richtung, wo für die kritische Analyse kein Hintergrundsbeitrag abgezogen werden mußte. In der $a b$-Ebene ist dies aber offensichtlich notwendig.

Für $B=7,8 \mathrm{~T}$ scheint der Hintergrund temperaturunabhängig zu sein. Das Abziehen eines konstanten Hintergrunds in $\alpha$ ist aber nicht mit dem dritten Hauptsatz der Thermodynamik vereinbar, der verlangt, daß $\lim _{T \rightarrow 0}(\alpha)=0$. Es wird deshalb das Verhalten einer Fermiflüssigkeit mit $\alpha(T)=0,14 \cdot 10^{-6} \mathrm{~K}^{-2}$ subtrahiert, wodurch sich die in Abbildung 5.3 (rechts) aufgetragenen Daten ergeben.

Einen Überblick über das Verhalten des kritischen Anteils von $\alpha$ in der $(B, T)$-Ebene gibt die Abbildung 5.4. Orte mit $\alpha=0$ sind in schwarzer Farbe codiert; es zeigt sich, daß der Nulldurchgang von $\alpha$, wie theoretisch erwartet und auch in der $c$-Richtung gemessen Geg06b], temperaturunabhängig ist. Dies spricht dafür, daß der für die Subtraktion angenommene Hintergrund plausibel ist. Auch die als weiße Symbole eingetragene Temperaturen extremaler thermischer Ausdehnung zeigen eine ähnlich Feldabhängigkeit wie in der $c$-Richtung, wobei die Übereinstimmung auf der Niederfeldseite etwas schlechter ist.

Im folgenden soll die Längenänderung als Funktion der Temperatur für Magnetfelder im nematischen Bereich behandelt werden. Eine solche Messung ist in Abbildung 5.5 gezeigt. Das auffälligste Merkmal ist das Auftreten einer deutlichen spontanen Längenänderung bei $T \approx 1 \mathrm{~K}$. Der Sprung hat eine Höhe von $\Delta L / L \approx 1 \cdot 10^{-7}$, was bei einer Probenlänge von $L=1,5 \mathrm{~mm}$ einer Längenänderung von $\Delta L \approx 1,5 \AA$ entspricht. Der Effekt ist also außerordentlich klein. Der im Inset zu Abbildung 5.5 gezeigte thermische 

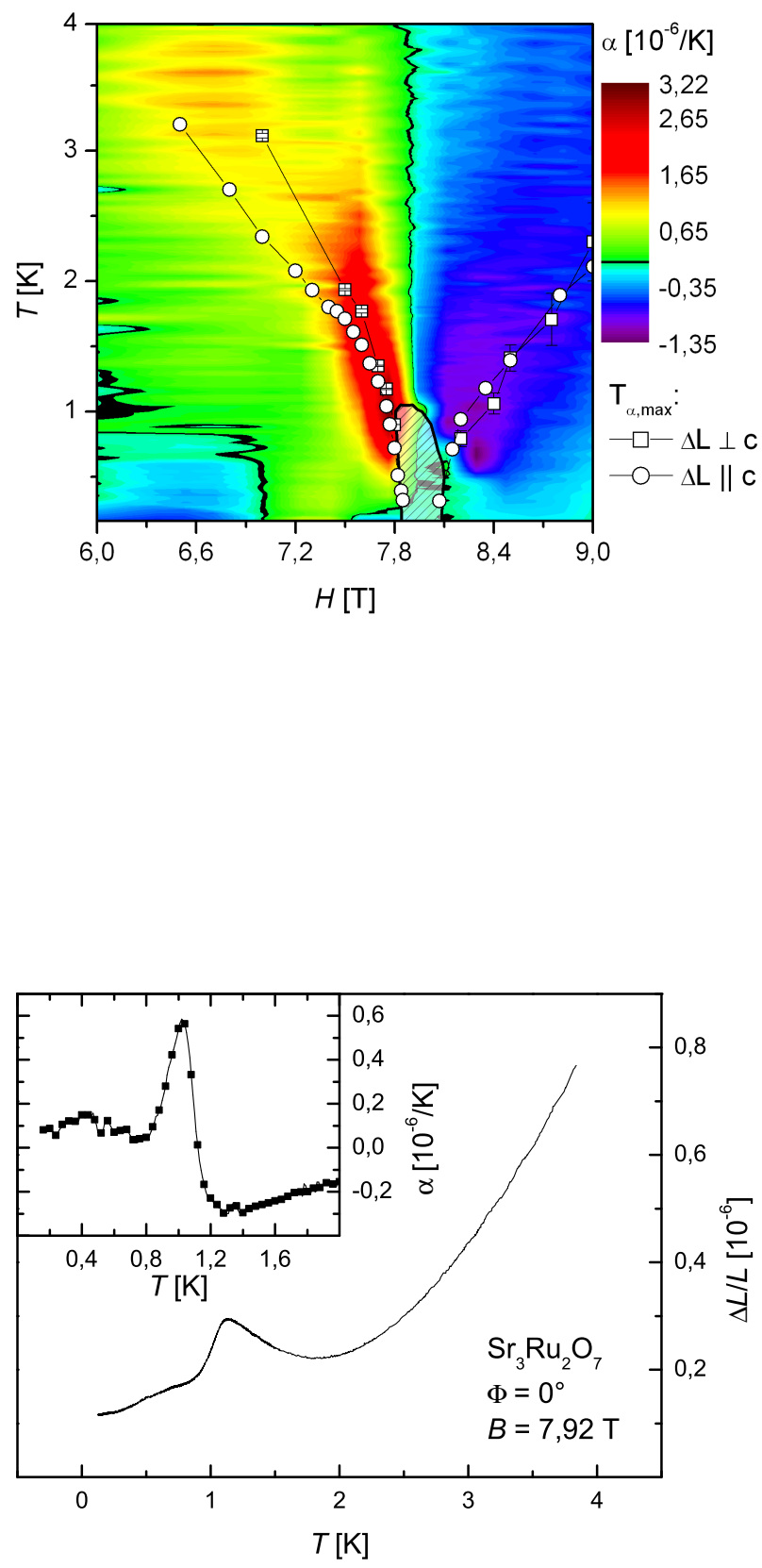

Abbildung 5.4: Übersicht über das Verhalten des kritischen Anteils von $\alpha$ in der $a b$-Ebene. Orte mit $\alpha=0$ sind in schwarzer Farbe codiert. $T_{\alpha, \max }(B)$, die Positionen maximaler bzw. minimaler thermischer Ausdehnung, verlaufen ähnlich wie in $c$-Richtung.

Abbildung 5.5: Beim „Dach“ der nematischen Phase zeigt sich eine sprunghafte Längenänderung von $\Delta L / L \approx$ $10^{-7}$. Die thermische Ausdehnung $\alpha$ (Inset) zeigt die Signatur eines Phasenübergangs zweiter Ordnung. 


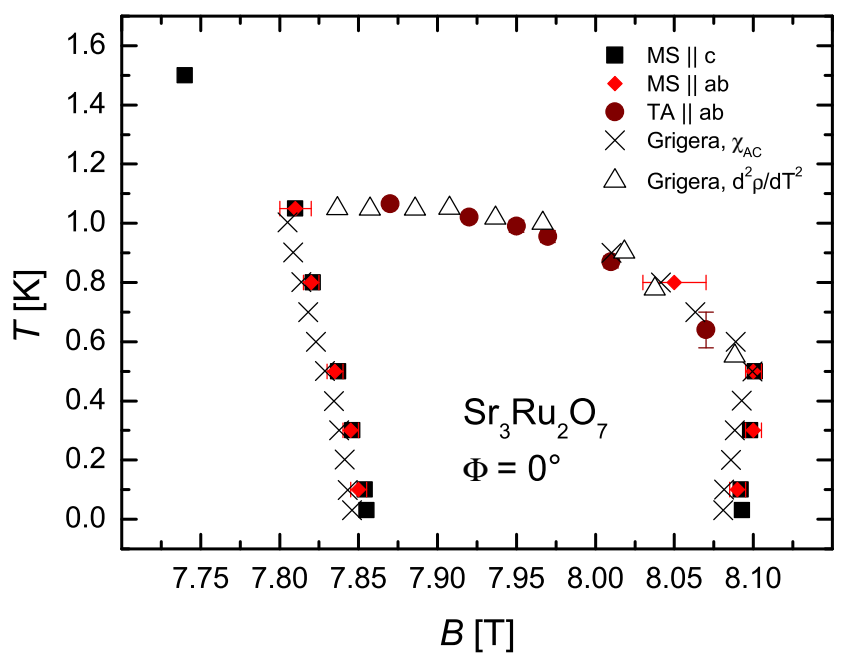

Abbildung 5.6: Aus Magnetostriktion und thermischer Ausdehnung gewonnenes Phasendiagramm. Es ergibt sich eine hervorragende Übereinstimmung mit den von Grigera Gri04 aus der Wechselfeldsuszeptibilität $\chi_{\mathrm{AC}}$ und dem spezifischen Widerstand $\mathrm{d}^{2} \varrho / \mathrm{d} T^{2}$ ermittelten Daten, vgl. Abb. 4.8 auf S. 44

Ausdehnungskoeffizient $\alpha$ wurde wie oben durch lineare Anpassungen über Intervalle von $80 \mathrm{mK}$ bestimmt; er zeigt einerseits einen Sprung und andererseits auch ein ausgeprägtes Maximum mit einer Halbwertsbreite von ca. $300 \mathrm{mK}$.

Die Orte der Maxima von $\alpha$ sind ebenfalls im Phasendiagramm in Abbildung 5.6 eingetragen. Wie schon für die Magnetostriktion ergibt sich eine hervorragende Übereinstimmung mit den bekannten Daten. Die Beobachtung einer klaren Signatur eines Phasenübergangs in der thermischen Ausdehnung in der Ebene ist besonders bemerkenswert, da parallel zur c-Richtung lediglich schwache Anomalien beim Eintritt in die nematische Phase auftreten Geg06b. Während die obere Phasengrenze („Dach“) zuerst nur in Transportmessungen beobachtet wurde, haben Rost et al. Ros09] kürzlich einen Sprung in der spezifischen Wärme gefunden. Dieser tritt allerdings bei einer Temperatur von $1,2 \mathrm{~K}$ auf und liegt damit deutlich höher als die Anomalien aller anderen Messungen. Möglicherweise wird hier ein Kristall von noch höherer Qualität als in früheren Veröffentlichungen verwendet, bei dem der Eintritt in die nematische Phase bereits bei einer höheren Temperatur erfolgt. Anderseits könnte die spezifische Wärme auch Anomalien aufweisen, die nicht direkt mit der oberen Phasengrenze in Verbindung stehen. In jedem Fall stellen die Messungen in dieser Arbeit eine der ersten deutliche Beobachtungen des Phasenübergangs in einer thermodynamischen Meßgröße dar.

Die Signatur von $\alpha$ zeigt eine beginnende Divergenz und $\Delta L$ einen, wenn auch verbreiterten, Sprung, was sich als Phasenübergang erster Ordnung interpretieren ließe. Da der Sprung aber nicht scharf ist, keine Hysterese beobachtet wird und außerdem ganz allgemein Phasenübergänge zweiter Ordnung im strengen ehrenfestschen Sinne selten sind, ist aber ein nicht mean-field-artiger Phasenübergang zweiter Ordnung wahrscheinlich. Auch wenn sich die Ordnung des Phasenübergangs nur aus der thermischen Ausdehnung nicht mit abschließender Sicherheit feststellen läßt, wird im folgen- 

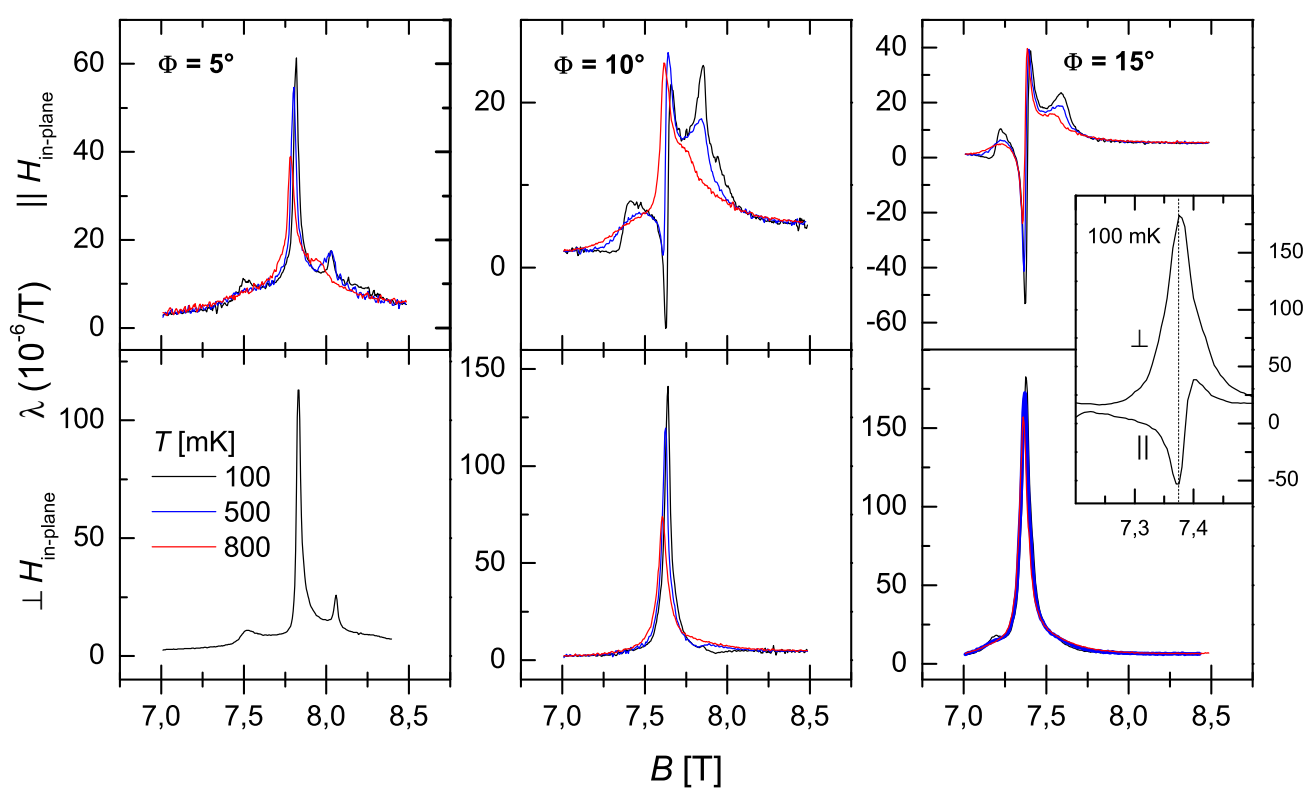

Abbildung 5.7: Magnetostriktion $\lambda$ in der Basalebene von $\operatorname{Sr}_{3} \mathrm{Ru}_{2} \mathrm{O}_{7}$ für um $\Phi=\Varangle(B, \mathrm{c})$ verkippte Felder, gemessen parallel (obere Zeile) bzw. senkrecht zu $H_{\text {in-plane }}$ (untere Zeile). Bei $\Phi=5^{\circ}$ ist $\lambda$ noch weitgehend isotrop. Für $\Phi \geq 10^{\circ}$ zeigt sich beim Eintritt in die nematische Phase eine Kontraktion parallel zum In-Plane-Feld, während sich die Probe in senkrechter Richtung ausdehnt.

den von einem Phasenübergang zweiter Ordnung ausgegangen. Dies ist auch konsistent mit der in der spezifischen Wärme gefundenen Signatur.

\subsubsection{Verkippte Felder}

Magnetostriktion Um die Position der metamagnetischen Übergänge und damit die Lage der nematischen Phase im $(B, T)$-Diagramm festzustellen, wurde jeweils zuerst die Magnetostriktion untersucht. Sie ist in Abbildung 5.7 für die zwei Meßrichtungen gezeigt. Für $\Phi=5^{\circ}$ ist $\lambda$ in der Ebene noch weitgehend isotrop: Die Signatur ist gegenüber dem Fall des unverkippten Feldes (Abbildung 5.2 rechts) nur wenig verändert, allerdings ist im Vergleich das Maximum beim metamagnetischen Hauptübergang für $\Delta L \| B_{\text {in-plane }}$ leicht verringert und für $\Delta L \perp B_{\text {in-plane }}$ entsprechend erhöht.

Für $\Phi=10^{\circ}$ ergibt sich ein deutlich unterschiedliches Verhalten: Während $\lambda_{\perp}$ am Hauptübergang nach wie vor positiv ist, wird $\lambda_{\|}$beim gleichen Feld negativ. Diese stark anisotrope Längenänderung entspricht einer Verzerrung der Probe in der Ebene beim Eintritt in die nematische Phase. Der Effekt ist temperaturabhängig: bei $T=800 \mathrm{mK}$ hat $\lambda_{\|}$wieder ein positives Vorzeichen, bleibt aber einen Faktor 2 kleiner als $\lambda_{\perp}$, so daß man nach wie vor von einer Verzerrung sprechen kann. Der zweite Übergang ist in $\lambda_{\perp}$ nur bei der tiefsten gemessenen Temperatur aufzulösen, dafür zeigt sich nun in 
$\lambda_{\|}$ein deutlicher positiver Längensprung. Auch beim zweiten Übergang findet man also eine anisotrope Längenänderung, allerdings mit entgegengesetzter Tendenz: der beim Eintritt in die nematische Phase eingenommene Verzerrungszustand wird beim Verlassen zumindest qualitativ, wenn auch nicht quantitativ, wieder abgebaut.

Das beschriebene Verzerrungsverhalten verstetigt sich für einen größeren Verkippungswinkel von $\Phi=15^{\circ}$ : Beim ersten Übergang wird das Minimum in $\lambda_{\|}$noch deutlich kleiner und behält auch bei der höchsten gemessenen Temperatur sein negatives Vorzeichen.

Interessanterweise folgt unmittelbar auf die Kontraktion in $\Delta L_{\|}$wieder ein Maximum in $\lambda_{\|}$. Wie die detaillierte Auftragung im Inset zu Abbildung 5.7 zeigt, wird es aber im Gegensatz zum Minimum nicht von einer Signatur in $\lambda_{\perp}$ begleitet. Für die Bestimmung der Grenzlinien im in Abbildung 5.10 gezeigten Phasendiagramm werden deshalb das Minimum in $\lambda_{\|}$und das Maximum in $\lambda_{\perp}$ herangezogen. Da aus den Messungen nicht mit Sicherheit zwischen Phasenübergängen erster und zweiter Ordnung unterschieden werden kann, sind die kritischen Endpunkte der metamagnetischen Übergänge, wie sie für unverkippte Felder der Abbildung 4.8 entnommen werden können, nicht eingezeichnet.

Bei Betrachtung des Phasendiagramms zeigt sich, daß sich mit steigendem Verkippungswinkel $\Phi$ die Lage der Übergänge zu kleineren Magnetfeldern verschiebt. Dies ist qualitativ zu erwarten, da das metamagnetische kritische Feld für $B \| a b$ deutlich kleiner ist und sich dazwischen monoton verhält. Zum genaueren Vergleich sind in Abbildung 5.10 auch Datenpunkte aus der Arbeit von RAGHU et al. Rag09] eingetragen, die aus Messungen von Suszeptibilität und spezifischem Widerstand die Lage der Phasengrenzen bei $T=100 \mathrm{mK}$ als Funktion des Winkels bestimmt haben.

Thermische Ausdehnung Nachdem nun für die jeweiligen Winkel $\Phi$ die Lage der metamagnetischen Übergänge und damit der nematischen Phase im $(B, T)$-Diagramm bekannt ist, soll die thermische Ausdehnung bei konstantem Magnetfeld betrachtet werden. Hierfür wurden jeweils 3 Felder innerhalb der Phase und zwei Felder außerhalb der Phase ausgewählt und in einem Temperaturbereich von $120 \mathrm{mK} \leq T \leq 4 \mathrm{~K}$ vermessen. Die Abbildung 5.8 gibt einen Überblick über die so erhaltenen Meßdaten.

Für $\Phi=5^{\circ}$ zeigen bei höheren Temperaturen die Kurven $\Delta L_{\|}$und $\Delta L_{\perp}$ fast genau aufeinander. Zum Vergleich ist zusätzlich die entsprechende ${ }^{2}$ Messung für $\Phi=0^{\circ}$ bei $B=7.92 \mathrm{~T}$ mit aufgetragen, die bei hohen Temperaturen das gleiche Verhalten zeigt. Beim Eintritt in die nematische Phase zeigt sich in beiden Meßrichtungen eine deutliche spontane Längenänderung, wie sie auch schon für $\Phi=0^{\circ}$ beobachtet wurde. Innerhalb der nematischen Phase bei $T<1 \mathrm{~K}$ beginnt sich eine Anisotropie zu entwickeln: die Änderung in $\Delta L_{\perp}$ wird klein, während in $\Delta L_{\|}$beim Abkühlen eine deutlich stärkere Kontraktion erfolgt.

Für $\Phi=10^{\circ}$ verstärkt sich diese Anisotropie deutlich: Die relative Änderung von

\footnotetext{
${ }^{2}$ Die Felder sind so gewählt, daß die relative Position zum metamagnetischen Übergang gleich bleibt, vgl. Abbildung 5.10 .
} 


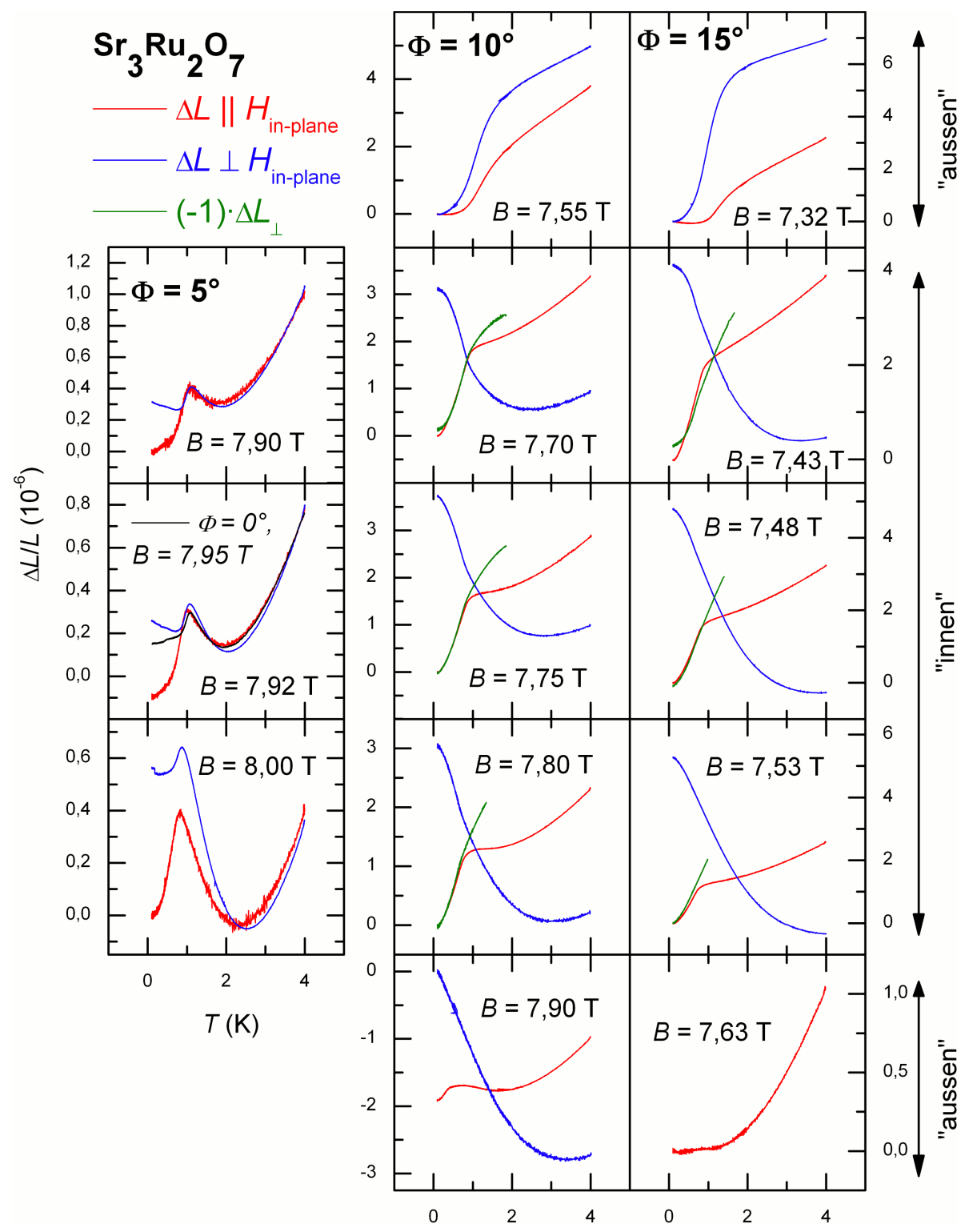

Abbildung 5.8: Thermische Ausdehnung $\Delta L(T) / L$ in der Basalebene von $\mathrm{Sr}_{3} \mathrm{Ru}_{2} \mathrm{O}_{7}$ für um $\Phi=\Varangle(B, c)$ verkippte Felder. Bei Messungen mit Magnetfeldern innerhalb (zweite bis vierte Zeile) des nematischen Bereichs findet man $\Delta L_{\|}+\Delta L_{\perp}=0$ für $\Phi \geq 10^{\circ}$, was außerhalb (erste und letzte Zeile) nicht erfüllt ist. Alle Kurven sind in vertikaler Richtung willkürlich verschoben. 
$L_{\|}$in der nematischen Phase wird gegenüber $\Phi=5^{\circ}$ um einen Faktor $3-4$ größer. In $\Delta L_{\perp}$ findet beim Abkühlen nun sogar eine Expansion statt. Damit zeigt auch die thermische Ausdehnung die Existenz des bereits in der Magnetostriktion gefundenen Verzerrungszustands innerhalb der nematischen Phase, wobei in beiden Meßgrößen übereinstimmend eine Kontraktion in $\Delta L_{\|}$und eine Expansion in $\Delta L_{\perp}$ auftritt.

Der für $\Phi=0^{\circ}$ und $\Phi=5^{\circ}$ (hier in beiden gemessenen Richtungen) gefundene sprungartige Längenänderung am „Dach“ läßt sich für $\Phi=10^{\circ}$ nicht mehr feststellen, sie hat sich vielmehr zu einem deutlichen Knick in $\Delta L_{\|}$entwickelt, wohingegen in $\Delta L_{\perp}$ keine Signatur des Phasenübergangs mehr zu sehen ist.

Ein genauerer Vergleich der thermischen Ausdehnung für $\Delta L \| B_{\text {in-plane }}$ und $\Delta L \perp$

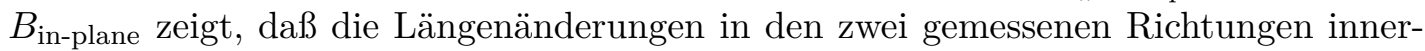
halb der Phase nicht unabhängig voneinander sind: Die Multiplikation von $\Delta L_{\perp}$ mit dem Faktor $(-1)$ ergibt eine Kurve, die bei vielen Feldern mit äußerster Genauigkeit mit $\Delta L_{\|}$übereinstimmt und erst bei Temperaturen oberhalb der Phasengrenzlinie abweicht (grüne Kurven in Abbildung 5.8). Zwar ergeben sich für einzelne Magnetfelder auch kleinere Abweichungen innerhalb der Phase, die aber unbedeutend erscheinen im Vergleich mit Feldern außerhalb der Phase, wo ein Zusammenhang zwischen den Kurven nicht zu beobachten ist. Die thermische Ausdehnung gehorcht also innerhalb der nematischen Phase der Beziehung

$$
\Delta L_{\|}(T)+\Delta L_{\perp}(T)=0
$$

was als eine gleichförmige, also den Flächeninhalt erhaltende, Verzerrung bezeichnet werden kann.

Für einen höheren Verkippungswinkel $\Phi=15^{\circ}$ bleibt die absolute Längenänderung innerhalb der Phase mit (je nach Feld) $\Delta L / L=1 \ldots 2 \cdot 10^{-6}$ unverändert und 5.1 gilt weiterhin. Lediglich $\Delta L_{\perp}$ ist für hohe Temperaturen größer geworden. Wie bereits in den Magnetostriktionsdaten ist also bei $\Phi=5^{\circ}$ eine beginnende Anisotropie zu beobachten, die bei $10^{\circ}$ voll einsetzt und bei $15^{\circ}$ gesättigt ist.

In Abbildung 5.9 ist der aus den besprochenen Längendaten berechnete thermische Ausdehnungskoeffizient $\alpha$ aufgetragen. Auch hier ist bei tiefen Temperaturen die gleichförmige Verzerrung $\alpha_{\|}+\alpha_{\perp}=0$ erkennbar. $\alpha_{\|}$zeigt am Phasenübergang einen scharfen Sprung von $2-3 \cdot 10^{-6} \mathrm{~K}^{-1}$ auf sehr kleine Werte. Die Spitze, die für $\Phi=0^{\circ}$ den Sprung in $\alpha$ überlagerte, tritt nicht mehr auf. In $\alpha_{\perp}$ sind für $\Phi=10^{\circ}$ noch Ansätze des Sprungs zu sehen, für $\Phi=15^{\circ}$ allerdings nicht mehr auszumachen. Das Verhalten bei tiefen Temperaturen ist linear in $T$ und gehorcht damit der für eine Fermiflüssigkeit erwarteten Abhängigkeit $\alpha \propto T$. Dies ist konsistent mit den dHvA-Messungen (Abschnitt 4.4.1), wo ebenfalls Fermiflüssigkeitsverhalten gefunden wurde.

Die Anomalien in der thermischen Ausdehnung können nun zur Bestimmung der oberen Phasengrenzlinie („Dach“) verwendet werden. Für $\Phi=5^{\circ}$ werden die Orte der Maxima in $\alpha$ eingetragen, während für größere Winkel eine gleiche-Flächen-Konstruktion (sog. Zwickel-Abgleich) zum Einsatz kommt. Dabei wird die real gemessene, durch Fluktuationseffekte verbreiterte Kurve $\alpha(T)$ durch einen idealisierten scharfen Sprung 

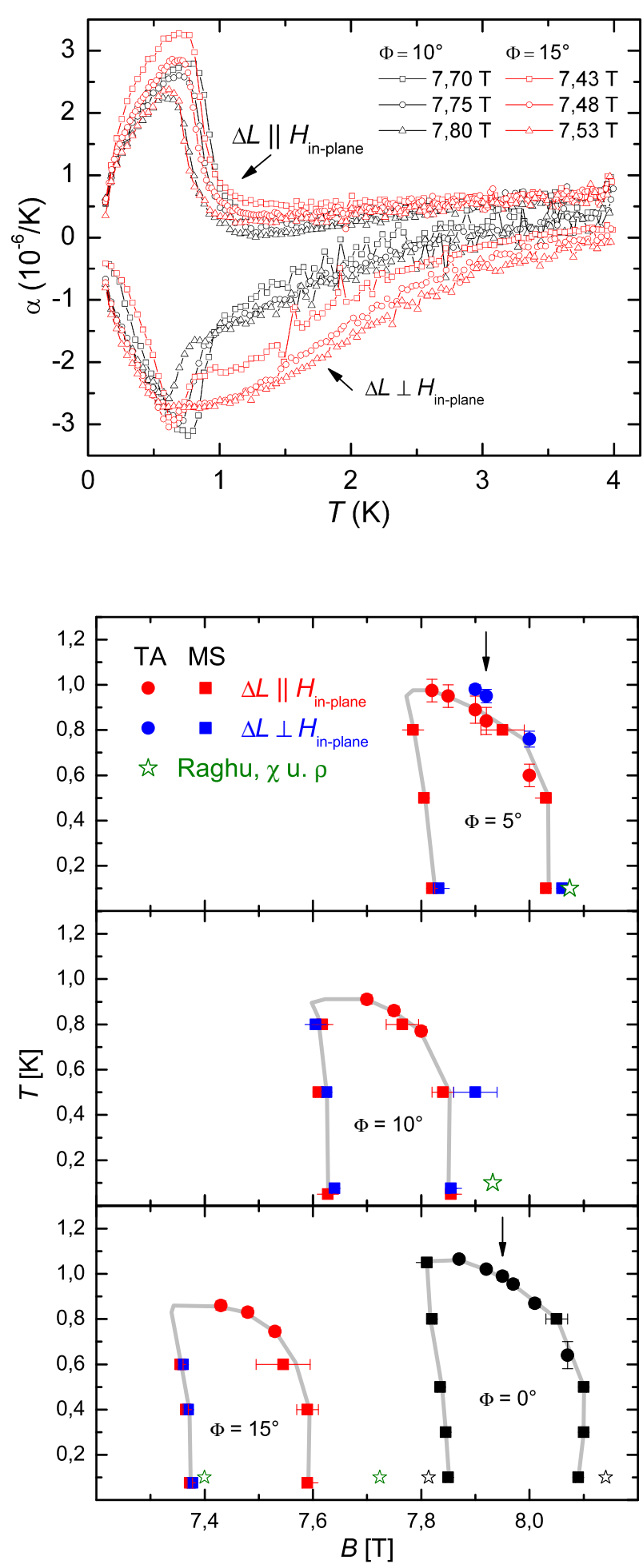

Abbildung 5.9: Thermischer Ausdehnungskoeffizient $\alpha$ für große Verkippungswinkel $\Phi$ und Felder innerhalb der nematischen Phase.
Abbildung 5.10: Phasendiagramm für um den Winkel $\Phi$ gegen die c-Richtung verkippte Magnetfelder. Die nematische Phase verschiebt sich mit steigendem Winkel $\mathrm{zu}$ kleineren Feldern. Die Felder, bei denen die in Abb. 5.8 gezeigte thermische Ausdehnung gemessen wurde (rote Kreise), wurden so gewählt, daß sie die gleiche relative Position in Bezug auf die Phasengrenzen haben. Die Pfeile markieren die Felder, für die in Abb. 5.8 die thermische Ausdehnung für $0^{\circ}$ und $5^{\circ}$ verglichen wird. 


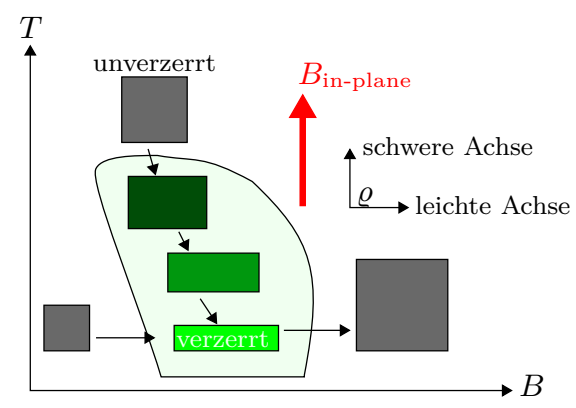

\begin{abstract}
Abbildung 5.11: Qualitative Zusammenfassung der Meßergebnisse. Die Richtungen der Expansion und Kontraktion relativ zu $B_{\text {in-plane }}$ lassen sich in Zusammenhang bringen mit der leichten und schweren Achse für den elektronischen Transport $(\varrho)$.
\end{abstract}

bei $T=T_{\mathrm{c}}$ ersetzt. Praktisch läßt sich dies durch Beschreibung mit einer SigmoidFunktion

$$
s(x)=\frac{1}{1+\exp (-x)}
$$

realisieren und man erhält

$$
\begin{aligned}
\alpha(T) & =a+(b-a) \frac{1}{1+\exp \left(-g\left(T-T_{\mathrm{c}}\right)\right)} \\
\Rightarrow \Delta L(T) & =\int \alpha(T) \mathrm{d} T=a+(b-a) \frac{1}{g} \log \left(1+\exp \left(g\left(T-T_{\mathrm{c}}\right)\right)\right)+c .
\end{aligned}
$$

Da (5.2) die Eigenschaft $s(x)=1-s(-x)$ besitzt, beschreibt (5.3) einen um $T_{\mathrm{c}}$ symmetrischen Sprung in $\alpha$ der Höhe $b-a$ mit $1 / g$ als Maß für die Breite. Die Funktion (5.4) kann direkt an die gemessenen Längendaten angepaßt werden, wodurch der fehlerbehaftete Ableitungsschritt wegfällt, und man erhält $T_{\mathrm{c}}$ als Anpassungsparameter.

Mit den erhaltenen Übergangstemperaturen kann nun das Phasendiagramm in Abbildung 5.10 vervollständigt werden.

\title{
5.2 Diskussion
}

Die im vorangegangenen Abschnitt vorgestellten dilatometrischen Untersuchungen in $\mathrm{Sr}_{3} \mathrm{Ru}_{2} \mathrm{O}_{7}$ haben zwei wesentliche Resultate erbracht: Dies ist zum einen die gleichförmige Verzerrung der Basalebene innerhalb der nematischen Phase, welche im Folgenden im Rahmen eines kürzlich veröffentlichten Modells diskutiert werden soll. Zum anderen wurde in $\alpha$ eine klare thermodynamische Signatur für die obere Phasengrenzlinie gefunden, die in der Diskussion näher untersucht wird.

\subsubsection{Verzerrung}

Die Ergebnisse aus Magnetostriktion und thermischer Ausdehnung liefern eindeutige Hinweise auf die Existenz eines verzerrten Zustands in der nematischen Phase. In Abbildung 5.11 ist das gefundene Verhalten schematisch illustriert: Sowohl in $T$ - als auch in $B$-Richtung kommt es bei Eintritt in die Phase zu einer Längenkontraktion parallel 
zu $B_{\text {in-plane }}$ und zu einer Expansion in der senkrechten Richtung. Die Zustände außerhalb der Phase sind als „unverzerrt" skizziert und beschrieben. Da im Experiment nur Längenänderungen gemessenen werden, ist dies nicht im strengen Sinne korrekt; insbesondere wird in der Magnetostriktion beim ersten Übergang eine Verzerrung aufgebaut, die beim zweiten Übergang zwar tendenziell wieder abgebaut, aber nicht in voller Größe kompensiert wird. ${ }^{3}$ Die Skizze ist deshalb so zu verstehen, daß innerhalb der Phase eine stärkere Verzerrung vorliegt als außerhalb, was von den Meßdaten voll untermauert wird. Die Verzerrung ist von der Größenordnung $10^{-7}$ für $B \| \mathrm{c}$ und $10^{-6}$ für verkippte Felder. Dies ist unterhalb der instrumentellen Auflösung von $10^{-5}$ von Neutronenbeugungsexperimenten, in denen keine Gitterverzerrung festgestellt werden konnte Bor07.

Es kann nun ein Vergleich mit dem in Abschnitt 4.4 beschriebenen Widerstandsexperiment vorgenommen werden, um die dort gefundene leichte und schwere Achse für den Stromfluß mit den Richtungen der Kontraktion bzw. Ausdehnung in Verbindung zu bringen. Es zeigt sich (Abbildung 5.11), daß die Ausdehnung in Richtung der leichten Achse und die Kontraktion in Richtung der schweren Achse erfolgt. Damit ist eine zusätzliche Randbedingung für Modelle der nematischen Phase gegeben.

Modell der partiellen orbitalen Ordnung LEE und WU LW10 haben kürzlich eine Theorie für $\mathrm{Sr}_{3} \mathrm{Ru}_{2} \mathrm{O}_{7}$ veröffentlicht, die auf einem Tight-Binding-Ansatz aus $d_{x y^{-}}$, $d_{x z^{-}}$und $d_{y z}$-Orbitalen beruht und außerdem die Spin-Bahn-Wechselwirkung berücksichtigt. Das Modell reproduziert die bekannte Fermifläche in guter Näherung. Die Besetzung der Zustände wird im Rahmen eines Hubbard-Modells behandelt, das auf einem Gitterplatz Wechselwirkungen zwischen verschiedenen Orbitalen berücksichtigt. Der nematische Ordnungsparameter $N=2\left(n^{y z}-n^{x z}\right)$ ist dann, wie auch schon im Modell von RAGHU et al. Rag09], durch den Besetzungsunterschied zwischen den Quasi-1D-Bändern $d_{y z}$ und $d_{x z}$ gegeben.

In den Rechnungen zeigen sich drei metamagnetische Übergänge bei $16 \mathrm{~T}, 26 \mathrm{~T}$ und $29 \mathrm{~T}$, womit das Verhalten des realen Systems zumindest der Größenordnung nach wiedergegeben wird. Zwischen dem zweiten und dritten Übergang kommen Teile der Fermifläche in der Nähe der X-Punkte in die Nähe der van-Hove-Singularität und der Ordnungsparameter wird endlich mit einem Wert von $N \approx 0,02$. Das heißt, daß ca. $1 \%$ der Elektronen vom $d_{x z^{-}}$ins $d_{y z}$-Band umverteilt werden, man kann deshalb von einer partiellen orbitalen Ordnung sprechen. Mit dieser Ordnung geht eine leichte Verzerrung der Fermifläche in der Nähe der X-Punkte einher, wo sich eine van-Hove-Singularität in der Zustandsdichte befindet, vgl. Abschnitt 4.1. Die hohe Zustandsdichte impliziert ein schmales Band und damit eine kleine Fermigeschwindigkeit $v_{\mathrm{F}}=\partial \epsilon /\left.\partial k\right|_{k_{\mathrm{F}}}$, weswegen die Elektronen in den nematisch verzerrten Bereichen der Fermifläche nur wenig zum Transport beitragen. Der erhöhte spezifische Widerstand für das unverkippte Feld ist

\footnotetext{
${ }^{3}$ Eine vollständige Kompensation ist allerdings auch nicht zu erwarten, da das In-Plane-Feld einen expliziten Bruch der Rotationssymmetrie in der Ebene darstellt und deshalb auch außerhalb der spontan symmetriegebrochenen Phase keine isotrope Magnetostriktion angenommen werden kann.
} 


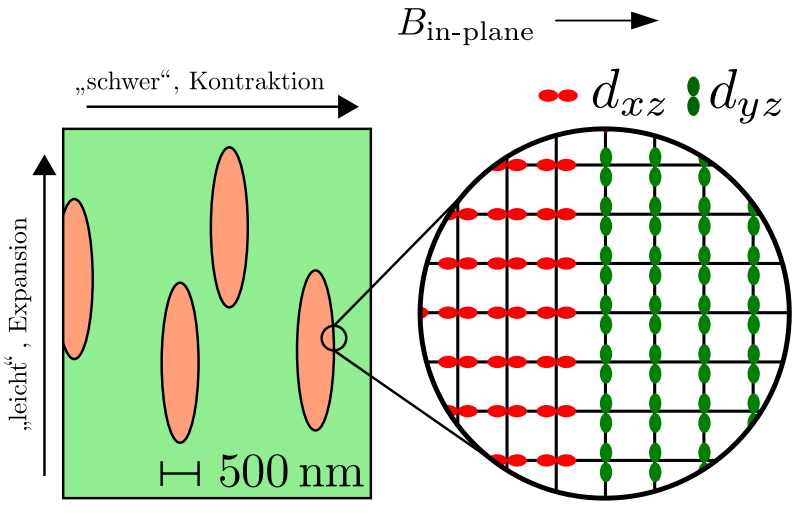

Abbildung 5.12: Von LEE und Wu LW10 vorgeschlagenes Modell zur partiellen orbitalen Ordnung. In der nematischen Phase gibt es in den $d_{x z^{-}}$und $d_{y z}$-Bändern einen Besetzungsunterschied von ca. $1 \%$, der hier stark übertrieben in einem lokalisierten Bild dargestellt ist. $B_{\text {in-plane hebt }}$ die Entartung der Orbitale auf und bevorzugt Domänen mit einer erhöhten Besetzung von $d_{y z}$.

deshalb nur im Rahmen des Domänenmodells zu verstehen. Da $d_{x z}$ und $d_{y z}$ ohne ein In-Plane-Feld entartet sind, sollte $N$ gleichermaßen positive wie negative Werte annehmen können, was einer orbitalen Ordnung in $x$ - bzw. $y$-Richtung entspricht. Die zwei möglichen Varianten sollen im folgenden als $N^{+}$und $N^{-}$bezeichnet werden. Wenn geringe Unordnung in der Probe lokal eine der beiden Varianten (z. B. durch lokale Kristallfeldaufspaltung) bevorzugt, kann dies zur Bildung einer Domänenstruktur führen, die den erhöhten Restwiderstand als Streuung an Domänenwänden erklärt. Da beide Varianten im Mittel gleich häufig sind, ist das gemessene Signal isotrop.

Zum Vergleich mit den in dieser Arbeit vorgestellten Meßergebnissen muß nun geklärt werden, welche Kopplung ans Gitter für das Modell der partiellen orbitalen Ordnung zu erwarteten ist. Abbildung 4.2 zeigt, daß die $\gamma_{2}$-Fermiteilfläche, welche in der Nähe der van-Hove-Singularität liegt, unbesetzte Zustände einschließt. Es handelt sich also um lochartige Zustände, deren Fermienergie nahe an der oberen Bandkante liegt. Solche Zustände haben antibindenden Charakter [Wu10], wie er sich bspw. am Wasserstoffmolekül veranschaulichen läßt. Dort sinkt die Energieaufspaltung zwischen bindendem und antibindendem Zustand mit steigendem Ionenabstand. Die erhöhte Besetzung eines antibindenden Zustands sollte also aus energetischen Gründen zu einer Expansion des Gitters in der entsprechenden Richtung führen.

In der thermischen Ausdehnung für unverkippte Felder wird mit Eintritt in die nematische Phase stets eine Kontraktion in der Meßrichtung beobachtet. Das bedeutet aber, daß die zwei Varianten von Domänen nicht mehr gleichverteilt sein können, da andernfalls im Mittel über alle Domänen keine Verzerrung zu beobachten wäre. Das obige Argument zur Kopplung ans Gitter kann allerdings auch in umgekehrter Richtung geführt werden: ein uniaxialer Druck in der Ebene, der zu einer Kompression entlang einer Kristallachse führt, sollte den entsprechenden antibindenden Zustand energetisch anheben. Ein solcher Druck wird nun gerade, wie in Abschnitt 3.2.4 ausgeführt, durch die Federkraft des Dilatometers auf die Probe ausgeübt. Wird also z. B. $\Delta L$ in der $x$-Richtung gemessen, so wird dadurch das $d_{y z}$-Band energetisch begünstigt. Es bildet sich ein leichter Überschuß von $N^{+}$-Domänen und es kommt zu einer Kontraktion in der $x$-Richtung. Damit ist erklärt, warum im unverkippten Feld beim Eintritt in die nematische Phase stets eine Kontraktion beobachtet wird: das System 
bevorzugt eine Variantenverteilung, um damit dem uniaxialen Druck nachzugeben.

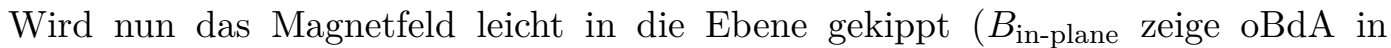
die $x$-Richtung), so muß im Hamiltonoperator zusätzlich ein Zeemanterm der Form $H_{\text {in-plane }}=-\mu_{\mathrm{B}} B \cos (\Phi) \hat{L_{x}}$ berücksichtigt werden, welcher die Entartung der $d_{x z^{-}}$und $d_{y z}$-Zustände aufhebt: dadurch wird $d_{y z}$ energetisch abgesenkt und damit die Variante $N^{+}$bevorzugt, was wiederum eine Kontraktion in $x$-Richtung ergeben sollte. In der Tat beobachtet man für verkippte Felder beim Eintritt in die nematische Phase eine deutlichere Kontraktion parallel zum In-Plane-Feld. Das verkippte Feld verstärkt also

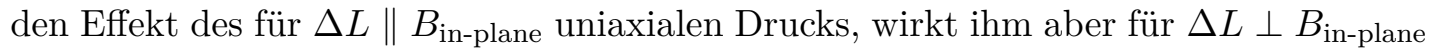
entgegen, was beim Vergleich der thermischen Ausdehnung für $\Phi=0^{\circ}$ und $\Phi=5^{\circ}$ deutlich wird. Die explizite Symmetriebrechung durch den uniaxialen Druck bewirkt offensichtlich nur eine kleine Änderung der Variantenverteilung, da der Verzerrungszustand sich mit zunehmendem Kippwinkel weiter verstärkt. Bei $\Phi=15^{\circ}$ erscheint die Verzerrung allerdings gesättigt, so daß hier von der fast ausschließlichen Existenz der bevorzugten Domänenvariante auszugehen ist.

Die Aufhebung der Entartung durch ein verkipptes Magnetfeld bleibt nicht ohne Auswirkungen auf die Form der Domänen, da eine Domänenwand im lokalisierten Bild mit dem Aufbrechen von Bindungen einhergeht. Sind bei einem In-Plane-Feld in $x$-Richtung die $d_{x z}$-Bindungen energetisch angehoben, dann sollte ein Aufbrechen derselben weniger Energie kosten, während $d_{y z}$-Bindungen bevorzugt erhalten bleiben. Domänenwände richten sich demnach bevorzugt in $y$-Richtung aus, was zu einer länglichen Form der Domänen führt, wie sie in Abbildung 5.12 skizziert ist. Die aus dHvA-Messungen bekannte Längenskala von $500 \mathrm{~nm}$ entspricht dabei der kurzen Domänenachse.

Die skizzierte mesoskopische Struktur sollte ein deutlich anisotropes Transportverhalten zeigen, da ein Elektron in der Richtung parallel zu $B_{\text {in-plane }}$ auf viele Domänenwände trifft und damit häufig gestreut wird. In der senkrechten Richtung ist die freie Weglänge dagegen viel größer. Auch Minoritätsdomänen, die nur einige Prozent der Probe ausmachen, könnten also noch für eine starke Widerstandsanisotropie sorgen, während das Ausdehnungsverhalten fast vollständig von den Majoritätsdomänen bestimmt wird. Es besteht deshalb kein Widerspruch zwischen der in dieser Arbeit festgestellten Sättigung der Gitterverzerrung bei $10^{\circ}-15^{\circ}$ und den Widerstandsexperimenten, die bei diesen Winkeln noch Anisotropie zeigen. In der Tat beobachtet man im Widerstand nematische Eigenschaften noch bis zur Bifurkation des metamagnetischen Übergangs bei $\Phi=35^{\circ}$ [Rag09 . Möglicherweise sind Minoritätsdomänen durch lokale Defekte verankert und bleiben deshalb auch bei starken In-Plane-Feldern erhalten.

Temperaturabhängigkeit Nachdem also die gefundene Verzerrung qualitativ gut im Modell der partiellen orbitalen Ordnung verstanden werden kann, stellt sich nun die Frage, ob sich damit auch die Temperaturabhängigkeit, insbesondere das Fermiflüssigkeitsverhalten und die gleichförmige Verzerrung, erklären läßt. Ein möglicher Ansatz wäre der folgende: Der Besetzungsunterschied zwischen den $d_{x z^{-}}$und $d_{y z}$-Bändern ist 
klein, so daß für beide Richtungen die gleiche Kopplung der Besetzungszahlen an das Gitter,

$$
\Delta L_{x}=f\left(\Delta n^{x z}\right), \quad \Delta L_{y}=f\left(\Delta n^{y z}\right),
$$

mit einer ungeraden Funktion $f$ erwartet werden kann. Mit steigender Temperatur werden nun in der nematischen Phase Ladungsträger in das energetisch ungünstigere Band angeregt, so daß der Ordnungsparameter $N$ kleiner wird, bis die nematische Ordnung schließlich am „Dach“ der Phase verschwindet. Genau dieses Verhalten wird auch experimentell im phänomenologischen Ordnungsparameter Gleichung (4.3) gefunden. Kann nun Ladungserhaltung zwischen den $d_{x z^{-}}$und $d_{y z}$-Bändern vorausgesetzt werden, d. h. $\Delta n^{x z}+\Delta n^{y z}=0$, so folgt aus Gleichung (5.5) $\Delta L_{x}+\Delta L_{y}=0$, womit die in dieser Arbeit gefundene gleichförmige Verzerrung (5.1) erklärt wäre.

Gerade die Voraussetzung der Ladungserhaltung ist aber möglicherweise problematisch, da neben $d_{x z}$ und $d_{y z}$ noch weitere Bänder zur elektronischen Struktur beitragen. Insbesondere $d_{x y}$ stellt in Rechnungen ein Teilchenreservoir dar, welches das chemische Potential konstant hält [Rag09].

Der soeben besprochene Ansatz geht davon aus, daß die thermische Ausdehnung durch die Temperaturabhängigkeit des Ordnungsparameters bestimmt wird. Andererseits ist auch bei einem konstanten Ordnungsparameter thermische Ausdehnung des Elektronensystems zu erwarten, was im folgenden diskutiert werden soll:

In Übereinstimmung mit anderen Arbeiten wurde in der nematischen Phase Fermiflüssigkeitsverhalten gefunden mit $\Delta L / L \propto T^{2}$ bzw. $\alpha \propto T$. Überraschenderweise besitzt $\alpha$ allerdings in den zwei gemessenen Richtungen ein unterschiedliches Vorzeichen. Für eine Fermiflüssigkeit ist die spezifische Wärme linear in der Temperatur, $c=\gamma T$, wobei der Sommerfeldkoeffizient $\gamma$ nach Gleichung (2.1) proportional zur elektronischen Zustandsdichte an der Fermikante ist. Daraus ergibt sich die Entropie als $S=\int c / T \mathrm{~d} T=\gamma T+$ const. $^{4}$ und die thermische Ausdehnung wird damit zu

$$
\alpha=-\frac{1}{V}\left(\frac{\partial S}{\partial p}\right)_{T}=-\frac{1}{V} T \frac{\partial \gamma}{\partial p} \propto-T \frac{\partial D\left(E_{\mathrm{F}}\right)}{\partial p} .
$$

Die Bänder $d_{x z}$ und $d_{y z}$ sind quasi-eindimensional, es ist daher plausibel, daß die thermische Ausdehnung in der $x$ - bzw. $y$-Richtung ausschließlich durch eins der zwei Bänder bestimmt wird. Das Vorzeichen von $\alpha$ in der nematischen Phase mißt also in den zwei Richtungen die uniaxiale Druckabhängigkeit der Zustandsdichte. Für das

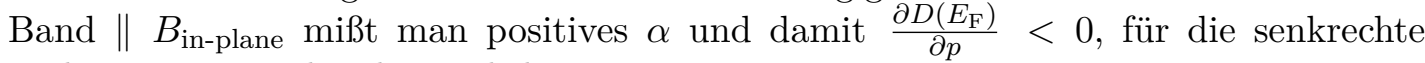
Richtung entsprechend umgekehrt.

Betrachtet man zum Vergleich ein einfaches Metall im Rahmen einer Tight-BindingNäherung, so steigt die Bandbreite mit sinkender Gitterkonstante. Dadurch wird die Zustandsdichte kleiner, so daß $\mathrm{d} D\left(E_{\mathrm{F}}\right) / \mathrm{d} p<0$. Das einfache Metall besitzt somit eine (isotrope) positive thermische Ausdehnung. Wie im Fall von $\mathrm{Sr}_{3} \mathrm{Ru}_{2} \mathrm{O}_{7}$ je nach Richtung unterschiedliche Vorzeichen von $\mathrm{d} D\left(E_{\mathrm{F}}\right) / \mathrm{d} p$ entstehen, ist in einem anschaulichen

\footnotetext{
${ }^{4}$ In einer Fermiflüssigkeit ist also die Entropie bis auf eine Konstante gleich der spezifischen Wärme.
} 
Modell weniger leicht zu verstehen. Besonders das Vorzeichen hängt von mikroskopischen Details der jeweiligen Bandstrukturrechnung ab und kann von den gegenwärtigen Modellen nicht ohne weiteres vorhergesagt werden Kiv11. Deshalb kann an dieser Stelle nur festgehalten werden: Falls die nematische Phase durch die FF-Theorie korrekt beschrieben wird, zeichnet sie sich durch eine stark anisotrope Druckabhängigkeit der Zustandsdichte aus.

Nachdem nun zwei mögliche Beiträge zur thermischen Ausdehnung diskutiert wurden, kann abschließend überlegt werden, welcher der zwei Effekte wann wichtig ist. Der Ordnungsparameter eines klassischen kontinuierlichen Phasenübergangs variiert für $t<0$ wie $|t|^{\beta}$ mit $\beta<1$ (vgl. Tabelle 2.1). Die Weisssche Molekularfeldtheorie des Ferromagneten Gol92 liefert z. B. $\beta=\frac{1}{2}$, die Magnetisierung verläuft also wurzelförmig und ist damit bei tiefen Temperaturen näherungsweise konstant, während sie ihre stärkste Änderung in der Nähe der kritischen Temperatur hat. Wenn hier ein ähnliches Verhalten vorausgesetzt werden kann (vgl. hierzu die Diskussion des Phasenübergangs weiter unten), so würden die zwei angesprochenen Effekte beide wie folgt in die thermische Ausdehnung eingehen:

Bei tiefen Temperaturen ist der Ordnungsparameter $n$ näherungsweise konstant und die FF-artige thermische Ausdehnung wird durch den Elektronenimpuls bestimmt. Bei höheren Temperaturen wird $n$ dagegen kleiner und der Verzerrungszustand damit abgebaut. Es kommt also bereits unterhalb der kritischen Temperatur zu Abweichungen vom FF-Verhalten. Bei Betrachtung von Abbildung 5.9 zeigt sich in der Tat, daß die Kurven $\alpha(T)$ noch vor dem eigentlichen Sprung abgerundet werden. Das vorgeschlagene Szenario erscheint damit plausibel.

\subsubsection{Quantenkritischer Einfluß}

Das bis jetzt diskutierte Modell kann die nematischen Eigenschaften mit großem Erfolg aus der Bandstruktur von $\mathrm{Sr}_{3} \mathrm{Ru}_{2} \mathrm{O}_{7}$ erklären, berücksichtigt aber noch nicht den Einfluß (quanten-)kritischer Fluktuationen. Während die thermische Ausdehnung im Rahmen einer Hertz-Millis-Theorie-behandelt wurde, in die insbesondere die Dimensionalität des Systems eingeht (Mil02], vgl. Abschnitt 4.3), haben WeICKERT et al. Wei10] kürzlich eine allgemeinere Analyse eines quantenkritischen Endpunkts (QKEP) vorgenommen, wobei sich viele Eigenschaften aus nur drei Grundannahmen ableiten lassen:

1. Der kritische Anteil der freien Energie hat die Form $F_{\mathrm{kr}}=f(h, T, r)$, wobei $h=$ $H-H_{\mathrm{c}}$ den Abstand zum metamagnetischen kritischen Feld bezeichnet.

2. Am QKEP divergiert die magnetische Suszeptibilität, $\chi \rightarrow \infty$ für $T,|h| \rightarrow 0$.

3. Ein externer hydrostatischer Druck geht nur über die Druckabhängigkeit des metamagnetischen kritischen Feldes ein, $H_{\mathrm{c}}=H_{\mathrm{c}}(p)$.

Aus diesem phänomenologischen Ansatz folgen nur mit Hilfe von thermodynamischen Relationen ein Vorzeichenwechsel von $\alpha$ bei $H_{\mathrm{c}}, \alpha(h)=-\alpha(-h)$, sowie die Ähnlichkeit von Suszeptibilität und Magnetostriktion, $\chi \propto \lambda$. Das Verhalten von $\mathrm{Sr}_{3} \mathrm{Ru}_{2} \mathrm{O}_{7}$ in 
der Nähe des metamagnetischen Übergangs wird dadurch also schon gut beschrieben (vgl. Abschnitt 4.3). Sehr ähnliche experimentelle Ergebnisse sind schon seit längerem aus dem Schwere-Fermionen-System $\mathrm{CeRu}_{2} \mathrm{Si}_{2}$ bekannt und werden durch den phänomenologischen Ansatz ebenfalls gut beschrieben [Wei10. Ähnlich wie $\mathrm{Sr}_{3} \mathrm{Ru}_{2} \mathrm{O}_{7}$ zeigt $\mathrm{CeRu}_{2} \mathrm{Si}_{2}$ einen metamagnetischen Übergang ${ }^{5}$ bei $H_{\mathrm{c}} \approx 7,8 \mathrm{~T}$, unterscheidet sich aber ansonsten in fundamentalen mikroskopischen Eigenschaften [Flo02]: So sind die Elektronenmassen mit $m_{\mathrm{eff}} \approx 120 m_{e}$ deutlich größer und die Topologie der Fermifläche ist dreidimensional, während $\mathrm{Sr}_{3} \mathrm{Ru}_{2} \mathrm{O}_{7}$ noch kein Schwere-Fermionen-System darstellt und durch die Schichtstruktur einen äußerst zweidimensionalen elektronischen Charakter aufweist.

Als weitere Vorhersage läßt sich eine Proportionalität zwischen Suszeptibilität und Kompressibilität $\kappa$ bzw. Elastizitätsmodul $\mathcal{E}=1 / \kappa$ herleiten, bei tiefen Temperaturen also

$$
\kappa=-\frac{1}{V}\left(\frac{\partial V}{\partial p}\right)_{T} \propto \chi \rightarrow \infty \quad \text { für } H \rightarrow H_{\mathrm{c}} .
$$

Es ist bekannt, daß der Fermidruck des Elektronengases zwar nicht der einzige, aber doch ein wesentlicher Beitrag zur Kompressibilität von Metallen ist. Für einfache Alkalimetalle läßt sich der Elastizitätsmodul fast quantitativ, aber auch für Übergangsmetalle noch der Größenordnung nach mit dem Druck von freien Elektronen erklären AM76. Eine Divergenz der elektronischen Kompressibilität führt daher zwar nicht zu einer Instabilität des Kristalls, läßt aber in jedem Fall einen deutlichen Effekt auf die elastischen Eigenschaften erwarten. Eine „Aufweichung“ des Gitters ist also möglicherweise ein universelles Phänomen in der Nähe eines QKEPs. ${ }^{6}$

In der Tat beobachtet man in Ultraschallmessungen an $\mathrm{CeRu}_{2} \mathrm{Si}_{2}$ in der Nähe des kritischen Feldes eine Abnahme der longitudinalen Schallgeschwindigkeit um 30 Prozent Pue88. Da $c=\sqrt{\mathcal{E} / \varrho}$, bedeutet dies eine Halbierung des Elastizitätsmoduls, was als „Quasikollaps“ des Gitters bezeichnet wurde [Flo95].

Eine starke Kopplung an die elastischen Eigenschaften wurde kürzlich auch für den Fall einer Pomeranchuck-Instabilität mit $d_{x^{2}-y^{2}}$-Symmetrie, wie sie für $\mathrm{Sr}_{3} \mathrm{Ru}_{2} \mathrm{O}_{7}$ postuliert wurde, vorhergesagt AS09]. Ultraschalluntersuchungen an $\mathrm{Sr}_{3} \mathrm{Ru}_{2} \mathrm{O}_{7}$ wären damit eine interessante Möglichkeit, die nematische Phase näher zu charakterisieren. Solche Experimente liegen z. Zt. allerdings noch nicht vor.

Wird nun die Bildung der nematischen Phase durch eine Divergenz der Kompressibilität $\kappa$ begünstigt, so existiert möglicherweise ein kritischer Wert $\kappa_{\mathrm{c}}$, bei dessen Überschreitung das System gegen die nematische Ordnung instabil wird. Der Umriß der nematischen Phase wäre dann durch eine Linie konstanter Magnetostriktion mit $\lambda=$ const. $\cdot \kappa_{\mathrm{c}}$ gegeben. Wie die Auftragung in Abbildung 5.13 zeigt, kann diese

\footnotetext{
${ }^{5}$ Dieser bleibt bis zu tiefsten Temperaturen ein Crossover, stellt also keinen echten Phasenübergang dar. Ein QKEP ist damit weder in $\mathrm{Sr}_{3} \mathrm{Ru}_{2} \mathrm{O}_{7}$ noch in $\mathrm{CeRu}_{2} \mathrm{Si}_{2}$ exakt realisiert: Im ersten Fall wird er durch Bildung der nematischen Phase überdeckt, im letzteren ist der metamagnetische Übergang gewissermaßen „weiter" als bis zu $T=0$ unterdrückt Wei10.

${ }^{6}$ Diese Vorhersage ist eine Besonderheit für den Fall des quantenkritischen Endpunktes, der dadurch gegenüber einem gewöhnlichen QKP ausgezeichnet wird.
} 


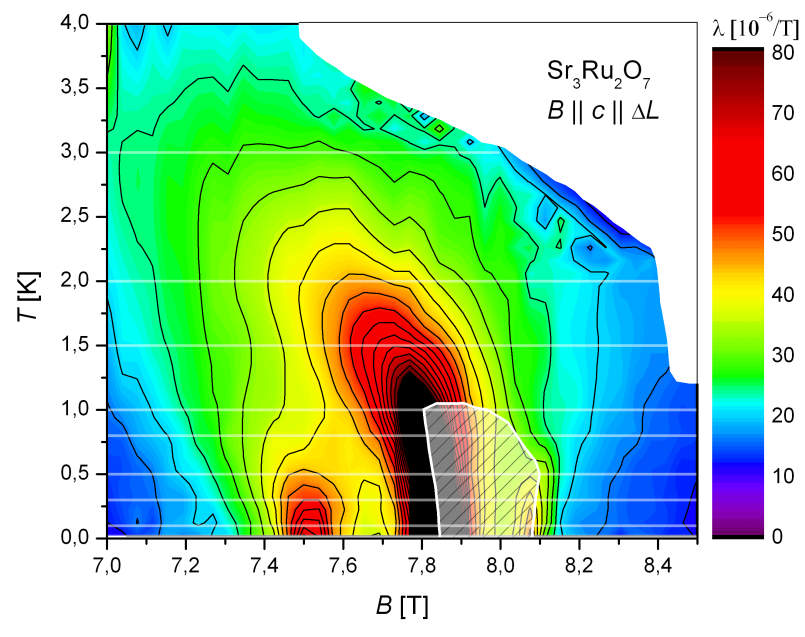

Abbildung 5.13: Konturlinien des Magnetostriktionskoeffizienten $\lambda$ für $B\|c\| \Delta L$ als Maß für die Stärke der quantenkritischen Fluktuationen. Der schraffierte Bereich entspricht der Lage der nematischen Phase; die horizontalen Linien zeigen die Lage der der Darstellung zugrundeliegenden Magnetostriktionskurven aus Geg06a.

einfache Annahme jedoch nicht unmittelbar bestätigt werden. Insbesondere die obere Phasengrenzlinie verläuft nicht parallel zu Linien konstanter Magnetostriktion, sondern schneidet sie vielmehr. In größerer Entfernung vom metamagnetischen Übergang weisen die Konturlinien zwar eine Form auf, die derjenigen der nematischen Phase stark ähnelt, wobei insbesondere die nach außen geneigten Flanken auffällig sind. Wie allerdings das Phasendiagramm in Abbildung 5.6 zeigt, sind die seitlichen Grenzen der Phase durch Orte nicht konstanter, sondern vielmehr maximaler Magnetostriktion gegeben. Die obigen Überlegungen zeigen damit, daß quantenkritische Fluktuationen durchaus eine wichtige Rolle für die nematische Ordnung spielen könnten, sich aber nicht in der konkreten Form der Phase niederschlagen.

\subsubsection{Nematischer Phasenübergang}

Ein weiteres wesentliches Ergebnis dieser Arbeit ist die Beobachtung der Phasengrenze am „Dach" in der thermischen Ausdehnung: für $B \| c$ zeigt sich dabei ein Sprung in $\alpha$, der von einer Spitze überlagert wird. Dies kann als Signatur eines klassischen Phasenübergangs zweiter Ordnung interpretiert werden. Mit zunehmender Verkippung des Magnetfelds wird die Spitze kleiner und es bleibt ein verbreiterter Sprung. Mögliche Interpretationen für diese Signatur wären ein mean-field-artiger Übergang zweiter Ordnung oder aber ein Crossover.

Kritische Analyse Äußerst aufschlußreich wäre nun eine Bestimmung des kritischen Exponenten am Übergang in die nematische Phase. Wäre dieser mit hinreichender Genauigkeit bekannt, so könnten Vergleiche mit anderen Festkörper- oder Modellsystemen vorgenommen oder der Phasenübergang womöglich einer Universalitätsklasse zugeordnet werden. Theoretische Berechnungen für den kritischen Exponenten der thermischen Ausdehnung sind oft nicht bekannt, allerdings gilt im Falle eines klassischen Phasenübergangs das Grüneisengesetz 2.13 mit $E_{0}=$ const. Spezifische Wärme 

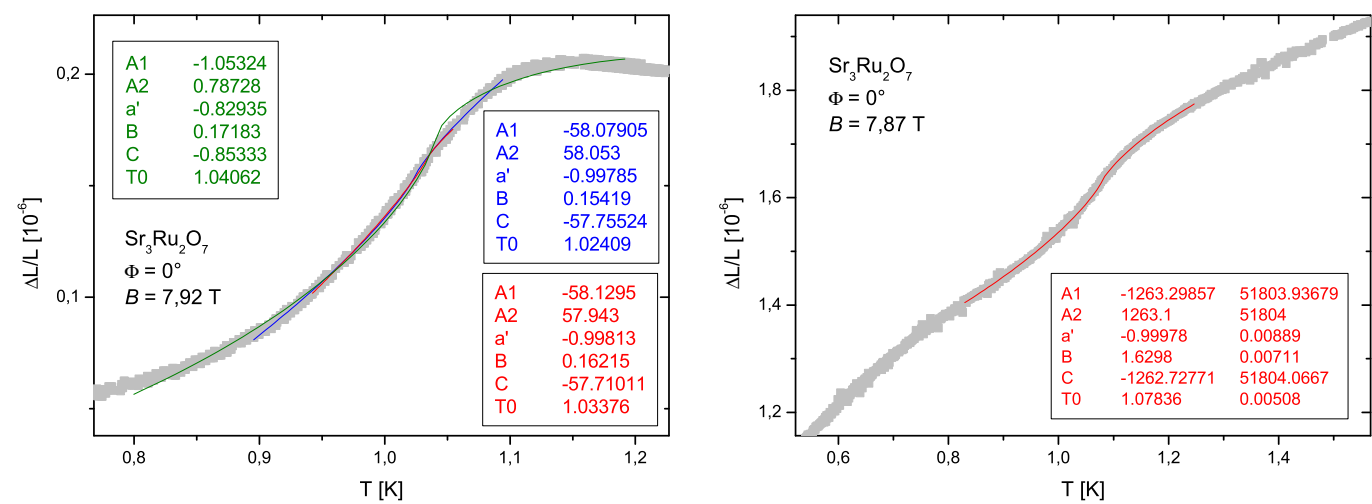

Abbildung 5.14: Versuch zur Bestimmung des kritischen Exponenten der thermischen Ausdehnung beim Eintritt in die nematische Phase durch Anpassen eines Potenzgesetzes. Links: der deutlichste Längensprung bei 7,92 T ist nicht sauber vom Hintergrund zu trennen (vgl. Text). Rechts: Bei 7,87 T ist der Sprung etwas kleiner, der Hintergrund hat allerdings eine günstigere Form.

und thermische Ausdehnung sind also einander proportional, siehe Gleichung (2.8). Für den kritischen Exponenten der thermischen Ausdehnung erwartet man deshalb den gleichen Wert wie für den der spezifischen Wärme.

Die Bestimmung von kritischen Exponenten ist nicht trivial und erfordert eine äußerst präzise Messung in unmittelbarer Nähe des Phasenübergangs [Wos07]. Für die kritische Analyse wurde deshalb der Bereich zwischen $0,6 \mathrm{~K}$ und $1,5 \mathrm{~K}$ bei $B=7,92 \mathrm{~T}$ (bei diesem Magnetfeld ist der Längensprung besonders ausgeprägt) äußerst langsam mit einer Rate von $180 \mathrm{mK} / \mathrm{h}$ vermessen und die Daten zusätzlich gemittelt.

Krellner et al. Kre09 konnten im System $\mathrm{YbRh}_{2} \mathrm{Si}_{2}$, welches beim antiferromagnetischen Phasenübergang ebenfalls einen Längensprung aufweist, den kritischen Exponenten der thermischen Ausdehnung direkt aus den Längendaten durch Anpassung der Funktion

$$
\frac{\Delta L}{L}(T)=\left(A_{1} \Theta(-t)+A_{2} \Theta(t)\right) \cdot|t|^{-a^{\prime}}+B+C t
$$

gewinnen, wobei $t=\left(T-T_{\mathrm{c}}\right) / T_{\mathrm{c}}$ die reduzierte Temperatur und $\Theta$ die Heavisidesche Sprungfunktion bezeichnet. Diese Form bietet eine Reihe von Vorteilen: die Bereiche $t<0$ und $t>0$ können in einer einzigen Anpassung behandelt werden, $T_{\mathrm{c}}$ ist ein freier Parameter und der Ableitungsschritt fällt weg. Da $\alpha=\mathrm{d} L(T) / \mathrm{d} T$, erhält man den kritischen Exponenten $a$ der thermischen Ausdehnung als $a=a^{\prime}+1$.

Abbildung 5.14 (links) zeigt eine Anpassung von Gleichung (5.8) an die Längendaten, die für verschiedene Temperaturbereiche durchgeführt wurde. Es zeigt sich ein dabei ein offensichtlicher Widerspruch: Der kritische Bereich von Phasenübergängen ist i. A. klein, $t \leq 10^{-1}$. Bei $T_{\mathrm{c}} \approx 1 \mathrm{~K}$ sind belastbare Ergebnisse also höchstens für einen Bereich von $\pm 100 \mathrm{mK}$ um den Phasenübergang zu erwarten. Bereits der Längensprung selbst nimmt jedoch etwa die gleiche Breite ein. Um ihn auf dem Hinter- 
grund zu detektieren, muß der Bereich für die Kurvenanpassung daher noch größer gewählt werden. Die grüne Kurve in Abbildung 5.8 entspricht einer Kurvenanpassung für $0,8 \mathrm{~K} \leq T \leq 1,2 \mathrm{~K}$ und liefert einen kritischen Exponenten von $a=0,173$. Allerdings kann sie, besonders im Randbereich, die Meßdaten nicht korrekt beschreiben. Problematisch ist hier besonders der Vorzeichenwechsel von positiver zu negativer Steigung, den $\Delta L / L$ von $T<T_{\mathrm{c}}$ zu $T>T_{\mathrm{c}}$ durchläuft und der durch den linearen Hintergrund nicht wiedergegeben werden kann. Die Wahl von kleineren Anpassungsbereichen (blaue und rote Kurven) erfaßt nicht den ganzen Sprung und liefert $a \approx 0$, womit keine Singularität in $\alpha$ mehr beschrieben wird.

Eine mögliche Abhilfe bestünde darin, den Hintergrund durch Terme höherer als linearer Ordnung zu beschreiben. Gleichung (5.8) hat aber einschließlich $T_{\mathrm{c}}$ bereits 6 freie Parameter und es zeigt sich, daß mit noch mehr Freiheitsgraden keine verwertbaren Ergebnisse für $a$ mehr erhalten werden können. Der theoretische Verlauf des Hintergrunds ist kompliziert, da er sich einerseits aus dem quantenkritischen Hintergrund und andererseits aus der thermischen Ausdehnung der nematischen Phase zusammensetzt. Er kann deshalb auch nicht ohne weiteres abgezogen werden, um den reinen Längensprung zu erhalten.

Abbildung 5.14 (rechts) zeigt einen weiteren Versuch für ein kleineres Feld von $B=7,87 \mathrm{~T}$. Der Längensprung ist hier weniger stark ausgeprägt, allerdings ist der Hintergrund annähernd linear. Eine Anpassung von Gleichung (5.8) über einen Bereich mit $|t| \leq 0,1$ ist hier besser möglich und beschreibt die Kurve zufriedenstellend. Für den kritischen Exponenten ergibt sich ein außerordentlich kleiner Wert $a=0,0002$, der vom Fehler $(0,0089)$ weit überstiegen wird. Ein Wert $a=0$ entspricht einerseits dem Ergebnis eines Mean-Field-Szenarios, in welchem keine Fluktuationen berücksichtigt sind und für $\alpha$ ein Sprung erwartet wird. Da $\alpha$ aber im unverkippten Feld keinen reinen Sprung, sondern vielmehr eine deutliche Spitze aufweist, liegt Mean-Field-Verhalten offensichtlich nicht vor. Eine zweite Möglichkeit wäre das Vorliegen einer logarithmischen Divergenz, die allerdings durch die Funktion (5.8) nicht beschrieben werden kann. Es wird deshalb ein weiterer Anpassungsversuch mit einer neuen Funktion unternommen. Aus $\alpha \propto \log |t|^{-a}$ folgt durch Integration:

$$
\frac{\Delta L}{L}(T)=\left(A_{1} \Theta(-t)+A_{2} \Theta(t)\right) \cdot(-a) \cdot(|t| \log |t|-|t|)+B+C t
$$

Der Exponent $a$ spielt im Falle der logarithmischen Divergenz keine Rolle und kann in die Konstanten $A_{1}$ und $A_{2}$ absorbiert werden.

Abbildung 5.15 zeigt die Anpassung von Gleichung (5.9) an die Längendaten bei 7,87 T. Die Daten lassen sich grundsätzlich durch die angepaßte Funktion beschreiben, sind also konsistent mit einer logarithmischen Divergenz von $\alpha$ am kritischen Punkt. Wie die ähnlich gute Anpassung in Abbildung 5.14 zeigt, ist das Verhalten aber nicht von einem Potenzgesetz mit einem kleinen Exponenten $a \leq 0,1 \mathrm{zu}$ unterscheiden.

Universalitätsklasse Unabhängig von der experimentellen Bestimmung eines kritischen Exponenten läßt sich die Natur des Phasenübergangs unter den im vorigen 


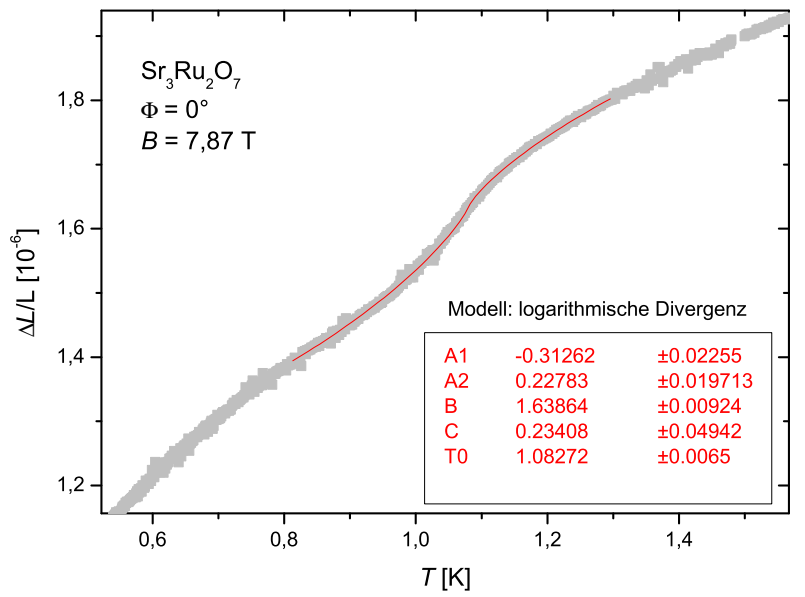

Abbildung 5.15: Der Längensprung beim Eintritt in die nematische Phase kann unter Annahme einer logarithmischen Divergenz von $\alpha$ angepaßt werden.

Abschnitt über das Modell der partiellen orbitalen Ordnung gemachten Annahmen diskutieren. Demnach kommt es unterhalb von $T_{\mathrm{c}}$ zu einer spontanen Brechung der vierzähligen Rotationssymmetrie des Kristallgitters in der Ebene, wobei im unverkippten Feld die zwei als $N^{+}$und $N^{-}$bezeichneten energetisch äquivalenten Zustände eingenommen werden können. Der zugehörige Ordnungsparameter ist ein Skalar und hat die Dimension $n=1$. Die nematische Ordnung spielt sich in der Ebene ab, so daß die effektive Dimensionalität des Systems $d=2$ ist. Damit ist gerade die Universalitätsklasse des zweidimensionalen Isingmodells beschrieben. Die Äquivalenz zwischen der nematischen Ordnung und einem Isingmodell läßt sich veranschaulichen, wenn man z. B. den $N^{+}$- als Spin-Up( $\left.\uparrow\right)$ - und den $N^{-}$- als Spin-Down $(\downarrow)$-Zustand identifiziert. Wird das Magnetfeld in die Ebene gekippt, so wird eine der zwei Varianten $N^{+}, \mathrm{N}^{-}$ energetisch bevorzugt. Die In-Plane-Komponente des Magnetfelds ist damit äquivalent zum Anlegen eines externen Magnetfelds an einen Ising-Magneten. Sowohl im Modell der orbitalen Ordnung als auch im Isingsystem liegt damit ein Fall von expliziter Symmetriebrechung vor. Im Folgenden soll nun diskutiert werden, ob die experimentellen Daten diese einfachen Überlegungen rechtfertigen.

Zum Verhalten des zweidimensionalen Isingmodells in externen Magnetfeldern hat A. KALz Monte-Carlo-Simulationen auf Gittern mit einer Kantenlänge von $L=50-$ 100 Gitterplätzen ${ }^{7}$ durchgeführt Kal11. Abbildung 5.16 (links) zeigt, daß die spezifische Wärme $c$ als Funktion der Temperatur im Nullfeld die bekannte logarithmische Divergenz aufweist (vgl. Tabelle 2.1). Werden nun nach und nach stärkere externe Felder angelegt, so wird das Maximum in $c$ kleiner, breiter. und verschiebt sich zu höheren Temperaturen. Durch stärkere Magnetfelder ist die Symmetrie zwischen den zwei möglichen Spinzuständen explizit gebrochen, so daß hier nicht mehr von einem Phasenübergang zweiter Ordnung, sondern nur noch von einem "Crossover" gesprochen werden kann. Da kritische Fluktuationen unterdrückt werden, verschwindet die

\footnotetext{
${ }^{7}$ Die Systemgröße ist so groß gewählt, daß sie keinen Einfluß mehr auf die ermittelten thermodynamischen Größen hat, diese als Funktion von $L$ also konvergiert sind.
} 

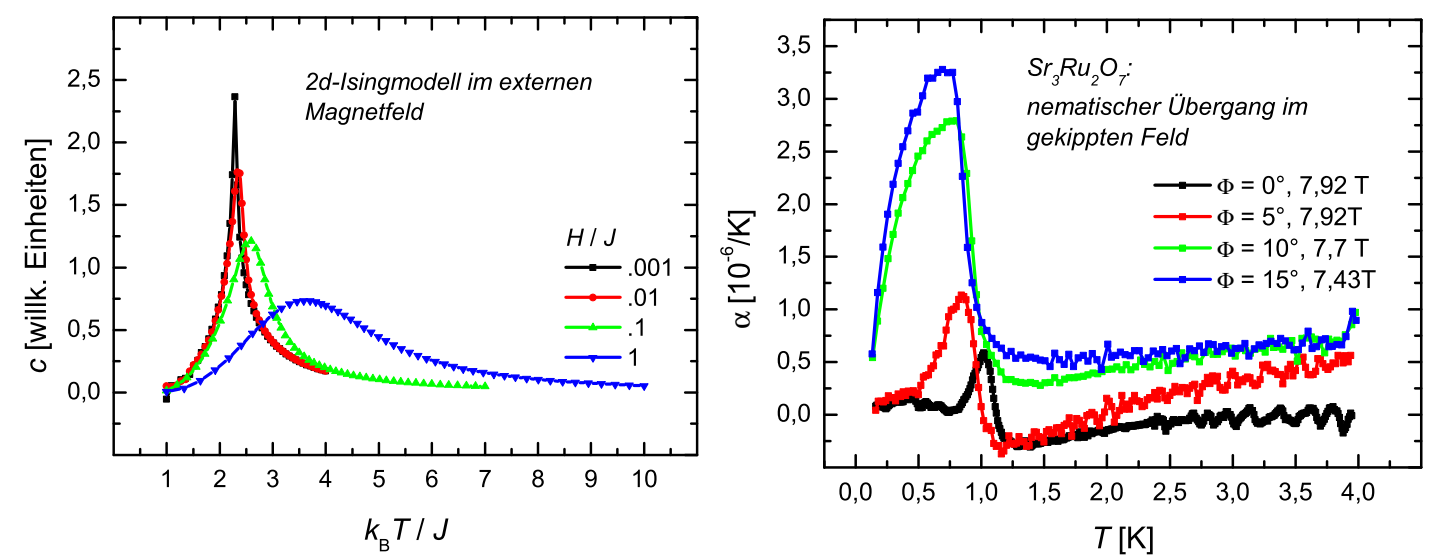

Abbildung 5.16: Phasenübergänge zweiter Ordnung unter dem Einfluß expliziter Symmetriebrechung. Links: spezifische Wärme des 2d-Isingmodells aus einer Simulation von A. Kalz Kal11]. Rechts: Thermische Ausdehnung in $\mathrm{Sr}_{3} \mathrm{Ru}_{2} \mathrm{O}_{7}$.

scharfe Spitze bei $T_{\mathrm{c}}$ im äußeren Feld.

Dieses Verhalten des Modellsystems kann nun mit der in Abbildung 5.16 (rechts) gezeigten Entwicklung des nematischen Phasenübergangs verglichen werden. Für $B \| c$ ist der Übergang durch einen Sprung in $\alpha$ gekennzeichnet. Im Gegensatz zum Modellsystem wächst dieser jedoch mit zunehmender Verkippung stark an. Eine nennenswerte Verbreiterung des Übergangs ist nicht festzustellen. Weiterhin entwickelt sich die Übergangstemperatur für steigende Verkippung zu kleineren statt zu größeren Werten. Die Spitze, die den Sprung in $\alpha$ für $B \| c$ überlagert, verschwindet durch Feldverkippung rasch.

Der letztgenannte Effekt läßt sich in Analogie zum Modellsystem verstehen: da der Eintritt in die verzerrte Phase im unverkippten Feld mit starken Fluktuationen zwischen den zwei äquivalenten Verzerrungsvarianten $N^{+}$und $N^{-}$einhergehen sollte, sind deutliche Fluktuationseffekte zu erwarten, die sich in der beobachteten Spitze in $\alpha$ äußern. Davon abgesehen zeigen aber deutliche Unterschiede zwischen Meßdaten und Simulation, daß die Annahme eines Ising-artigen nematischen Phasenübergangs zu kurz greift. So wird im Isingmodell die Wechselwirkung zwischen den Spins durch eine einzige, temperatur- und feldunabhängige Konstante $J$ beschrieben. Wie diese Wechselwirkung im Fall der nematischen Phase aussieht, ist dagegen nicht ohne weiteres klar. Da das kritische metamagnetische Feld $H_{\mathrm{mm}}$ aber stark winkelabhängig ist, gilt dasselbe möglicherweise auch für die Stärke der Wechselwirkung.

Weiterhin existieren im Isingmagneten die zwei möglichen Zustände $\uparrow$ und $\downarrow$ unabhängig von Temperatur oder Feld. Da leider keine Rechnungen zum nematischen Ordnungsparameter bei endlicher Temperatur existieren, kann diese Annahme nicht ohne weiteres auf das Modell der partiellen orbitalen Ordnung übertragen werden. Würde der Zusammenbruch der nematischen Ordnung für $T>1 \mathrm{~K}$ nur auf der thermischen Unordnung der Zustände $N^{+}$und $N^{-}$beruhen, so wäre wie beim Isingmodell 
eine Verbreiterung des Übergangs zu erwarten, die aber nicht beobachtet wird. Außerdem geht der Ordnungsparameter des Isingmodells für $T \rightarrow T_{\mathrm{c}}$ kontinuierlich und glatt gegen null. Betrachtet man dazu die Überlegungen des Abschnitts 5.2.1 zur Temperaturabhängigkeit und insbesondere Gleichung (5.5), so sollte sich auch $\Delta L(T)$ bei $T_{\mathrm{c}}$ ähnlich glatt verhalten, zumindest aber nicht die beobachtete sprunghafte Änderung zeigen.

Abschließend muß auch die anfangs gemachte Annahme eines gültigen Grüneisengesetzes kritisch hinterfragt werden. Während die nematische Phase eine Fermiflüssigkeit bildet, befindet sich das System oberhalb des Phasenübergangs im NFF-Bereich [Gri01, so daß eine Verletzung des Grüneisengesetzes nicht auszuschließen ist. Dann allerdings wären die Signaturen von thermischer Ausdehnung $\alpha$ und spezifischer Wärme $c$ schon prinzipiell nicht vergleichbar. In der Tat wurde in $c(T)$ beim Eintritt in die Phase im unverkippten Feld nur ein Sprung ohne die in $\alpha$ sichtbare überlagerte Spitze gefunden, vgl. Abschnitt 4.5. Der gleiche Übergang von einem NFF-Zustand in eine geordnete FF-Phase liegt allerdings auch im oben erwähnten Fall von $\mathrm{YbRh}_{2} \mathrm{Si}_{2}$ vor, wo das Grüneisengesetz offensichtlich erfüllt ist Kre09. ${ }^{8}$

Für die Äquivalenz der Modelle spricht, daß die Signatur von $\alpha$ am Phasenübergang konsistent mit einer logarithmischen Divergenz ist, wie sie für ein 2d-Ising-System erwartet wird. Auf Grundlage der Meßdaten können andere Exponenten aber nicht ausgeschlossen werden. Auch angesichts der oben diskutierten Unterschiede kann die Analyse deshalb die Natur des kritischen Verhaltens nicht vollständig klären.

\subsection{Entropie}

Nachdem in dieser Arbeit die thermische Ausdehnung von $\mathrm{Sr}_{3} \mathrm{Ru}_{2} \mathrm{O}_{7}$ in der $a b$-Ebene bestimmt wurde, läßt sich zusammen mit den Daten, die GEgEnwarT et al. Geg06b. schon früher für die $c$-Richtung bestimmt haben, der Volumenausdehnungskoeffizient $\beta$ berechnen. Da es sich um ein tetragonales System handelt, gilt $\beta=\alpha_{c}+2 \cdot \alpha_{a b}$. Für die Analyse wird sowohl von $\alpha_{c}$ als auch von $\alpha_{a b}$ der in Abschnitt 5.1.1 gefundene isotrope FF-Hintergrund abgezogen. Bei einigen Kurven, die nicht genau beim gleichen Feldwert vorliegen, wurde zwischen benachbarten Magnetfeldern linear interpoliert. Die so erhaltenen Kurven $\beta(T)$ zeigen qualitativ das gleiche quantenkritische Verhalten wie $\alpha_{c}$, enthalten allerdings zusätzlich den aus dem Beitrag von $\alpha_{a b}$ stammenden Längensprung als Signatur des nematischen Übergangs.

Ist $\beta$ als Funktion von $H$ bekannt, so läßt sich außerdem unter gewissen Voraussetzungen die Entropie $S(H)$ bestimmen. Durch eine solche Auswertung können einerseits andere bekannte Messungen der Entropie überprüft werden, weiterhin aber auch die unterschiedlichen thermodynamischen Größen auf ihre Konsistenz getestet werden.

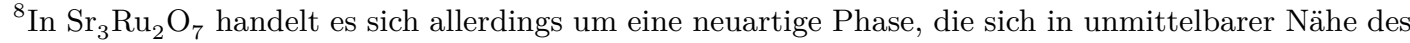
QKPs bildet, während im Fall von $\mathrm{YbRh}_{2} \mathrm{Si}_{2}$ klassische antiferromagnetische Ordnung vorliegt, aus deren Unterdrückung der QKP erst hervorgeht. Im Phasendiagramm erfolgt die Bestimmung der kritischen Exponenten deshalb in $\mathrm{YbRh}_{2} \mathrm{Si}_{2}$ abseits des QKP und nicht unmittelbar über ihm.
} 

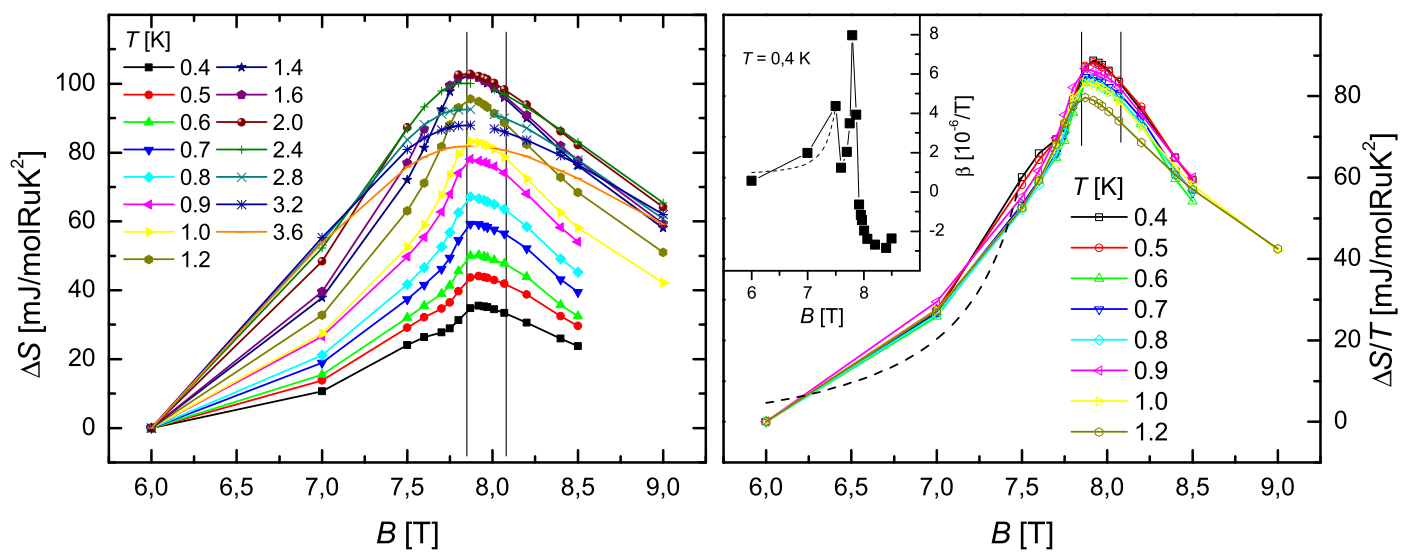

Abbildung 5.17: Aus dem Volumenausdehnungskoeffizienten nach G1. 5.11 berechnete Entropie von $\mathrm{Sr}_{3} \mathrm{Ru}_{2} \mathrm{O}_{7}$. Links: Auf beiden Seiten der nematischen Phase (die senkrechten Linien markieren ihre Lage) steigt die Entropie stark an, dieser Anstieg wird in der Phase abgeschnitten. Rechts: Im Fermiflüssigkeitsbereich, insb. auch innerhalb der nematischen Phase, ist $S / T$ temperaturunabhängig.

Unter der auf Seite 68 vorgestellten Annahme, daß der Druck in die freie Energie nur in der Form eines druckabhängigen kritischen Felds eingeht, d. h. $f$ von der Form $f=f\left(T, H-H_{\mathrm{c}}(p)\right)$ ist, ergibt sich die Entropie wie folgt aus dem Volumenausdehnungskoeffizienten $\beta$ :

$$
\begin{gathered}
\beta=-\frac{1}{V} \frac{\partial S}{\partial p}=-\frac{1}{V} \frac{\partial S}{\partial H_{\mathrm{c}}} \frac{\partial H_{\mathrm{c}}}{\partial p} \\
=\frac{1}{V} \frac{\partial S}{\partial H} \frac{\partial H_{\mathrm{c}}}{\partial p} \\
\Rightarrow S(H)-S\left(H_{0}\right)=V\left(\frac{\partial H_{\mathrm{c}}}{\partial p}\right)^{-1} \int_{H_{0}}^{H} \beta\left(H^{\prime}\right) \mathrm{d} H^{\prime}
\end{gathered}
$$

Für $\mu_{0} \mathrm{~d} H_{\mathrm{c}} / \mathrm{d} p$ wird der bereits oben verwendete Wert von 5,6 T/GPa Chi02, Geg06a angenommen. Als untere Integrationsgrenze wird ein Feld von $6 \mathrm{~T}$ gewählt. Das Molvolumen pro Ru-Atom ergibt sich aus dem Produkt der Gitterkonstanten $a, b$ und $c$ mit den in Abschnitt 4.1 angegebenen Werten, wobei die pseudotetragonale Einheitszelle $8 \mathrm{Ru}$-Atome enthält (sie ist doppelt so groß wie die tetragonale Einheitszelle, welche $4 \mathrm{Ru}$-Atome einschließt, vgl. Abbildung 4.1 auf Seite 38). Damit errechnet man $V_{\mathrm{mol}}^{\mathrm{Ru}}=1 / 8 \cdot N_{\mathrm{A}} \cdot a \cdot b \cdot c=47,2 \mathrm{~cm}^{3}$.

In Abbildung 5.17 (links) sind die durch Integration nach der Trapezregel berechneten Entropien für verschiedene Temperaturen aufgetragen. Auf beiden Seiten der nematischen Phase steigt $S$ mit Annäherung an das kritische Feld. Es kommt aber nicht zu einer Divergenz, da der Anstieg im Bereich der Phase abgeschnitten wird. Bei Temperaturen oberhalb von $1,2 \mathrm{~K}$ ist eine Abflachung nicht mehr zu beobachten 

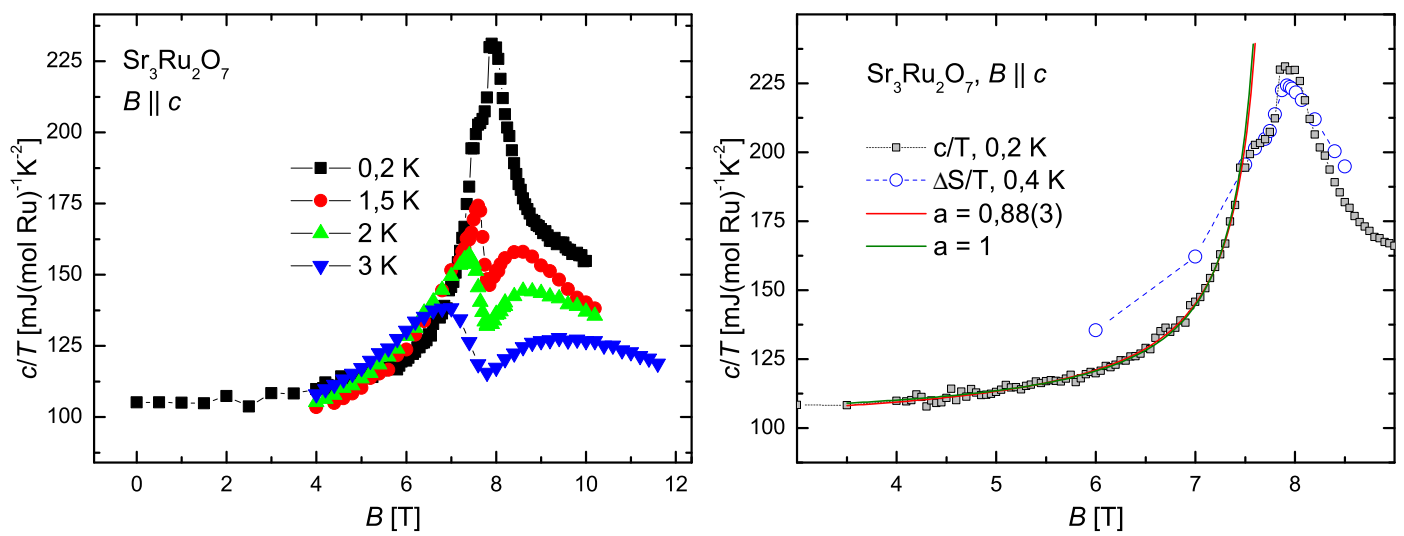

Abbildung 5.18: Spezifische Wärme von $\mathrm{Sr}_{3} \mathrm{Ru}_{2} \mathrm{O}_{7}$ für $B \| c$ nach Daten von Y. TokiwA. Links: Für höhere Temperaturen beobachtet man eine charakteristische Doppelspitze beim kritischen Feld. Rechts: Die aus der thermischen Ausdehnung $\beta$ berechnte Entropie zeigt eine gute Übereinstimmung.

und $S$ zeigt nur noch ein breites Maximum im Bereich des kritischen Feldes. In die Auftragung von $S / T$ für kleinere Temperaturen in Abbildung 5.17 (rechts) fallen die Daten für unterschiedliche Temperaturen mit guter Genauigkeit zusammen, wie für eine Fermiflüssigkeit zu erwarten ist.

Bei $B=7,5 \mathrm{~T}$ liest man für $\Delta S / T$ ca. $50 \mathrm{~mJ} / \mathrm{molRuK}^{2}$ ab. Rost et al. Ros09 haben aus Messungen des magnetokalorischen Effekts ebenfalls einen Wert von $S(B=$ $7,5 \mathrm{~T})-S(B=6 \mathrm{~T}) \approx 50 \mathrm{~mJ} / \mathrm{molRuK}^{2}$ erhalten, s. hierzu Abbildung 4.10 . Es zeigt sich also eine sehr gute quantitative Übereinstimmung der aus der Messung unterschiedlicher physikalischer Effekte bestimmten Entropien. Dies zeigt zum einen, daß die Messungen untereinander thermodynamisch konsistent sind. Weiterhin demonstriert die Übereinstimmung, daß der Wert für die Druckabhängigkeit des kritischen Feldes aus Chi02 korrekt ist. Schließlich wird die Gültigkeit der in 5.10 gemachten Annahmen bestätigt, was das allgemeine Modell von WEICKERT et al. [Wei10] untermauert, wonach die Thermodynamik des Systems ganz wesentlich durch den Abstand vom kritischen Magnetfeld bestimmt wird.

\subsection{Spezifische Wärme}

Ergänzend zu den aus der Dilatometrie gewonnenen Ergebnissen sollen zusätzlich einige Messungen der spezifischen Wärme betrachtet werden, die von Y. TokiwA an der gleichen Probe (C660a) mittels einer semi-adiabatischen Heizpulsmethode vorgenommen wurden. In Abbildung 5.18 ist der Sommerfeldkoeffizienten $\gamma=c / T$ in der Nähe des metamagnetischen kritischen Feldes $H_{\mathrm{c}}^{\mathrm{mm}}$ für unterschiedliche Temperaturen aufgetragen. Der Wert im Nullfeld beträgt bei $0,25 \mathrm{~K}$ etwa $110 \mathrm{~mJ} / \mathrm{molRuK}^{2}$, was konsistent mit anderen veröffentlichten Daten ist [Ros10]. 
Für $T \geq 1,5 \mathrm{~K}$ zeigt sich eine charakteristische Struktur aus zwei Maxima beiderseits des kritischen Feldes, welche ein Minimum bei $H_{\mathrm{c}}^{\mathrm{mm}}$ zwischen sich einschließen. Für tiefe Temperaturen entwickelt sich die Struktur dagegen zu einem einzigen Maximum bei $H_{\mathrm{c}}^{\mathrm{mm}}$.

Die Beobachtungen lassen sich wiederum im bereits in Abschnitt 5.2 .2 behandelten allgemeinen Szenario metamagnetischer Quantenkritikalität diskutieren. Die magnetische Suszeptibilität, welche in diesem Szenario als Funktion von $T$ divergiert,

$$
\chi\left(T, H_{\mathrm{c}}^{\mathrm{mm}}\right) \rightarrow \infty \text { für } T \rightarrow 0
$$

ist über die Maxwellrelation

$$
\frac{\partial^{2} \gamma}{\partial H^{2}}=\frac{\partial^{2} \chi}{\partial T^{2}}
$$

mit dem Koeffizienten $\gamma$ der spezifischen Wärme verknüpft [Wei10]. Aus der Divergenz von $\chi\left(T, H_{\mathrm{c}}^{\mathrm{mm}}\right)$, welche eine positive Krümmung bedeutet, folgt damit ein Minimum von $\gamma(H)$ bei $H_{\mathrm{c}}^{\mathrm{mm}}$. Da $\gamma(H)$ zu beiden Seiten von $H_{\mathrm{c}}^{\mathrm{mm}}$ nicht beliebig weit ansteigen kann, enstehen notwendigerweise zwei Maxima.

Die Divergenz von $\chi$ setzt sich zu tiefen Temperaturen hin nicht beliebig weit fort, sondern wird im Bereich der nematischen Phase abgeschnitten, so daß $\chi$ sättigt Gri03. Damit ändert sich auch das Vorzeichen der Krümmung, so daß bei tiefen Temperaturen ein Maximum von $\gamma(H)$ bei $H_{\mathrm{c}}^{\mathrm{mm}}$ ensteht.

Wiederum kann ein Vergleich zum System $\mathrm{CeRu}_{2} \mathrm{Si}_{2}$ gezogen werden, in welchem schon früher die oben beschriebene „Doppelspitze“ in der spezifischen Wärme gefunden wurde Aok98. Die gleiche Struktur wurde auch für $\mathrm{Sr}_{3} \mathrm{Ru}_{2} \mathrm{O}_{7}$ vorhergesagt Wei10] und ist nun mit den Daten in Abbildung 5.18 bestätigt. Damit gibt es einen weiteren Anhaltspunkt für den Einfluß metamagnetischer Quantenfluktuationen in $\mathrm{Sr}_{3} \mathrm{Ru}_{2} \mathrm{O}_{7}$.

Interessant wäre noch eine Bestimmung der analytischen Abhängigkeit $\Delta S(H-$ $\left.H_{\mathrm{c}}\right)$ und ein Vergleich mit der ebenfalls in Ros09 gefundenen Divergenz $\Delta S(H-$ $\left.H_{\mathrm{c}}\right) \propto\left(H-H_{\mathrm{c}}\right)^{-1}$. Dies stellte eine Abweichung von den Vorhersagen der HM-Theorie dar, vgl. Seite 47. Die Kurven in Abbildung 5.18 (rechts) zeigen die Anpassung einer Divergenz $\Delta c \propto h^{-a}$, wobei in $h=H-H_{\mathrm{c}}$ das kritische Feld mit $H_{\mathrm{c}}=7,85 \mathrm{~T}$ festgesetzt ist. Eine Anpassung mit $a$ als freiem Parameter liefert $a=0,88(3)$, also eine weniger steile Divergenz als in [Ros09]. Dies stellt zumindest der Tendenz nach eine Annäherung an das HM-Szenario dar, ist aber immer noch vom vorhergesagten Wert $a=1 / 3$ entfernt. Mit einem festen Exponenten $a=1$ ergibt sich allerdings eine fast ebenso gute Beschreibung der Daten, die somit nicht im groben Widerspruch zum in $[$ Ros09 gefundenen Exponenten stehen. Es gilt allerdings nach wie vor die auf Seite 47 diskutierte Einschränkung, daß die untersuchten Daten einen relativ großen Hintergrundsbeitrag haben, der möglicherweise selbst feldabhängig ist. Der erhaltene Exponent gibt also nicht notwendigerweise allein das kritische Verhalten wieder.

Zusätzlich ist in Abbildung 5.18 (rechts) noch einmal die aus der thermischen Ausdehnung $\beta$ berechnte Entropie $\Delta S / T$, die nach Gleichung (4.4) bis auf eine Konstante der spezifischen Wärme entsprechen sollte. Auch wenn die quantitative Übereinstim- 
mung nicht perfekt ist, zeigt sich hier wiederum die gute Konsistenz der auf unterschiedliche Weise bestimmten thermodynamischen Größen. 



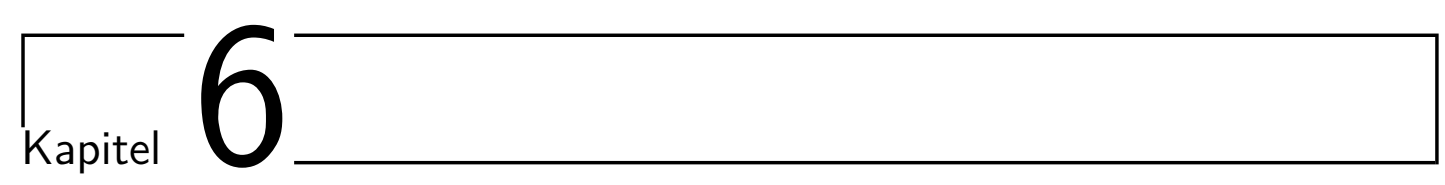

\section{Zusammenfassung}

Mit dem Material $\mathrm{Sr}_{3} \mathrm{Ru}_{2} \mathrm{O}_{7}$ hat sich die vorliegende Arbeit mit einem System befaßt, welches sich durch Phänomene auszeichnet, die in der aktuellen Festkörperforschung von großem Interesse sind. Die Unterdrückung eines metamagnetischen Übergangs, welcher durch eine Singularität in der elektronischen Zustandsdichte hervorgerufen wird, zum Temperaturnullpunkt führt zu einem quantenkritischen Endpunkt. Die damit verbundenen Quantenfluktuationen koppeln stark an das Gitter, und nahe des quantenkritischen Endpunkts bildet sich eine neuartige Phase. Während das Kristallgitter in der $a b$-Ebene eine vierzählige Rotationssymmetrie besitzt, wird diese vom Elektronensystem in dieser nematischen Phase spontan gebrochen.

Zur Charakterisierung dieser nematischen Phase wurden hochauflösende Messungen von thermischer Ausdehnung und Magnetostriktion bei tiefen Temperaturen und hohen Magnetfeldern durchgeführt. Ein miniaturisiertes Dilatometer, welches sich in verschiedenen Konfigurationen in einen ${ }^{3} \mathrm{He}-{ }^{4} \mathrm{He}-\mathrm{Mischungskryostaten}$ einbauen ließ, ermöglichte es, die Auswirkungen der nematischen Ordnung auf das Gitter in unterschiedlichen Richtungen zu studieren.

Das wesentliche experimentelle Ergebnis dieser Arbeit ist die Existenz einer symmetriebrechenden Gitterverzerrung innerhalb der nematischen Phase. Da die relative Längenänderung $\Delta L / L$ lediglich von der Größenordnung $10^{-6}$ und damit unterhalb der Auflösungsgrenze beispielsweise von Neutronenbeugungsexperimenten ist, konnte sie in früheren Messungen nicht detektiert werden. Durch einen Vergleich mit Widerstandsmessungen konnte gezeigt werden, daß es parallel zur leichten Transportachse zu einer Expansion des Gitters kommt, während es sich in der Richtung der schweren Achse zusammenzieht. Mit dieser Beziehung, die übereinstimmend aus thermischer Ausdehnung und Magnetostriktion hervorgeht, ist eine Randbedingung für alle Theorien der elektronischen Nematizität in $\mathrm{Sr}_{3} \mathrm{Ru}_{2} \mathrm{O}_{7}$ gegeben. Weiterhin zeichnet sich die nematische Phase durch eine gleichförmige thermische Ausdehnung der Form $\Delta L_{\|}+\Delta L_{\perp}=0$ aus. Dies ist besonders bemerkenswert, da außerhalb der Phase bereits die Magnetfeldkomponente in der Ebene eine anisotrope thermische Ausdehnung bewirkt. 
Wie auch in den Widerstandsexperimenten tritt die Symmetriebrechung, obwohl inhärent vorhanden, erst bei einer teilweisen Verkippung des Magnetfelds in die $a b$ Ebene in Erscheinung. An dieser Stelle sei noch einmal auf die Abbildung 5.8 auf Seite 60 verwiesen, in der bei einem Winkel von $5^{\circ}$ der Effekt besonders deutlich wird: Bei hohen Temperaturen ist die thermische Ausdehnung isotrop, während sich bei Abkühlung in die nematische Phase eine spontane Gitterverzerrung entwickelt.

Die gefundene Verzerrung wurde im Rahmen eines kürzlich veröffentlichten theoretischen Modells diskutiert, welches die nematische Phase als eine Form von partieller orbitaler Ordnung innerhalb einer komplexen Bandstruktur beschreibt. Dies führt zur Bildung einer Struktur von Domänen, welche in verkippten Feldern eine längliche Geometrie annehmen. Zusammen mit dem antibindenden Charakter der beteiligten quasieindimensionalen Bänder konnten damit die im spezifischen Widerstand und in den dilatometrischen Messungen gefundenen Anisotropien konsistent beschrieben werden.

Ein weiteres wesentliches Ergebnis dieser Arbeit ist die Beobachtung eines deutlichen Längensprungs, der beim Eintritt in die nematische Phase als Funktion der Temperatur auftritt. Während es in der $c$-Richtung nur schwache Anomalien in der thermischen Ausdehnung gibt, konnte damit in der $a b$-Ebene eine deutliche thermodynamische Signatur eines Phasenübergangs zweiter Ordnung gefunden werden. Im Rahmen des Modells der partiellen orbitalen Ordnung kann der Übergang in erster Näherung in die 2d-Ising-Universalitätsklasse eingeordnet werden und das kritische Verhalten am Phasenübergang konnte mit der für diese Klasse erwarteten logarithmischen Divergenz des thermischen Ausdehnungskoeffizienten angepaßt werden. Die Anpassung ist allerdings nicht eindeutig, und der Vergleich mit Simulationen ergibt besonders im Fall expliziter Symmetriebrechung deutliche Abweichungen. Es ist deshalb nicht sicher, ob der nematische Phasenübergang klassisch-universelles kritisches Verhalten zeigt.

Neben den im besprochenen Modell berücksichtigten Bandstruktureffekten gibt es, wie auf Seite 68 beschrieben, starke Hinweise auf quantenkritische Einflüsse. Die gefundene Verzerrung kann somit auch in den Zusammenhang allgemeiner Vorhersagen von strukturellen Instabilitäten in der Nähe von quantenkritischen Punkten eingeordnet werden. Inwiefern Quantenfluktuationen die Bildung der nematischen Phase hervorrufen, bleibt eine spannende Fragestellung.

Aus den Resultaten dieser Arbeit eröffnen sich weitere Arbeitsansätze sowohl auf experimenteller als auch theoretischer Seite.

Oben wurden die in spezifischem Widerstand und Ausdehnung gefundenen Anisotropien diskutiert. Bei der experimentellen Bestimmung dieser Größen schließen sich die Anforderungen an die Probengeometrie in gewisser Hinsicht gegenseitig aus: Für Transportmessungen ist ein großes Aspektverhältnis, d. h. eine lange Probe mit einem kleinen Querschnitt wünschenswert, denn nur so entsteht bei einem sehr kleinen spezifischen Widerstand ein hinreichend großes Signal. Das Dilatometer erfordert dagegen einen großen Querschnitt, um bei gegebener Federkraft den Druck auf die Probe klein zu halten. Gelänge es, einen Kompromiß zwischen diesen Anforderungen zu finden, so 
wäre eine simultane Messung der zwei Größen sicherlich aufschlußreich. Dies gilt besonders angesichts der auf Seite 65 angesprochenen Vermutung, daß uniaxialer Druck im unverkippten Feld eine der zwei Domänenvarianten bevorzugt. Um dies zu bestätigen, sollte untersucht werden, ob durch Anlegen eines uniaxialen Drucks Einfluß auf die Widerstandsanisotropie genommen werden kann.

Weiterhin wären genauere Messungen im Bereich des nematischen Phasenübergangs wichtig, um zu klären, inwieweit möglicherweise universelles Verhalten vorliegt. Der Einfluß einer expliziten Symmetriebrechung durch ein verkipptes Feld auf die Signatur des Übergangs sollte beispielsweise auch für die spezifische Wärme untersucht werden.

Die Arbeit zeigt allgemein, daß mit der Dilatometrie ein hochempfindliches Werkzeug zur Untersuchung von symmetriebrechenden Phänomenen wie der elektronischen nematischen Ordnung existiert. Es ist deshalb zu hoffen, daß die vorliegenden Ergebnisse auch von theoretischer Seite eine stärkere Berücksichtigung der Kopplung von elektronischen Freiheitsgraden an das Kristallgitter motivieren. In Bezug auf Modelle für $\mathrm{Sr}_{3} \mathrm{Ru}_{2} \mathrm{O}_{7}$ wäre es beispielsweise wünschenswert, nicht nur das Vorzeichen, sondern auch die Größenordnung der Verzerrung erklären zu können. Außerdem sind Rechnungen bei endlicher Temperatur notwendig, um über die Temperaturabhängigkeit des Ordnungsparameters die gleichförmige Verzerrung und auch die kontinuierliche Abnahme der anisotropen Streuung zu verstehen.

Der obige Ausblick zeigt, daß das untersuchte System sicherlich noch weitere interessante Problemstellungen und offene Fragen bereithält. Ganz allgemein kann abschließend festgehalten werden, daß es sich bei den Ergebnissen dieser Arbeit um die erste Beobachtung einer strukturellen Instabilität nahe eines quantenkritischen Punktes handelt. 



\section{Literatur}

[AM76] N. W. Ashcroft und D. N. Mermin: Solid State Physics. Thomson Learning (1976)

[AN71] J. Als-Nielsen, O. W. Dietrich, W. Kunnmann und L. Passell: Critical Behavior of the Heisenberg Ferromagnets EuO and EuS. Phys. Rev. Lett. 27, 741-744 (1971)

[AS09] H. Adachi und M. Sigrist: Probing the $d_{x^{2}-y^{2}}$-wave Pomeranchuk instability by ultrasound. Phys. Rev. B 80, 155123 (2009)

[All96] P. B. Allen et al.: Transport properties, thermodynamic properties, and electronic structure of SrRuO 3 . Phys. Rev. B 53, 4393-4398 (1996)

[And02] Y. Ando, K. Segawa, S. Komiya und A. N. Lavrov: Electrical Resistivity Anisotropy from Self-Organized One Dimensionality in High-Temperature Superconductors. Phys. Rev. Lett. 88, 137005 (2002)

[Aok98] Y. Aoki et al.: Thermal properties of metamagnetic transition in heavyfermion systems. J. Magn. Magn. Mater. 177-181, 271 -276 (1998)

[BCS57] J. Bardeen, L. N. Cooper und J. R. Schrieffer: Microscopic Theory of Superconductivity. Phys. Rev. 106, 162-164 (1957)

[BM86] J. G. Bednorz und K. A. Müller: Possible high- $T_{\mathrm{c}}$ superconductivity in the Ba-La-Cu-O system. Zeitschrift für Physik B: Condensed Matter 64, 189193 (1986)

[BP91] B. Baym und C. Pethick: Landau Fermi-liquid theory: concepts and applications. Wiley \& Sons (1991)

[BPM10] S. Bühler-Paschen und P. Mohn: Festkörperphysik II. Vorlesungsskript, TU Wien (2010/2011). URL: http://sxs . ifp . tuwien . ac . at/skripten/ (abgerufen Jan. 2011)

[Ber09] A. M. Berridge, A. G. Green, S. A. Grigera und B. D. Simons: Inhomogeneous Magnetic Phases: A Fulde-Ferrell-Larkin-Ovchinnikov-Like Phase in $\mathrm{Sr}_{3} \mathrm{Ru}_{2} \mathrm{O}_{7}$. Phys. Rev. Lett. 102, 136404 (2009) 
[Ber10] A. M. Berridge, S. A. Grigera, B. D. Simons und A. G. Green: Magnetic analog of the Fulde-Ferrell-Larkin-Ovchinnikov phase in $\mathrm{Sr}_{3} \mathrm{Ru}_{2} \mathrm{O}_{7}$. Phys. Rev. B 81, 054429 (2010)

[Blu01] S. Blundell: Magnetism in Condensed Matter. Oxford University Press (2001)

[Boo78] C. de Boor: A practical guide to splines. Springer, New York (1978)

[Bor07] R. A. Borzi et al.: Formation of a Nematic Fluid at High Fields in $\mathrm{Sr}_{3} R u_{2} \mathrm{O}_{7}$. Science 315, 214-217 (2007)

[Bro08] D. M. Broun: What lies beneath the dome? Nature Physics 4, 170$172(2008)$

[Chi02] M. Chiao et al.: Effect of pressure on metamagnetic $\mathrm{Sr}_{3} \mathrm{Ru}_{2} \mathrm{O}_{7}$. Physica B: Condensed Matter 312-313, 698 -699 (2002)

[Chu10] J.-H. Chu et al.: In-Plane Resistivity Anisotropy in an Underdoped Iron Arsenide Superconductor. Science 329, 824-826 (2010)

[Cop] Low Temperature Properties of Copper. URL: http://www.copper.org/ resources/properties/ (abgerufen März 2010)

[Dao10] R. Daou et al.: Broken rotational symmetry in the pseudogap phase of a high- $T_{\mathrm{c}}$ superconductor. Nature 463, 519 (2010)

[EH00] C. Enss und S. Hunklinger: Tieftemperaturphysik. Springer (2000)

[Flo02] J. Flouquet, P. Haen, S. Raymond, D. Aoki und G. Knebel: Itinerant metamagnetism of $\mathrm{CeRu}_{2} \mathrm{Si}_{2}$ : bringing out the dead. Comparison with the new $\mathrm{Sr}_{3} \mathrm{Ru}_{2} \mathrm{O}_{7}$ case. Physica B: Condensed Matter 319, 251 -261 (2002)

[Flo95] J. Flouquet et al.: The heavy fermion compound CeRu $\mathrm{Si}_{2}$ : Magnetic instability, lattice quasicollapse and metamagnetism. Physica B: Condensed Matter 215, $77-87$ (1995)

[Fra10] E. Fradkin, S. A. Kivelson, M. J. Lawler, J. P. Eisenstein und A. P. Mackenzie: Nematic Fermi Fluids in Condensed Matter Physics. Annual Review of Condensed Matter Physics 1, 153-178 (2010)

[GK80] W. Gebhardt und U. Krey: Phasenübergänge und kritische Phänomene. Vieweg Verlag (1980)

[GR05] M. Garst und A. Rosch: Sign change of the Grüneisen parameter and magnetocaloric effect near quantum critical points. Phys. Rev. B 72, 205129 (2005)

[GSS08] P. Gegenwart, Q. Si und F. Steglich: Quantum criticality in heavy-fermion metals. Nature Physics 4, 186-197 (2008)

[Gar03] M. Garst: Quantenphasenübergänge: Grüneisenparameter, dimensionaler Crossover und gekoppelte Störstellen. Dissertation, Universität Karlsruhe (2003) 
[Geg06a] P. Gegenwart, F. Weickert, R. Perry und Y. Maeno: Low-temperature magnetostriction of $\mathrm{Sr}_{3} \mathrm{Ru}_{2} \mathrm{O}_{7}$. Physica B: Condensed Matter 378-380, 117118 (2006)

[Geg06b] P. Gegenwart, F. Weickert, M. Garst, R. S. Perry und Y. Maeno: Metamagnetic Quantum Criticality in $\mathrm{Sr}_{3} \mathrm{Ru}_{2} \mathrm{O}_{7}$ studied by Thermal Expansion. Phys. Rev. Lett. 96, 136402 (2006)

[Geg07] P. Gegenwart et al.: Multiple energy scales at a quantum critical point. Science 315, 969 (2007)

[Gol92] N. Goldenfeld: Lectures on Phase Transitions and the Renormalization Group. Addison-Wesley (1992)

[Gri01] S. A. Grigera et al.: Magnetic Field-Tuned Quantum Criticality in the Metallic Ruthenate $\mathrm{Sr}_{3} \mathrm{Ru}_{2} \mathrm{O}_{7}$. Science 294, 329-332 (2001)

[Gri03] S. A. Grigera et al.: Angular dependence of the magnetic susceptibility in the itinerant metamagnet $\mathrm{Sr}_{3} \mathrm{Ru}_{2} \mathrm{O}_{7}$. Phys. Rev. B 67, 214427 (2003)

[Gri04] S. A. Grigera et al.: Disorder-Sensitive Phase Formation Linked to Metamagnetic Quantum Criticality. Science 306, 1154-1157 (2004)

[Gri70] R. B. Griffiths: Dependence of Critical Indices on a Parameter. Phys. Rev. Lett. 24, 1479-1482 (1970)

[Grü08] E. Grüneisen: Über die thermische Ausdehnung und die spezifische Wärme der Metalle. Annalen der Physik 331, 211-216 (1908)

[HM76] R. Hocken und M. R. Moldover: Ising Critical Exponents in Real Fluids: An Experiment. Phys. Rev. Lett. 37, 29-32 (1976)

[Hah70] T. A. Hahn: Thermal Expansion of Copper from 20 to $800 \mathrm{~K}$ - Standard Reference Material 736. Journal of Applied Physics 41, 5096-5101 (1970)

[Her76] J. A. Hertz: Quantum critical phenomena. Phys. Rev. B 14, 1165$1184(1976)$

[IH74] H. Ikeda und K. Hirakawa: Neutron scattering study of two-dimensional Ising nature of $\mathrm{K}_{2} \mathrm{CoF}_{4}$. Solid State Commun. 14, 529 -532 (1974)

[Ike00] S.-I. Ikeda, Y. Maeno, S. Nakatsuji, M. Kosaka und Y. Uwatoko: Ground state in $\mathrm{Sr}_{3} \mathrm{Ru}_{2} \mathrm{O}_{7}$ : Fermi liquid close to a ferromagnetic instability. Phys. Rev. B 62, R6089-R6092 (2000)

[Ike75] H. Ikeda, I. Hatta, A. Ikushima und K. Hirakawa: Observation of a Symmetric Logarithmic Singularity in the Specific Heat of $K_{2} C_{o} F_{4}$. J. Phys. Soc. Jpn. 39, 827-828 (1975)

[KDP80] K. v. Klitzing, G. Dorda und M. Pepper: New Method for High-Accuracy Determination of the Fine-Structure Constant Based on Quantized Hall Resistance. Phys. Rev. Lett. 45, 494-497 (1980) 
[KFS96] A. A. Koulakov, M. M. Fogler und B. I. Shklovskii: Charge Density Wave in Two-Dimensional Electron Liquid in Weak Magnetic Field. Phys. Rev. Lett. 76, 499-502 (1996)

[KO11] H. Kamerlingh-Onnes: Further Experiments with Liquid Helium. Comm. Leiden 120b, 122b, 124c (1911)

[Kal11] A. Kalz: Monte-Carlo-Simulation eines 2d-Ising-Modells in externen Magnetfeldern. Persönliche Mitteilung, Universität Göttingen, Institut für theoretische Physik (2011)

[Kiv11] S. A. Kivelson, Stanford University. Persönliche Mitteilung (2011)

[Kre09] C. Krellner et al.: Violation of Critical Universality at the Antiferromagnetic Phase Transition of $\mathrm{YbRh}_{2} \mathrm{Si}_{2}$. Phys. Rev. Lett. 102, 196402 (2009)

[Küc03] R. Küchler et al.: Divergence of the Grüneisen Ratio at Quantum Critical Points in Heavy Fermion Metals. Phys. Rev. Lett. 91, 066405 (2003)

[Küc05] R. Küchler: Thermische Ausdehnung und divergierendes Grüneisenverhaltnis in Schwere-Fermionen-Systemen. Dissertation, Technische Universität Dresden (2005)

[LEB70] J. A. Lipa, C. Edwards und M. J. Buckingham: Precision Measurement of the Specific Heat of $\mathrm{CO}_{2}$ near the Critical Point. Phys. Rev. Lett. 25, 1086-1090 (1970)

[LW10] W.-C. Lee und C. Wu. Microscopic Theory of the Thermodynamic Properties of $\mathrm{Sr}_{3} \mathrm{Ru}_{2} \mathrm{O}_{7}$. 2010. eprint: arXiv:1008.2486

[Lan57] L. D. Landau: The theory of a Fermi liquid. Sov. Phys. JETP 3, 920925 (1957)

[Lan91] M. Lang: Dissertation, Technische Universität Darmstadt (1991)

[Li199] M. P. Lilly, K. B. Cooper, J. P. Eisenstein, L. N. Pfeiffer und K. W. West: Evidence for an Anisotropic State of Two-Dimensional Electrons in High Landau Levels. Phys. Rev. Lett. 82, 394-397 (1999)

[Löh07] H. v. Löhneysen, A. Rosch, M. Vojta und P. Wölfle: Fermi-liquid instabilities at magnetic quantum phase transitions. Rev. Mod. Phys. 79, 10151075 (2007)

[MDN94] J. Mattsson, C. Djurberg und P. Nordblad: Determination of the critical exponent $\beta$ from measurements of a weak spontaneous magnetisation in the $3 d$ Ising antiferromagnet $\mathrm{FeF}_{2}$. J. Magn. Magn. Mater. 136, L23 L28 (1994)

[MI08] K. Momma und F. Izumi: VESTA: a three-dimensional visualization system for electronic and structural analysis. J. Appl. Crystallogr. 41, 653$658(2008)$ 
[MM03] A. P. Mackenzie und Y. Maeno: The superconductivity of $\mathrm{Sr}_{2} \mathrm{RuO}_{4}$ and the physics of spin-triplet pairing. Rev. Mod. Phys. 75, 657-712 (2003)

[MMB95] M. Marinelli, F. Mercuri und D. P. Belanger: Critical behaviour of thermal parameters of $\mathrm{FeF}_{2}$ at the Néel temperature. J. Magn. Magn. Mater. 140144, $1547-1548$ (1995)

[Mat98] N. D. Mathur et al.: Magnetically mediated superconductivity in heavy fermion compounds. Nature 394, 39-43 (1998)

[McC04] W. McComb: Renormalization Methods: A guide for beginners. Oxford University Press (2004)

[Mer09] J.-F. Mercure et al.: Quantum Oscillations in the Anomalous Phase in $\mathrm{Sr}_{3} \mathrm{Ru}_{2} \mathrm{O}_{7}$. Phys. Rev. Lett. 103, 176401 (2009)

[Mer10] J.-F. Mercure et al.: Quantum oscillations near the metamagnetic transition in $\mathrm{Sr}_{3} \mathrm{Ru}_{2} \mathrm{O}_{7}$. Phys. Rev. B 81, 235103 (2010)

[Mil02] A. J. Millis, A. J. Schofield, G. G. Lonzarich und S. A. Grigera: Metamagnetic Quantum Criticality in Metals. Phys. Rev. Lett. 88, 217204 (2002)

[Mil93] A. J. Millis: Effect of a nonzero temperature on quantum critical points in itinerant fermion systems. Phys. Rev. B 48, 7183-7196 (1993)

[Nag] NAG Library Function: nag_1d_spline_fit (). URL: http://www . nag . com/numeric/CL/nagdoc_cl09/xhtml/E02/e02bec.xml (abgerufen Jan. 2011)

[O'H00] R. C. O'Handley: Modern Magnetic Materials. Wiley \& Sons (2000)

[PG10] J. Paglione und R. Greene: High-temperature superconductivity in ironbased materials. Nature Physics 6, 645-658 (2010)

[PS83] R Pott und R Schefzyk: Apparatus for measuring the thermal expansion of solids between 1.5 and 380 K. J. Phys. E: Sci. Instrum. 16, 444-449 (1983)

[Per01] R. S. Perry et al.: Metamagnetism and Critical Fluctuations in High Quality Single Crystals of the Bilayer Ruthenate $\mathrm{Sr}_{3} \mathrm{Ru}_{2} \mathrm{O}_{7}$. Phys. Rev. Lett. 86, 2661-2664 (2001)

[Per04] R. S. Perry et al.: Multiple First-Order Metamagnetic Transitions and Quantum Oscillations in Ultrapure $\mathrm{Sr}_{3} \mathrm{Ru}_{2} \mathrm{O}_{7}$. Phys. Rev. Lett. 92, 166602 (2004)

[Pob07] F. Pobell: Matter and Methods at Low Temperatures. Springer (2007)

[Pom58] I. J. Pomeranchuk: Stability of a Fermi Liquid. JETP 35, 524 (1958)

[Pue88] L. Puech et al.: High-field magnetostriction in the pseudo-metamagnetic heavy-fermion system CeRu $\mathrm{Si}_{2}$. J. Low Temp. Phys. 70, 237-251 (1988)

[RM67] R. P. Reed und R. P. Mikesell: Low temperature mechanical properties of copper and selected copper alloys. National Bureau of Standards monograph, Band 101 (1967) 
[Rag09] S. Raghu et al.: Microscopic theory of the nematic phase in $\mathrm{Sr}_{3} \mathrm{Ru}_{2} \mathrm{O}_{7}$. Phys. Rev. B 79, 214402 (2009)

[Ros09] A. W. Rost, R. S. Perry, J.-F. Mercure, A. P. Mackenzie und S. A. Grigera: Entropy Landscape of Phase Formation Associated with Quantum Criticality in $\mathrm{Sr}_{3} \mathrm{Ru}_{2} \mathrm{O}_{7}$. Science 325, 1360-1363 (2009)

[Ros10] A. Rost et al.: Power law specific heat divergence in $\mathrm{Sr}_{3} \mathrm{Ru}_{2} \mathrm{O}_{7}$. Phys. Status Solidi B 247, 513-515 (2010)

[Set91] J. P. Sethna: Order Parameters, Broken Symmetry and Topology. Lectures in Complex Systems (1991)

[Sha00a] H. Shaked, J. D. Jorgensen, O. Chmaissem, S. Ikeda und Y. Maeno: Neutron Diffraction Study of the Structural Distortions in $\mathrm{Sr}_{3} \mathrm{Ru}_{2} \mathrm{O}_{7}$. Journal of Solid State Chemistry 154, 361 -367 (2000)

[Sha00b] H. Shaked et al.: Temperature and pressure effects on the crystal structure of $\mathrm{Sr}_{3} \mathrm{Ru}_{2} \mathrm{O}_{7}$ : Evidence for electronically driven structural responses. Phys. Rev. B 62, 8725-8730 (2000)

[Son97] S. L. Sondhi, S. M. Girvin, J. P. Carini und D. Shahar: Continuous quantum phase transitions. Rev. Mod. Phys. 69, 315-333 (1997)

[Ste79] F. Steglich et al.: Superconductivity in the Presence of Strong Pauli Paramagnetism: $\mathrm{CeCu}_{2} \mathrm{Si}_{2}$. Phys. Rev. Lett. 43, 1892-1896 (1979)

[TG11] Y. Tokiwa und P. Gegenwart: High-resolution alternating-field technique to determine the magnetocaloric effect of metals down to very low temperatures. Rev. Sci. Instr. 82, 013905 (2011)

[TSG82] D. C. Tsui, H. L. Stormer und A. C. Gossard: Two-Dimensional Magnetotransport in the Extreme Quantum Limit. Phys. Rev. Lett. 48, 1559$1562(1982)$

[Tam08] A. Tamai et al.: Fermi Surface and van Hove Singularities in the Itinerant Metamagnet $\mathrm{Sr}_{3} \mathrm{Ru}_{2} \mathrm{O}_{7}$. Phys. Rev. Lett. 101, 026407 (2008)

[Tok09] Y. Tokiwa, T. Radu, C. Geibel, F. Steglich und P. Gegenwart: Divergence of the Magnetic Grüneisen Ratio at the Field-Induced Quantum Critical Point in $\mathrm{YbRh}_{2} \mathrm{Si}_{2}$. Phys. Rev. Lett. 102, 066401 (2009)

[Voj03] M. Vojta: Quantum phase transitions. Reports on Progress in Physics 66, 2069 (2003)

[Wei10] F. Weickert, M. Brando, F. Steglich, P. Gegenwart und M. Garst: Universal signatures of the metamagnetic quantum critical endpoint: Application to $C e R u_{2} S i_{2}$. Phys. Rev. B 81, 134438 (2010)

[Wos07] J. Wosnitza: From Thermodynamically Driven Phase Transitions to Quantum Critical Phenomena. J. Low Temp. Phys. 147, 249-278 (2007)

[Wu10] C. Wu, University of California, San Diego. Persönliche Mitteilung (2010) 
[YPM06] H. Yaguchi, R. S. Perry und Y. Maeno: Ferromagnetism Induced by Uniaxial Pressure in the Itinerant Metamagnet $\mathrm{Sr}_{3} \mathrm{Ru}_{2} \mathrm{O}_{7}$. AIP Conf. Proc. 850, 1203-1204 (2006)

[Zhu03] L. Zhu, M. Garst, A. Rosch und Q. Si: Universally Diverging Grüneisen Parameter and the Magnetocaloric Effect Close to Quantum Critical Points. Phys. Rev. Lett. 91, 066404 (2003) 



\section{Danksagung}

Viele Menschen haben mit ihrer Hilfe und Unterstützung das Gelingen dieser Arbeit erst möglich gemacht. Sie sollen an dieser Stelle nicht unerwähnt bleiben:

Prof. Dr. Philipp Gegenwart hat diese Arbeit mit großem Engagement und Interesse begleitet. Ich danke ihm für die Möglichkeit, auf einem faszinierenden und anspruchsvollen Forschungsgebiet zu arbeiten, für seine stetige Diskussionsbereitschaft und für die Geduld und das Vertrauen, welche er mir während der Promotionszeit entgegengebrachte.

Prof. Dr. Michael Lang vom Physikalischen Institut der Goethe-Universität Frankfurt am Main danke ich für seine freundliche Bereitschaft, das Korreferat für diese Arbeit zu übernehmen.

Prof. Dr. Klaus Winzer stand mir in experimentellen Fragen stets mit Rat und Tat zur Seite und ließ mich in vielen Diskussionen an seiner reichhaltigen Erfahrung teilhaben. Als leidenschaftlicher Experimentalphysiker war er mir ein Vorbild.

Dr. Yoshifumi Tokiwa danke ich für die stets gute und erfreuliche Zusammenarbeit sowie für die Messungen der spezifischen Wärme. Mit Ratschlägen zur Tieftemperaturtechnik und vielfältigen Diskussionen hat er mir sehr geholfen.

MaIK Schubert hatte auch in frustrierenden Augenblicken immer einen aufmunternden Spruch auf Lager. Danke für die nette gemeinsame Zeit im Labor und für das gewissenhafte Lektorat dieser Arbeit!

MANuel Mchalwat danke ich für die angenehme Bürogemeinschaft, anregende Diskussionen über die Feinheiten des wissenschaftlichen Textsatzes sowie für weiteres Korrekturlesen.

Der gesamten Arbeitsgruppe Tieftemperaturphysik gilt mein Dank für eine angenehme und freundschaftliche Arbeitsatmosphäre, nette Gruppenabende und leckeres indisches und japanisches Essen. 
Hartmut Eichenberg, Elmar Gatzemeier und die weiteren Mitarbeiter der Feinmechanischen Werkstatt des I. Physikalischen Instituts haben die Arbeiten stets mit großer Hilfsbereitschaft unterstützt und erkennen in Kapitel 3 sicherlich das eine oder andere von ihnen gefertigte Werkstück wieder. Besonders dankbar bin ich für die Möglichkeit, im Rahmen eines kleinen Praktikums die Grundlagen der Materialbearbeitung zu erlernen. Auch den MitarbeiterInnen der Zentralwerkstatt der Fakultät für Physik sowie der Elektronikwerkstatt im Institut für Materialphysik bin ich zu Dank verpflichtet.

Kathrin Gehrke und Carsten Mahn danke ich für vielfältige technische und UTA FILIPPICH für moralische Unterstützung.

Unverzichtbar für die Tieftemperaturexperimente waren MAnfred Piepenbring und später Bastian BEYLAND, die sich mit großem Einsatz um die Heliumverflüssigung gekümmert haben. Für die gute Zusammenarbeit herzlichen Dank!

ANSGAR KALZ vom Institut für theoretische Physik danke ich für die Simulationen zum zweidimensionalen Isingmodell sowie für Diskussionen, in denen er mir die Theorie auf meinem Niveau nahegebracht hat.

Auch den hier nicht namentlich erwähnten Kolleginnen und Kollegen im I. Physikalischen Institut möchte ich meinen Dank aussprechen. Sie alle haben meine Jahre im Institut zu einer schönen Zeit gemacht, an die ich gern zurückdenke.

Zum Schluß möchte ich besonders meinen Eltern und Geschwistern für ihre große Unterstützung in jeder Hinsicht danken. Meiner Freundin Lisa danke ich für die schönen gemeinsamen letzten Jahre. 




\section{Lebenslauf}

\section{Christian Matthias Stingl}

geb. am 14.03.1981 in Lemgo

\begin{aligned} & \hline 2000 Abitur \\ & \hline Okt. 2001 $\begin{array}{l}\text { Studium der Physik (Nebenfach: Informatik) an der Universi- } \\ \text { tät Göttingen }\end{array} \\ &$\hline Juni 2003 Vordiplom \\ & \hline Okt. 2005 $\begin{array}{l}\text { Diplomarbeit im I. Physikalischen Institut, Arbeitsgruppe } \\ \text { Prof. Samwer. Thema: „Physikalische Eigenschaften und Mi- } \\ \text { krostruktur von dünnen } \mathrm{La}_{067} \mathrm{Ce}_{033} \mathrm{MnO}_{3} \text {-Schichten“ }\end{array} \\ &$\hline Jan. 2007 Diplom \\ & \hline seit April 2007 wissenschaftlicher Mitarbeiter im I. Physikalischen Institut, Ar- \\ & beitsgruppe Prof. Gegenwart \\ & \hline Mai 2011 Disputationsprüfung \\ & \hline\end{aligned}

